\title{
Wohnquartiere und Kriminalität Überblick über die Forschung zu den sozialräumlichen Dimensionen urbaner Kriminalität
}

\section{Dietrich Oberwittler}

M ax-Planck-Institut für ausländisches und internationales Strafrecht, Abt. Kriminologie, Freiburg

erscheint in:

Oberwittler, Dietrich / Rabold, Susanne / Baier, Dirk (Hg.) (2013):

Städtische Armutsquartiere - Kriminelle Lebenswelten? Studien zu sozialräumlichen Kontexteffekten auf Jugendkriminalität und Krimi- nalitätswahrnehmungen, Wiesbaden: Springer VS, S. 4595

1. Auflage 2013, Print ISBN 978-3-531-16976-7, Online ISBN 978-3-531-93244-6 DOI: 10.1007/978-3-531-93244-6_2

d.oberwittler@mpicc.de https://www.mpicc.de/de/home/oberwittler.html 


\section{Wohnquartiere und Kriminalität - Überblick über die Forschung zu den sozialräumlichen Dimensionen urbaner Kriminalität}

Dietrich Oberwittler

Auch wenn der Cyberspace eine zunehmende Bedeutung für bekannte und neuartige Formen der Kriminalität erlangt, bleiben viele herkömmliche Formen strafbaren Verhaltens - z.B. Sachbeschädigung, Diebstahl, Wohnungseinbruch, Raub, Körperverletzung - doch fest in Raum und Zeit verankert. Erhebliche Teile des Phänomens Kriminalität werden daher auch in Zukunft ihre sozialräumliche Dimension behalten, die die Kriminalitätsforschung von Beginn beschäftigt hat. Seit der Industrialisierung und Urbanisierung im 19. Jahrhundert gelten Großstädte und vor allem großstädtische Armutsquartiere als besonders mit Kriminalität belastete Räume, während zuvor einige Kriminalitätsformen im Gegenteil häufiger in ländlichen Gebieten registriert worden waren (Eisner 1997; Thome 2002). Dass Kriminalität und andere Formen der Unordnung (disorder oder incivilities), die häufig mit Kriminalität in Verbindung gebracht werden, innerhalb der Städte sehr ungleich verteilt sind, hat Konsequenzen für die lebensweltlichen Erfahrungen und Einschätzungen der betroffenen Menschen, entscheidet mit über die Attraktivität und Lebensqualität von Wohngebieten und ist ein Ausgangspunkt für diverse polizeiliche, kommunalpolitische und städtebauliche Strategien.

Die Forschung zu räumlichen Aspekten von Kriminalität hat in den letzten fünfzehn Jahren enorm zugenommen und ist mittlerweile nur noch schwer zu überblicken (Bottoms 2007, 2012; Kubrin 2009; Sampson 2006; Sampson et al. 2002; Taylor 2010; Wikström 2007). Neben der Forschung zu Stadtvierteleffekten auf Jugenddelinquenz, die in diesem Beitrag im Mittelpunkt stehen soll, und die ihren Bezugspunkt immer noch in der klassischen Chicago School der 1930er und 1940er Jahre hat (Shaw/McKay 1969 [1942]), haben sich Studien zu den räumlichen Verteilungsmustern der Tatorte von Kriminalität zu eigenständigen Forschungszweigen entwickelt. Während städtische Räume bzw. Wohngebiete in der erstgenannten Perspektive hauptsächlich als Entwicklungs- und Handlungskontexte für ihre (jugendlichen) Bewohner gesehen werden, geht es in der zweiten Perspektive um städtische Räume als Kontexte für Ereignisse (Wikström/Sampson 2003). Auch in dieser Perspektive spielt der Zusammenhang zwischen sozialräumlichen Benachteiligungen und Kriminalität eine wichtige, aber nicht mehr die einzig entscheidende Rolle. Kriminalitätsereignisse können sich zwischen 
den lokalen Bewohnern abspielen (als Täter, Opfer oder sonstige Anwesende), müssen es jedoch nicht. Denn städtische Räume sind eine „Bühne“ für soziale Interaktionen zwischen Menschen unterschiedlicher Herkunft und Rollen: An-

wohner, Besucher, Arbeitende, Einkau- fende, usw. Dies gilt besonders für zentrumsnahe Räume, die teils sehr hohe Kriminalitätsbe- lastungen und „,hot spots“ aufweisen (Braga 2005; Sherman et al. 1989). Ein eigenes Forschungsfeld (crime pattern theory) untersucht die räumliche Verteilung krimineller Ereignisse als Ergebnis von Mobilitätsmustern von Tätern und Opfern (Brantingham/Brantingham 2008). Ein benachbartes Forschungsfeld analysiert Tatortverteilungen vorrangig unter den Prämissen der Rational Choice-Theorie und geographischer Muster günstiger Tatgelegenheiten, auch um daraus situative Präventionsmaßnahmen abzuleiten (z.B. Bernasco 2010; Bowers/Johnson 2005; Clarke 2009; Cornish/Clarke 1986). Zu den Faktoren, die Tatgelegenheiten beeinflussen, gehört jedoch auch die von der Chicago School ins Zentrum gerückte informelle Sozialkontrolle und der soziale Zusammenhalt der Bewohner - Gegenstand eines weiteren Untersuchungsfeldes -, so dass es durchaus Überlappungen zwischen diesen Perspektiven gibt. Einen recht umfassenden theoretischen Rahmen für diese unterschiedlichen Perspektiven auf räumliche Kriminalitätsverteilungen bietet das sogenannte „systemic model of crime“ (Bursik 1999, Bursik/Grasmick 1993; Warner/Rountree 1997), das unter anderem auf Überlegungen der Stadtsoziologen Kasarda und Janowitz (1974) zum sozialen Zusammenhalt von Großstadtbewohnern zurückgeht.

Allen genannten Forschungsperspektiven ist gemein, dass sie städtischen Räumen eine eigenständige kausale Bedeutung - unabhängig von der Rolle der Individuen, die sich in ihnen aufhalten - für die Genese von Kriminalität zugestehen. Es wird angenommen, dass die geographischen Raumeinheiten kollektive Merkmale haben, die sich entweder aus der sozialen $\mathrm{Zu}$ sammensetzung und den wechselseitigen Beziehungen der Menschen, die sich in ihnen aufhalten, entwickeln, oder sich aus physischen und städtebaulichen Eigenschaften, der geographischen Lage, der Verkehrswege usw. ergeben. Die Vorstellung der „Emergenz“ kollektiver Eigenschaften aus einer Menge individueller Handlungen ist ein Kernbestandteil des MakroMikro-Makro-Modells nach Coleman und Boudon und entspricht dem „Mechanismus [oder der Logik] der Aggregation“ (Hedström/Swedberg 1998; vgl. Boudon 1998; Esser 1996; Friedrichs 1988; Greve/Schnabel/Schützeichel 2008). Wenn diese kollektiven Eigenschaften 
der Makro-Ebene wiederum Einfluss auf das individuelle Verhalten von Menschen, die sich in diesen Kontexten aufhalten, haben, dann entspricht dies im Makro-Mikro-Makro-Modell dem „Mechanismus [oder der Logik] der Situation“. Da Kriminalität aus der Perspektive geographischer Einheiten stets aus der Summe vieler einzelner Handlungen besteht, stellen Makro-Mikro-Makro-Verbindungen notwendige Elemente in sozialräumlichen Kriminalitätstheorien dar.

\section{Das ,,systemische Model““ der Kriminalität - wechselseitige Beziehungen zwischen struk- tureller Benachteiligung, sozialem Kapital und Kriminalität auf der Ebene von Stadt- vierteln}

Die Rolle von sozialem Kapital und kollektiver Wirksamkeit bei der Kontrolle von Kriminalität

Im „systemischen Model“ wird der kollektiven sozialen Organisation eines Wohnquartiers vor allem der Fähigkeit, für die Einhaltung von Verhaltensnormen zu sorgen - eine kausale Bedeutung für das Ausmaß der lokalen Kriminalität zugeschrieben, aber auch umgekehrt nach den Rückwirkungen von Kriminalität und Unordnung auf diese soziale Organisation gefragt. Soziale Bindungen und Vertrauen gelten als Voraussetzungen für die Bereitschaft der Bewohner, sich für das gemeinsame Wohl einzusetzen, und die Summe dieser individuellen Handlungsdispositionen bildet ein kollektives soziales Kapital, von dem alle profitieren können in der Sprache der Ökonomie eine ,ppositive Externalität““(Coleman 1988: 116; Granovetter 1973: 1373; vgl. Friedrichs/Oberwittler 2007).

Eine grundlegende Annahme des Models ist, dass die soziale Organisation von Wohnquartieren sehr stark von strukturellen Merkmalen beeinflusst wird und damit einen Mechanismus bildet, welcher den empirisch immer wieder bestätigten Zusammenhang zwischen struktureller Benachteiligung und Kriminalität erklären kann (Pratt/Cullen 2005). Konzentrierte Armut und andere strukturelle Benachteiligungen verursachen nach dieser Vorstellung Kriminalität nicht direkt, sondern vermittelt über eine unzureichende kollektive Organisation der Bewohner (,, indirect effect hypothesis“, Bursik/Grasmick 1993). Dies wird in der schematischen Darstellung des „systemischen Modells“ (Abbildung 1) dadurch angezeigt, dass der hauptsächliche Wirkungspfad von „,konzentrierter Benachteiligung“ zunächst zur „,sozialen Organi- 
sation“ und von dort weiter zu „Kriminalität“ geht. Tatsächlich zeigt sich jedoch in vielen empirischen Studien, dass trotz Berücksichtigung der sozialen Organisation ein direkter Einfluss struktureller Benachteiligung auf Kriminalität bestehen bleibt (z.B. Sampson/Raudenbush/Earls 1997).

Bis hierher entspricht diese Argumentation der bis heute einflussreichen Theorie der sozialen Desorganisation von Shaw/McKay (1969 [1942]). Diese stellten statistische Zusammenhänge der Jugendkriminalitätsraten in den Chicagoer Stadtvierteln mit drei Merkmalen sozialräumlicher Benachteiligung fest (Armut, ethnische Heterogenität und residentielle Instabilität) und erklärten dies unter anderem mit der Schwierigkeit der Bewohner, sich zum kooperativen Handeln zusammenzufinden (Shaw/McKay 1969 [1942], 184). Allerdings dauerte es bis in die 1990er Jahre, bis die Existenz dieser vermittelnden sozialen Prozesse mit Hilfe von Befragungsdaten empirisch bestätigt wurde. Bellair (1997) konnte in einer Aggregatanalyse von Struktur- und Befragungsdaten in 60 Wohngebieten zeigen, dass der Einfluss struktureller Probleme auf Kriminalität in der Tat teilweise durch soziale Bindungen im Wohngebiet vermittelt wird. Diese und andere Studien zeigten jedoch auch, dass sehr enge Bindungen und intensive Interaktionen zwischen den Bewoh-

nern weniger nützlich für die Kontrolle von Krimi- nalität sind als schwache Bindungen (Hipp/Perrin 2006; vgl. Granovetter 1973), ja dass dichte Netzwerke und Interaktionen sogar kriminalitätsfördernd wirken können, wenn sie an deviante Subkulturen gekoppelt sind (Bellair/Browning 2010; Browning/Feinberg/Dietz 2004; Warner/Rountree 1997).

*** Abb. 1 ungefähr hier

Im Konzept der „,kollektiven Wirksamkeit“ (collective efficacy), der von Robert Sampson (2004, 2006, 2012) weiterentwickelten Version der Theorie der sozialen Desorganisation, wird daher weniger Wert auf soziale Netzwerke und Interaktionen als auf generalisiertes Vertrauen und Verhaltenserwartungen im kollektiven Interesse gelegt. Die Kernidee der kollektiven Wirksamkeit ist, dass sich Bewohner dann erfolgreich für das gemeinsame Wohl der 
Nachbarschaft einsetzen - konkret indem sie bei Fehlverhalten von Kindern und Jugendlichen einschreiten -, wenn eine Basis gegenseitigen Vertrauens und geteilter Normen vorhanden ist. Dafür ist es nach Sampson (2006: 40) in der modernen, urbanisierten Lebenswelt nicht erforderlich „, that my neighbor or local police officer be my friend“. Ebenso trugen die Erkenntnisse neuerer ethnographischer Studien (Carr 2003) dazu bei, dass im Konzept der kollektiven Wirksamkeit auch das Einschalten der Polizei unter den Begriff der informellen Sozialkontrolle subsumiert wird, solange die Initiative dazu von den Bewohnern ausgeht und es sich nicht um eine pro-aktive Polizeistrategie handelt (Sampson 2006: 13). Entsprech-

end lautet die Frage im Erhebungsinstrument zur Messung der kollektiven Wirksamkeit schlicht, ob die Nachbarn ,etwas tun würden“, wenn Jugendliche z.B. Grafittis sprühen (Sampson et al. 1997, vgl. Friedrichs/Oberwittler 2007 und Lüdemann 2006b für deutsche Versionen der Skala, vgl. auch Peter/Schlepper/Lüdemann in diesem Band).

Das Konzept der kollektiven Wirksamkeit wurde erstmals 1995 in der Bewohnerbefragung im Rahmen des „,Project on Human Development in Chicago Neighborhoods“ empirisch überprüft. Dabei wurden 8.782 Bewohner in 343 „,neighorhood clusters “, zusammengefasste Volkszählungsbezirke (census tracts) mit durchschnittlich ca. 8.000 Einwohnern in der Stadt Chicago befragt. Das zentrale Ergebnis war, dass die kollektive Wirksamkeit wie angenommen einen wesentlichen Teil des Effekts der struktureller Benachteiligung auf die Häufigkeit von Gewaltdelikten in den Wohnquartieren vermittelte, wobei jedoch noch ein direkter Wirkungspfad der konzentrierten Armut erhalten blieb. Die sozialräumliche Verteilung der Gewaltkriminalität in Chicago konnte durch die Berücksichtigung der kollektiven Wirksamkeit signifikant besser aufgeklärt werden als durch die strukturellen Merkmale alleine. Inzwischen haben weitere Studien das Konzept der kollektiven Wirksamkeit angewendet und die Chicagoer Ergebnisse bestätigt, so z.B. in Schweden (Sampson/Wikström 2008), den Niederlanden (Nieuwbeerta et al.2008), Großbritannien (Wikström et al. 2012) und Australien (Mazerolle et al. 2010). In einer deutschen Studie hatte die kollektive Wirksamkeit dagegen keinen signifikanten Effekt auf Gewaltkriminalität über den Einfluss struktureller Benachteiligungen hinaus (Friedrichs/Oberwittler 2007).

In allen empirischen Studien zeigte sich, dass die Unterschiede zwischen Wohnquartieren im Ausmaß der kollektiven Wirksamkeit zu zwei Dritteln oder mehr durch die Konzentration struktureller Benachteiligungen, vor allem gemessen durch Armut, aber auch durch residenti- 
elle Instabilität erklärt werden konnten. Einige europäische Studien bestätigten darüber hinaus die umstrittene These Putnams (2007), dass ethnische Heterogenität mit einem Vertrauensverlust der Bewohner einhergeht (Andrews 2009; Laurence 2011; Oberwittler/Wikström 2011; Portes/Vickstrom 2011; Wikström et al. 2012: 182), der unter anderem vermutlich auf seltenere Kontakte zwischen Bewohnern unterschiedlicher ethnischer Herkunft zurückzuführen ist (Stolle/Soroka/Johnston 2008). Denn auch wenn soziale Interaktionen und freundschaftliche Bindungen zwischen Bewohnern kein Bestandteil des Konzepts der kollektiven Wirksamkeit sind, so tragen sie doch indirekt durch die Förderung der allgemeinen Zufriedenheit mit dem Wohnquartier und des Vertrauens in die Nachbarn zur Stärkung des kollektiven Sozialkapitals bei (Burchfield 2009; Friedrichs/Oberwittler 2007; Gibson et al. 2002; Silver/Miller 2004).

\section{Rückwirkungen von Kriminalität und Unordnung auf Sozialkapital und Sozialstruktur}

Ein sehr wichtiger Teil des systemischen Modells befasst sich mit den Rückwirkungen oder feed back-Prozessen von Kriminalität und verschiedenen Formen der Unordnung im öffentlichen Raum, die meist unter den Begriffen incivilities oder disorder zusammengefasst werden, auf verschiedene Aspekte der sozialen Organisation von Wohngebieten. Der Grundge-

danke ist, dass persönliche Erfahrungen und Beobachtungen von abweichendem Verhalten (oder auch Berichte darüber) Kriminalitätsfurcht fördern, Vertrauen zerstören, zu sozialem Rückzug führen und die Bereitschaft zum Handeln im gemeinsamen Interesse reduzieren könnten.

Diese Rückwirkungen sind in Abbildung 1 als graue Pfeile von „Kriminalität/Unordnung“ zu „,sozialer Rückzug“ und von dort zu „,soziale Organisation“ dargestellt. Schon um kausale Wirkungen des Sozialkapitals auf Kriminalität abzusichern und dabei das Endogenitätsproblem auszuschalten, welches zu einer Überschätzung von statistischen Zusammenhängen führen kann (Sobel 1995), sollten diese Feedback-Prozesse in empirischen Modellen berücksichtigt werden. Aus diesem Grund haben Sampson/Raudenbush/Earls (1997) zeitlich vorhergehende Kriminalitätsdaten als Kontrollvariable in ihr Modell aufgenommen und gezeigt, dass Gewalt im Zeitverlauf zu einer Reduzierung der kollektiven Wirksamkeit führte. Über ähnli- 
che Rückwirkungen berichtete Bellair (2000): Gewaltkriminalität führte zu einem Rückgang der informellen Sozialkontrolle, hingegen hatte Einbruchsdiebstahl einen gegenteiligen Effekt. Auch Lüdemann (2006b, vgl. Peter/Schlepper/Lüdemann in diesem Band) fand einen positiven Effekt von indirekten Viktimisierungen (und von wahrgenommer Unordnung, s.u.) auf die praktizierte Sozialkontrolle der Bewohner.

Es waren vor allem zwei Studien bzw. Erklärungsansätze, die die Beziehung zwischen urbaner Unordnung und Unsicherheitswahrnehmungen in Hinblick auf die soziale Entwicklung von Wohnquartieren in den Mittelpunkt der Aufmerksamkeit gerückt und eine Flut von weiteren Studien und Veröffentlichungen ausgelöst haben: Wesley Skogan argumentierte in seinem Buch Disorder and Decline (1990, vgl. Skogan 2008), dass benachteiligte Wohnquartiere der amerikanischen Großstädte durch Kriminalität und Unordnung in eine Abwärtsspirale des Verfalls hineingezogen werden, und Wilson und Kelling postulierten in ihrem berühmt gewordenen Aufsatz Broken Windows (1982) einen Mechanismus, demzufolge alltägliche Phä nomene physischer Unordnung wie Müll und zerbrochene Fensterscheiben als sichtbarer Ausdruck einer mangelnden Sozialkontrolle im öffentlichen Raum potenzielle Täter zur Kriminalität animieren und die Bewohner weiter verunsichern. Diese Hypothesen wurden in einer Vielzahl von Studien empirischen Tests unterzogen. In einer aufwändigen Längsschnittstudie mit Daten des British Crime Surveys fanden Markowitz et al. (2001) Hinweise auf einen solchen negativen Feedback-Kreislauf zwischen Unordnung, Angst und Vertrauen: „Cohesion decreases disorder, which increases fear, which decreases cohesion” (Markowitz et al. 2001: 310). Weitere Studien haben die Annahme negativer Rückwirkungen von Kriminalität und Unordnung bestätigt (Brunton-Smith 2011; Brunton-Smith/Sturgis 2011; Gault/Silver 2008; Garcia/Taylor/Lawton. 2007; Pauwels/Hardyns/Van den Velde 2010; Ross/Jang 1999; Steenbeck/Hipp 2011; Taylor et al. 2011, vgl. Häfele in diesem Band). Es ist jedoch umstritten, ob Unordnung einen von Kriminalität abgrenzbaren Platz und eine eigenständige kausale Rolle im systemischen Modell haben sollte (Gau/Pratt 2008; Raudenbush/Sampson 1999b; Sampson 2009; Xu/Fielder/Flaming 2005). Eine der methodisch anspruchsvollsten Längsschnittstudien, die unter der Leitung von Ralph Taylor in Baltimore durchgeführt wurde, ist in Schlussfolgerungen vorsichtiger. Diese Studie fand keinen signifikanten Effekt von Unordnung auf eine spätere Zunahme von Kriminalitätsfurcht oder auf eine abnehmende

Zufriedenheit mit dem Wohnquartier (Robinson et al. 2003), obwohl der Zusammenhang zwischen Unordnung und Kriminalitätsfurcht im Querschnitt sehr eng war, wie auch in vielen 
anderen Studien (Hir- tenlehner 2008; Lüdemann 2006a; Oberwittler 2008; Wyant 2008, vgl. Häfele in diesem Band). Taylor und seine Kollegen argumentieren, dass diese starke Korrelation zwischen der Wahrnehmung von Unordnung und Kriminalitätsfurcht Ausdruck einer Überschneidung der Bedeutungsinhalte der Fragen ist und deswegen erheblich überschätzt wird: Befragte, die sehr ängstlich sind, nehmen auch mehr Unordnung war (Covington/Taylor 1991; Perkins/Taylor 1996; Taylor 2001: 228, zu methodischen Aspekten s.u.). Sowohl Kriminalitätsfurcht als auch Wahrnehmungen von Unordnung sind schwer fassbare Konzepte, die stark von subjektiv unterschiedlichen Empfindsamkeiten beeinflusst werden und zudem diffuse, allgemeinere gesellschaftliche Sorgen ausdrücken (Covington/Taylor 1991; Farrall/Jackson/Gray 2009; Hirtenlehner 2006; Hummelsheim et al. 2011; Innes 2004; Jackson 2004).

Aus diesem Grunde wurden in einigen Studien eigene systematische Beobachtungen physischer und sozialer Zeichen der Unordnung durchgeführt, zusätzlich oder an Stelle der Bewohnerbefragungen. Im Project of Human Development in Chicago Neighborhoods lagen die Korrelationen zwischen befragungsbasierten subjektiven Wahrnehmungen und beobachtungsbasierten Messungen der physischen und sozialen Disorder-Phänomene zwischen $\mathrm{r}=.65$ und 70 (Sampson/Raudenbush 1999: 31). Anhand derselben Daten demonstrierten Sampson und Raudenbush (2004), dass die subjektiven Wahrnehmungen von Unordnung von der sozialen und ethnischen Zusammensetzung der Wohnquartiere mitbestimmt wird: Relativ zu den systematischen Beobachtungen nehmen Bewohner von sozial benachteiligten und ethnisch segregierten Wohnquartieren mehr Unordnung wahr, und zwar unabhängig von ihrem eigenen sozialen und ethischen Status. Eifler/Thume/Schnell (2009) zeigten mit den Daten deutscher Opferbefragungen (die allerdings keine Kontextdaten der Wohnquartiere enthielten), dass eine erhöhte Sensibilität für Unordnung mit eigenen oder von Bekannten berichteten Viktimisierungen erklärbar ist (vgl. auch Häfele in diesem Band).

Das Schema des systemischen Modells (Abbildung 1) enthält einen zweiten und größeren Rückkopplungs-Zyklus, nämlich den der Verschärfung konzentrierter Benachteiligungen durch selektive Abwanderung aus den von Kriminalität und Unordnung betroffenen Wohnquartieren. Ziehen diejenigen, die es sich leisten können, aus den problematischen Quartieren weg, so verändert sich die sozialstrukturelle Zusammensetzung weiter zum Schlechteren. Für diesen Prozess gibt es einige empirische Belege wiederum aus den USA, wo die Problemlage zweifelsohne weitaus schärfer ist als in europäischen Großstädten (Cullen/Levitt 1999; South/Messner 2000, vgl. Massey 1996). Morenoff und Sampson (1997) zeigten, dass die ethnische Segregation in Chicago zwischen 1970 und 1990 unter anderem wegen der räumlichen Verteilung der Tötungsdelikte zugenommen hat: Stadtviertel mit einer hohen Mordrate verloren insgesamt an Einwohnern, jedoch ging nur die weiße Bevölkerung stark zurück, während 
die absolute Anzahl schwarzer Einwohner sogar anstieg. Möglicherweise werden diese negativen Effekte von Kriminalität auf die demographische Entwicklung von Stadtvierteln noch unterschätzt, da es Hinweise darauf gibt, dass in den besonders benachteiligten Wohnquartieren weniger Straftaten angezeigt und polizeilich

registriert werden, so dass die offiziellen Kri- minalitätsraten zu einer Unterschätzung der Problemlagen führen (Goudriaan/Wittebrood/Nieuwbeerta 2006; Kirk/Matsuda 2011, siehe Tab. 1; Tita/Petras/Greenbaum 2006). Es gibt demgegenüber keine empirischen Bestätigungen der aus dem Labeling Approach abgelei- teten Annahme, dass eine negative soziale Entwicklung von Wohnquartieren lediglich durch eine kollektive Stigmatisierung aufgrund selektiven Polizeihandelns ausgelöst werde (vgl.

Belina/Wehrheim 2011).

Kriminalität und Unordnung im öffentlichen Raum sind allerdings nicht die einzigen Faktoren, die über die Attraktivität von Wohnquartieren entscheiden und für die Wohnsitzwahl relevant sind; die ethnische Zusammensetzung der Bewohner und die wahrgenommene Qualität der lokalen Schulen sind weitere, miteinander verbundene Faktoren (Baur/Häußermann 2009; Farwick 2012; Häußermann 2008). Schließlich sollte auch nicht übersehen werden, dass es trotz empirischer Bestätigungen des „Teufelskreises“ aus sozialen Benachteiligungen, mangelndem Sozialkapital und Kriminalität auch Beispiele für entgegengesetzte Prozesse gibt, wie z.B. die Gentrifizierung. Dieser Prozess betrifft vor allem innenstadtnahe Wohnquartiere, die aufgrund ihrer Lage, ihrer Bausubstanz und eines allgemeinen Nachfragetrends nach zentrumsnaher Wohnlagen wieder für sozial bessergestellte Schichten attraktiv sind und durch deren Zuzug ,aufgewertet“ werden - mit nicht unproblematischen Auswirkungen hinsichtlich der Mietpreisentwicklung und Verdrängung sozial schwacher Bewohner (Holm 2010, 2012). Die Gentrifizierung demonstriert, dass es gegenläufige sozialräumliche Wandlungsprozesse gibt, die offensichtlich wesentlich stärker sind als die zuvor beschriebene Abwärtsdynamik. Dabei darf der makro-gesellschaftliche Kontext nicht übersehen werden, zu dem die ökonomische Entwicklung ebenso dazu gehört wie auch ein seit Mitte der 1990er Jahre stark fallender Trend bei vielen Formen der Kriminalität wie Wohnungseinbruch und KfZ-Diebstahl (Oberwittler 2012). Über die Mechanismen der Gentrifizierung in Hinblick auf die lokale Kriminalitätsentwicklung, z.B. ob ein Rückgang der Kriminalität Voraussetzung oder Folge der Gentrifizierung ist, ist recht wenig bekannt (Kirk/Laub 2010). 


\title{
Sozialräumliche Kontexteffekte auf Jugenddelinquenz
}

\author{
Theoretische Erklärungsansätze
}

Führt die Konzentration sozialer Benachteiligungen in Wohnquartieren zu einer Verstärkung von problematischen Verhaltensweisen und Entwicklungstendenzen von Kindern und Jugendlichen (im Folgenden vereinfacht ,Jugendliche“), die in diesen Quartieren wohnen? Dies ist ganz kurz gefasst die Kernfrage, die hinter der Hypothese sozialräumlicher Kontexteffekte auf Jugendliche steht. Ging es im vorhergehenden Abschnitt zum „systemischen Modell“ um die Wirkungen der kollektiven sozialen Organisation auf die aggregierte Summe von Kriminalitätsereignissen in Wohnquartieren (hinter denen letztlich auch einzelne Ereignisse stehen), so liegt der Fokus - wie bei der klassischen Chicago School der 1930er

Jahre - im Folgenden auf den Wirkungen auf das individuelle abweichende Verhalten und die Entwicklung von Jugendlichen.

Auswirkungen sozialräumlicher Konzentrationen von Benachteiligungen - in erster Linie sind damit materielle und Bildungsarmut sowie Migrationsstatus gemeint - werden nicht nur in Hinblick auf Delinquenz und Kriminalität, sondern auf eine Vielzahl von Verhaltensbereichen diskutiert (vgl. allgemein Sampson/Morenoff/Gannon-Rowley 2002): Bildungserfolg (Schulleistungen, Schulabbruch) (z.B. Ditton 2009; Harding et al. 2011), Gesundheit (psychisches Wohlbefinden, Ernährungsverhalten/Gewicht) (Diez Roux/Mair 2010; Reijneveld et al. 2005), Sexualverhalten (z.B. Browning et al. 2008, siehe Tab. 1; Harding 2007; Lupton/Kneale 2012) und Substanzkonsum (z.B. Fauth/Roth/Brooks-Gunn 2007; Maimon/Browning 2012). Viele dieser Verhaltensbereiche weisen Querverbindungen und Wechselwirkungen auf und werden daher häufig als Aspekte eines übergeordneten „Risiko-“ oder „Problemverhaltens“ von Jugendlichen aufgefasst und in einigen Studien auch gemeinsam untersucht (Harding 2010). Insbesondere unterscheiden sich die theoretischen Erklärungsansätze in Hinblick auf sozialräumliche Kontexteffekte zwischen diesen Bereichen nur wenig.

Für die Erklärung von Kontexteffekten auf abweichendes Verhalten von Jugendlichen ist die Theorie der sozialen Desorganisation von Shaw und McKay (1969 [1942]) bis heute der zent- 
rale Ausgangspunkt geblieben (vgl. Kunadt, Rabold/Baier in diesem Band). Dies liegt auch daran, dass Shaw und McKay mehrere Wirkungsmechanismen der sozialräumlichen Strukturbedingungen auf Kinder und Jugendliche postuliert haben: Einerseits den Effekt der informellen Sozialkontrolle der (erwachsenen) Bewohner über Jugendliche, der im „,systemischen Modell“" und im Ansatz der kollektiven Wirksamkeit die entscheidende Rolle spielt (s.o.), andererseits aber auch den Effekt der Kontakte zwischen delinquenten Jugendlichen, den sie sich als einen Prozess der „cultural transmission“ devianter Normen und Verhaltensmuster von einer Generation von Jugendlichen auf die jeweils nachwachsende Generation vorstellten (Shaw und McKay 1969 [1942], 168). Dieser Zweig der Desorganisationstheorie, der an die klassische Lerntheorie (Sutherland/Cressey 1960) anknüpfte und später in den Subkulturtheorien seine Fortsetzung fand (Cohen 1955; Miller 1958), wurde jedoch später unter dem Einfluss von Travis Hirschi (1969, vgl. Kornhauser 1978) über Jahrzehnte hinweg unterbewertet und ist erst seit einigen Jahren wieder stärker in den Vordergrund gerückt (Akers/Jensen 2006; Harding 2008 (siehe Tab. 1), 2009b; Haynie/Osgood 2005; Warr 2002; vgl. Kubrin 2009, vgl. Windzio in diesem Band). Der Mechanismus der sozialräumlichen Verstärkung von Jugenddelinquenz durch Gleichaltrige ist mit Krankheitsepidemien verglichen worden: Wenn subkulturelle Einstellungen in einem sozial segregierten Wohnquartier durch die Konzentration benachteiligter Familien Fuß fassen, könnte der Kontakt zwischen den Jugendlichen deviante Normen verbreiten und verstärken (Crane 1991). Die Anwesenheit vieler Jugendlicher mit delinquenten Neigungen stellt auch einen situativen Faktor für Delinquenz dar, denn Jugendliche begehen Delikte ganz überwiegend in Gemeinschaft mit anderen (Wikström et al. 2012, 329). Jugendgangs als eine mehr oder weniger organisierte Form von Jugenddelinquenz haben meist einen starken territorialen Bezug und bewirken bei ihren Mitgliedern eine deutliche Intensivierung ihrer Delinquenz (Klein/Maxson 2006; Klein/Weerman/Thornberry 2006; Pitt 2008; Thornberry et al. 2003).

Diese beiden Wirkungspfade - informelle Sozialkontrolle durch die Erwachsenen einerseits, delinquente Einflüsse vorrangig durch Gleichaltrige andererseits - wurden, da sie unterschiedlichen Theorietraditionen zuzuordnen sind, häufig als konkurrierend wahrgenommen und bislang sehr selten gemeinsam untersucht. Angemessener erscheint es jedoch, diese Aspekte der sozialen Organisation von Wohnquartieren in einem wechselseitigen Zusammenhang zu sehen, wie dies James Coleman bereits in den 1960er Jahren getan hat, als er den Desorganisationsansatz auf die neu entstehenden Suburbs der amerikanischen Mittelschicht bezog. Je 
schwächer der Zusammenhalt und die kollektive Wirksamkeit der Erwachsenen, desto besser kann sich dadurch nach Coleman die (subkulturelle) Organisation der Jugendlichen entwickeln: „One result of the highly developed adolescent community and minimal adult community is a relative powerlessness of adults to control their children. [...] Thus there is a proliferation of community among the children, as community among parents desintegrates" (Coleman 1966, 705). Später hat Coleman (1988) diesen Gedanken zum Konzept der „intergenerational closure" als einer Form des kollektiven Sozialkapitals weiterentwickelt. Einen weiteren Wirkungsmechanismus hat William J. Wilson (1987; 1997, vgl. Friedrichs/Galster/Musterd 2003, vgl. Rabold/Baier in diesem Band) in seinen sehr einflussreichen Büchern über die Verschärfung der Armut in den afro-amerikanischen Ghettos der US-Großstädte im Zuge der De-Industrialisierung beschrieben: Je seltener reguläre Arbeitsverhältnisse der erwachsenen Ghetto-Bewohner werden, desto mehr fehlen positive Rollenvorbilder für Jugendliche (und werden durch deviante Vorbilder ersetzt). Auf der individuellen Ebene könnte dieser Effekt sowohl anomietheoretisch, durch Frustrationen angesichts blockierter sozialer Chancen (Agnew 1999), als auch subkulturtheoretisch erklärt werden.

Erweiterungen des Theoriemodells werden erforderlich, wenn man berücksichtigt, dass Schulen einen weiteren und geographisch häufig nicht mit dem Wohnquartier deckungsgleichen Kontext darstellen, der für die Sozialisation von Jugendlichen zweifelsohne sehr relevant ist (Kauppinen 2008; Kirk 2009; Oberwittler 2007a, 2007b; vgl. Pauwels in diesem Band; zu methodischen Aspekten s.u.). Nicht anders als bei Wohnquartieren auch werden in Bezug auf Kontexteffekte von Schulen unterschiedliche Wirkungsmechanismen angenommen, nämlich in erster Linie Effekte der Schulorganisation, für die Schulleitungen und Lehrkräfte verantwortlich sind (Bradshaw/Sawyer/O`Brennan 2009; Payne/Gottfredson/Gottfredson 2003), als auch Effekte der Beeinflussung durch Mitschüler (Fuchs/Schmalz 2010; Harris/Duncan/ Boisjoly 2002, Lo et al. 2011).

Eine weitere wichtige Ausdifferenzierung der Erklärungsansätze betrifft die zeitliche Dimension: Kinder und Jugendliche durchlaufen verschiedene Entwicklungsphasen, in denen der Charakter und die Intensität der Umwelterfahrungen sehr unterschiedlich sind und die Bedeutung der Eltern als Vermittler von und „Beschützer“ vor schädlichen sozialräumlichen Einflüssen tendenziell abnimmt (Ingolsby et al. 2006). Wikström/Sampson (2003) haben eine Unterscheidung zwischen kurzfristigen und langfristigen Effekten des Wohnquartiers vorgeschlagen: Während kurzfristige Einflüsse vor allem die aktuellen Gelegenheitsstrukturen für 
durch die langfris- tigen Einflüsse die Einstellungen und Neigungen zur Delinquenz geprägt (,ecological context of development“).

*** Abbildung 2 ungefähr hier

Abbildung 2 fasst wesentliche Aspekte dieser theoretischen Überlegungen in einem Schema zusammen. Das Individuum befindet sich hier in einem Koordinatensystem, das sowohl die räumliche als auch die zeitliche Dimension abbildet. Der Terminologie Bronfenbrenners (1979) folgend wird das Individuum selbst und seine unmittelbare familiäre Umwelt als ökologische Mikro-Ebene, die schulische und kleinräumliche Umwelt als Meso-Ebene und der entfernte regionale und nationale Kontext als Makro-Ebene bezeichnet. In der frühen Kindheit ist die Bedeutung der Familie für die Gestaltung der sozialen Umwelt noch sehr groß; Einflüsse der Meso-Ebene auf das Individuum werden kaum direkt wirksam, sondern eher durch die familiäre Situation vermittelt. Dies ändert sich in dem Maße, wie das Kind direkte soziale Interaktionen mit der Umwelt außerhalb der eigenen Familie aufnimmt. Im Jugendalter ist dementsprechend die familiäre „Schutzhülle“ um das Individuum dünner und der direkte Interaktionspfeil zwischen Individuum und Meso-Ebene stärker als in der frühen Kindheit. Zudem differenzieren sich die sozialökologischen Kontexte der Meso-Ebene mit der Erweiterung der Aktionsräume des Jugendlichen aus. Stadtviertel- und Schulkontext als die beiden wichtigsten Kontexte überlappen sich zwar häufig, sind jedoch nicht identisch. In der zeitlichen Perspektive übt die Meso-Ebene (ebenso wie die Makro-Ebene, deren Effekte

hier nicht dargestellt sind) nicht nur unmittelbare, sondern auch verzögerte und kumulative Wirkungen

aus, indem sie längerfristig stabile Einstellungen und Motivationen prägen kann. Der beidseitig gerichtete Pfeil deutet Interaktionen zwischen den Individuum und der sozialräumlichen 
Kontexte an, d.h. die individuellen Jugendlichen sind nicht nur passive Empfänger von Kontexteinflüssen, sondern setzen sich im Sinne des Modells des ,,produktiv realitätsverarbeitenden Subjekts“ (Hurrelmann 1983, vgl. Magnusson/Stattin 2006) aktiv mit ihrer sozialen Umwelt auseinander. Wenn man diesen Gedanken ernst nimmt, dann liegt auch die Vermutung nahe, dass Jugendliche individuell sehr unterschiedlich auf sozialräumliche Kontextbedingungen reagieren (siehe unten). Aber erst seit einigen Jahren verfolgen Forscher diese Perspektive vermehrt und untersuchen, warum Jugendliche mehr oder weniger (oder gar nicht) empfänglich für Kontexteinflüsse sind, und welche individuellen Faktoren für differentielle Wirkungen des Kontextes verantwortlich sind. In der ersten, weitaus längeren Phase der Forschungsgeschichte wurde die Frage nach sozialräumlichen Kontexteffekten auf Jugendliche in der Regel so verstanden, dass es um durchschnittliche Effekte auf „die“ Jugendlichen in den betroffenen Wohnquartieren ging. Ich werde diese beiden Perspektiven entlang der historischen Forschungsentwicklung ebenfalls in dieser Reihenfolge behandeln.

Empirische Resultate zur Bedeutung von sozialräumlichen Kontexten für Kinder und Jugendliche - „globale“ Effekte

Shaw und McKay selbst konnten die Existenz sozialräumlicher Kontexteffekte auf Jugendliche aufgrund der damaligen methodischen Beschränkungen nicht empirisch überprüfen. Anhand aggregierter offizieller Daten ließ sich lediglich zeigen, dass bestimmte Wohnquartiere Chicagos trotz ständigen Bevölkerungsaustausches über lange Zeiträume hohe Konzentrationen von jugendlichen Straftätern aufwiesen. Als sich in den 1960er und 1970er Jahren Jugendbefragungen zur selbstberichteten Delinquenz als Methode der Kriminalsoziologie mehr und mehr durchsetzen, standen sozialräumliche Fragestellungen zunächst nicht im Vordergrund (Kivivuori 2011; Krohn et al. 2010). Bei den damals noch seltenen Studien zu Kontexteffekten dominierte die Vorstellung, dass sich in Wohnquartieren mit der steigenden Konzentration sozial benachteiligter Familien eine deviante Subkultur ausbreitet und im Extremfall zur „Leitkultur“ wird, der sich Jugendliche kaum entziehen können. In einer der ersten Studien auf der Basis von Selbstberichtsdaten stellten Clarke und Wenninger (1962, 833) fest „, that there are community-wide norms which are related to illegal behavior and to which juveniles adhere regardless of their social class origin “. Detlev Frehsee, der in den 1970er Jahren die erste vergleichbare Studie in Deutschland durchführte, formulierte gleichlautend: „Innerhalb der Wohnbereiche findet also eine kulturelle Anpassung an das dominante Normensystem statt“ (Frehsee 1979: 349). Inzwischen wurde diese Vorstellung einer homogenen de- 
vianten Subkultur zugunsten der Idee einer Normenheterogenität aufgegeben (die sich allerdings auch schon in der Studie von Shaw und McKay (1969 [1942], 182) findet), welche die Jugendlichen vor die Notwendigkeit stellt, sich zwischen unter-

schiedlichen Werten und Hand- lungsalternativen entscheiden zu müssen (Anderson 1999; Friedrichs 1997; Harding 2007, 2010, s.u.).

Die meisten Studien zu sozialräumlichen Kontexteffekten auf Jugenddelinquenz wurden und werden in den USA durchgeführt. Hier sind vor allem drei sehr aufwändige Längsschnittstudien zu nennen, deren Design optimal auf die Analyse von Kontexteinflüssen ausgerichtet ist. Das „Project on Human Development in Chicago Neighborhoods“ (PHDCN) wurde von Mitte der 1990er Jahre bis in die frühen 2000er Jahre durchgeführt und schloss drei Befragungswellen von sieben Alterskohorten mit Interviews der Kinder und Jugendlichen sowie ihrer Eltern ein. Zusätzlich wurden eine Bewohnerbefragung sowie systematische Beobachtungen in Wohnquartieren durchgeführt, ergänzt durch offizielle Zensus-, Polizei- und sonstige Daten. Die „National Longitudinal Study of Adolescent Health“ („Add Health“) ist eine national-repräsentative Studie, die ebenfalls Mitte der 1990er Jahre mit einer Stichprobe von rund 90.000 Jugendlichen begann und über viele Wellen bis heute weitergeführt wird. Sie umfasst neben Befragungen auch schulweite Netzwerkanalysen und medizinische Untersuchungen einschließlich Gentests. Den individuellen Befragungsdaten können die Volkszählungsdaten der Wohnquartiere (census tracts) als Kontextmerkmale zugespielt werden. Um die Dimension dieser Studie deutlich zu machen, genügt der Hinweis, dass bis heute beinahe 4.000 Veröffentlichungen mit „Add Health“-Daten entstanden sind. Die „Moving to Opportunity“ (MTO)-Studie schließlich ist durch ihren experimentellen Charakter einzigartig: Zufällig ausgewählte Familien, die in extrem armen Wohnquartieren leben, können in ein „,besseres“ Wohnquartier umziehen, und die soziale Entwicklung der Jugendlichen wird über Jahre beobachtet (De Luca/Dayton 2009; Kling/Ludwig/Katz 2005; Ludwig et al. 2008; siehe Tab. 1, vgl. Burdick-Will/Ludwig in diesem Band).

Diese und weitere Studien in den USA haben klare empirische Hinweise auf die Existenz sozialräumlicher Kontexteffekte auf Jugenddelinquenz und andere Formen von Problemverhalten erbracht. Alle Studien stimmen darin überein, dass die Stärke der Kontexteffekte - gemessen an den Varianzanteilen in multivariaten Erklärungsmodellen - weit hinter der Stärke der 
individuellen Einflussfaktoren zurücksteht (Elliott etal. 2006: 276). Tabelle 1 bietet eine strukturierte Übersicht über einige wichtige Veröffentlichungen aus den USA und aus europäischen Ländern, die den internationalen Forschungsstand zu sozialräumlichen Kontexteffekten auf Jugenddelinquenz abbilden können. Einige der Veröffentlichungen befassen sich mit verwandten Verhaltensbereichen wie Gesundheit und Bildungserfolg, und die meisten Veröffentlichungen basieren auf Mehrebenenanalysen von Befragungsdaten, die nach Wohnquartieren oder Schulen (oder beiden) gruppiert wurden. Es ist jedoch wichtig zu betonen, dass die Auswahl der Studien weder systematisch noch vollständig ist.

*** Tabelle 1 ungefähr hier (Seitenformat QUER!!)

In der Chicagoer Studie erklärten Kontexteffekte des Wohnquartiers 60\% der höheren Gewaltbelastung der afro-amerikanischen gegenüber den weißen Jugendlichen (Sampson/Morenoff/Raudenbush 2005, vgl. Kirk 2008, 2009; siehe Tab. 1). Der in den USA bei der Erklärung von Gewalt oft im Vordergrund stehende ,race“-Faktor entpuppt sich demnach zumindest teilweise als eine Folge

sozialräumlicher Benachteiligungen. Auf der Basis derselben Chicagoer Daten zeigte sich in einer anderen Auswertung eine Verdoppelung der Wahrscheinlichkeit, schwere Gewalt auszuüben, wenn die Jugendlichen bis zu zwei Jahren zuvor Zeuge von Gewalt mit Schusswaffen geworden waren (Bingenheimer/Brennan/Earls 2005; vgl. Brookmeyer/Fanti/Henrich 2006; Harding 2009a; Patchin et al. 2006; siehe Tab. 1).Die Autoren interpretieren diesen statistisch gut abgesicherten Zusammenhang als kausalen Effekt des Wohnquartiers und sehen ihn als Beleg für die Verstärkungseffekte einer Gewalt-Subkultur. Auch die „Add Health“-Studie kann die Existenz von sozialräumlichen Verstärkungseffekten belegen. Die Armutskonzentration des Wohngebietes hat einen signifikanten Effekt auf die Gewaltdelinquenz der Jugendlichen und führt gemeinsam mit einer Reihe weiterer Risikofaktoren dazu, dass ethnische Zuordnungen statistisch gänzlich unbedeutend werden (Bellair/McNulty 2005, siehe Tab. 1). Auch De Coster/Heimer/Wittrock (2006: 741, siehe Tab. 1) kommen anhand der „Add Health“-Daten zu dem Schluss ,,that the relationship between violence and individual level, race, ethnicity, poverty, parents' education, and female headship 
can be explained in part by the types of communities in which families and individuals reside”. Andere Auswertungen mit den Daten der Chicagoer Studie und der „Add Health“-Studie belegen sozialräumliche Effekte auf Schulleistungen und Sexualverhalten (Browning/Leventhal/Brooks-Gunn 2005, Browning et al. 2008; Harding 2009a; Sampson/Sharkey/Raudenbush 2008, siehe Tab. 1). Ein zentrales und überraschendes Ergebnis der experimentellen MTO-Studie war, dass Mädchen positiver auf den Umzug reagierten als Jungen (Kling/Ludwig/Katz 2005; siehe Tab. 1, vgl. Burdick-Will/Ludwig in diesem Band).

Die europäische Forschung zu sozialräumlichen Wirkungen auf Jugenddelinquenz ist viel weniger entwickelt und methodisch weniger aufwändig. Es dominieren noch Querschnittsstudien, deren Ergebnisse zurückhaltender interpretiert werden müssen (siehe unten). Studien, die die Wirkungen konzentrierter Benachteiligungen in Wohngebieten untersuchen, haben keine eindeutigen Ergebnisse erbracht. So zeigten sich in Rotterdam (Rovers 1997), Antwerpen (Pauwels 2007, siehe Tab. 1), Peterborough (Wikström/Butterworth 2006, siehe Tab. 1) und Duisburg (Kunadt 2011, vgl. Kunadt in diesem Band) keine Kontexteffekte auf Jugenddelinquenz, während eine in den Jahren 1999 und 2000 in Köln und Freiburg durchgeführte Studie des Freiburger Max-Planck-Instituts für Strafrecht Hinweise auf recht starke Kontexteffekte erbrachte, die jedoch nur für einige Gruppen gelten (Oberwittler 2004a, 2004b, 2007b). Dieses hier als „Kölner/Freiburger Studie“ bezeichnete Forschungsprojekt stellte den in Deutschland bis dahin aufwändigsten Versuch dar, Nachbarschaftseffekte auf Jugenddelinquenz zu finden. Für einheimische Jugendliche mit auf das eigene Wohnquartier konzentriertem Freundeskreis verdoppelt sich nach den Ergebnissen der Kölner/Freiburger Studie beinahe die Wahrscheinlichkeit der schweren Delinquenz mit der Zunahme der sozialräumlichen Armut unter Kontrolle individueller Faktoren; gleichzeitig verliert der individuelle Arbeitslosen- bzw. Sozialhilfestatus der Eltern seine Signifikanz (Oberwittler 2004b). Bei Mädchen nimmt insbesondere die Gewaltneigung stark zu, bei Jungen eher die Wahrscheinlichkeit schwerer Eigentumsdelikte. Ebenso wie in den US-amerikanischen Studien wirkt die sozialstrukturelle Benachteiligung also eher kollektiv über die Wohngebiete als individuell auf jugendliche Delinquenz. Allerdings gilt dies erwartungswidrig nicht für Jugendliche aus Migrantenfami-

lien, für die sich keine sozialräumlichen Verstärkungseffekte fanden (Oberwittler 2004b, 2007b). Auch in einer national-repräsentativen Studie in den Niederlanden zeigte sich ein 
deutlicher Anstieg des Risikos psycho-sozialen Problemverhaltens von Kindern in den am stärksten benachteiligten Wohnquartieren (Reijneveld et al. 2005). In Bezug auf den Bildungserfolg von Jugendlichen in Schweden hat Brännström $(2004,2006)$ auf der Basis von Daten aus den 1960er Jahren gar keinen und auf der Basis von aktuelleren Daten (Brännström 2008, siehe Tabelle 1) schwache Kontexteffekte der Wohnquartiere gefunden, die jedoch hinter denen der Schule zurückstanden.

Schulen als Kontexte spielen naturgemäß vor allem bei Studien zum Bildungserfolg und zu abweichendem Verhalten in der Schule eine entscheidende Rolle. Eine der bislang wenigen deutschen Studien, die den Einfluss der sozialen Zusammensetzung des Schulkontextes (der allerdings als Proxy-Variable des Wohnquartiers interpretiert wird) auf Schulleistungen untersuchen, kommt zu dem Ergebnis, dass nicht die Kontexte mit der höchsten Konzentration sozialer Benachteiligungen die Schulleistungen verringern, sondern im Gegenteil die besonders „günstigen“ Kontexte, in denen gar keine oder sehr wenige Familien sozial benachteiligt sind, die Schulleistungen zusätzlich positiv beeinflussen (Helbig 2010, siehe Tab. 1; vgl. Ditton/Krüsken 2006). Hierbei handelt es sich um einen nichtlinearen, am oberen Ende der Verteilung stärker werdenden Kontexteffekt, der ähnlich auch von Sampson/Morenoff/Earls (1999) in Hinblick auf das kindbezogene kollektive Sozialkapital berichtet wurde.

Einige Studien konnten die Annahme bestätigen, dass die Schulorganisation und das „Schulklima“ einen eigenständigen Einfluss auf das Ausmaß des delinquenten Verhaltens der Schüler innerhalb, teils aber auch außerhalb der Schule haben (Brookmeyer/Fanti/Henrich 2006, siehe Tab. 1; vgl. Fuchs/Schmalz 2010; Gottfredson et al. 2005; Payne 2008). Eine Studie zur Entwicklung von Grundschulkindern fand heraus, dass das Ausmaß an Selbstkontrolle - einem wichtigen psychologischen Prädiktor von Jugenddelinquenz - negativ von dem Fehlverhalten der anderen Schüler in der Klasse beeinflusst wird, was als ein „Ansteckungseffekt“ interpretiert werden könnte (Beaver/Wright/Maume 2008; vgl. Pratt/Turner/Piquero 2004). Die bisherige empirische Forschung lässt sich so zusammenfassen, dass viele Studien Hinweise auf die Existenz sozialräumlicher Kontexteffekten auf Jugendliche gefunden haben, und zwar in den USA sehr viel deutlicher (und zudem methodisch besser abgesichert) als in Europa. Angesichts der wesentlich schärferen sozialen Ungleichheit, der Ghettoisierung von benachteiligten Bevölkerungsgruppen und dem niedrigeren Niveau des Wohlfahrtsstaates in den USA ist dieses Ergebnis auch nicht überraschend, sondern spricht eher für den Erfolg des stärker wohlfahrtsstaatlich orientierten europäischen Gesellschaftsmodells (vgl. Baier/Rabold 2010). Zudem zeigt sich, dass Schulen einen eigenständigen, anscheinend häufig auch wichtigeren sozialräumlichen Kontext darstellen, der nicht unter den Kontext des Wohnquartiers subsumiert werden kann. Schließlich deutet sich in einigen Studien bereits an, ohne dass sie explizit darauf ausgerichtet sind, dass nicht alle Jugendlichen, die in einem Wohnquartier le- 
ben, in gleicher Weise auf ungünstige sozialräumliche Einflüsse reagieren. Diese Erkenntnis ist fundamental und hat zu erheblichen Differenzie-

rungen in der Erforschung sozialräumlicher Kontexteffekte geführt, die Gegenstand des folgenden Abschnittes sind.

Empirische Resultate zur Bedeutung von sozialräumlichen Kontexten für Kinder und Jugendliche - differentielle Effekte

In den letzten Jahren hat sich das Interesse der Forschung mehr und mehr zu differentiellen Wirkungen sozialräumlicher Kontexte und deren Wechselwirkungen mit individuellen Merkmalen der Bewohner verlagert. Denn es macht wenig Sinn, von einem gleichmäßigen oder „durchschnittlichen“Effekt des Kontextes auf alle Jugendliche auszugehen, wenn bereits Merkmale wie Geschlecht oder Ethnie offenbar Wechselwirkungen mit den sozialräumlichen Merkmalen eingehen. Diese Beobachtung lässt sich zu der grundlegenden Frage ausbauen, ob und warum Jugendliche mit bestimmten individuellen oder Gruppenmerkmalen unterschiedlich auf Umwelteinflüsse reagieren. Wenn viele Jugendliche trotz Aufwachsens in benachteiligten Wohnquartieren keine Anzeichen von delinquentem Verhalten zeigen und eine erfolgreiche schulische und soziale Entwicklung durchmachen, liegt es nahe, nach Schutzfaktoren zu suchen, die negative sozialräumliche Einflüsse „abpuffern“ und zur Resilienz (Unempfindlichkeit) beitragen können (Elliott et al. 2006; vgl. allg. Luthar 2003). Denn diese Erkenntnisse könnten auch für die Prävention von jugendlichem Problemverhalten in benachteiligten Wohnquartieren hilfreich sein. Andererseits formuliert die so genannte ,amplification hypothesis" die Annahme, dass das gemeinsame Auftreten von individuellen und kontextuellen Benachteiligungen zu einer besonderen Verstärkung von Verhaltensrisiken führen kann (Hay et al. 2007)

Dies ist zunächst eine Herausforderung an die Theoriebildung, die meistens isoliert verfolgten Perspektiven auf individuelle Risikofaktoren einerseits und soziale Rahmenbedingungen des Verhaltens andererseits stärker miteinander zu verbinden. Das gelingt am ehesten mithilfe von integrativen Theorien, die sich bei der Erklärung abweichenden Verhaltens nicht auf einen 
einzigen Theorieansatz beschränken, wie z.B. die Lern- oder Kontrolltheorie, sondern verschiedene Aspekte so miteinander verknüpfen, dass deren Einflüsse und Wechselwirkungen in sinnvoller Weise abgebildet werden können (Oberwittler 2012, 827). Eine wichtige Anforderung dabei ist, die Vielzahl einzelner Einflussfaktoren so in einem Modell anzuordnen, dass proximale (nahe am Ergebnis liegende) und distale (weiter vom Ergebnis entfernt liegenden) Faktoren voneinander unterschieden und so indirekte Wirkungsmechanismen dargestellt werden können. Zum Beispiel kann die sozialstrukturelle Zusammensetzung des Wohnquartiers als ein distaler Einflussfaktor aufgefasst werden, der indirekt, durch vermittelnde Prozesse z.B. dem Anteil delinquenter Freunde und der devianten Orientierung - auf die Delinquenz von Jugendlichen wirkt. Das „Biopsychosocial Model“ der Entwicklungspsychologen Kenneth Dodge und Gregory Pettit (2003) ist ein Beispiel für ein solches integratives Theoriemodell, in dessen Zentrum kognitive und emotionale Wahrnehmungsmuster stehen, die aggressives Verhalten wahrscheinlicher machen. Dodge und Pettit nehmen an, dass abweichendes Verhalten von Jugendlichen vor allem dann entsteht, wenn

ungünstige individuelle Veranlagungen und ungünstige soziale Erfahrungen und Kontexteinflüsse aufeinandertreffen. Günstige individuelle Veranlagungen können sich hingegen als Schutzfaktoren gegen ungünstige Kontexteinflüsse auswirken und abweichendes Verhalten verhindern. Damit rücken Wechsel- wirkungen zwischen individuellen und kontextuellen Einflussfaktoren in den Mittelpunkt des Interesses, die in empirischen Studien als Interaktionseffekte modelliert werden können

(Oberwittler/Gerstner im Druck).

Eine neue, speziell auf die Analyse sozialräumlicher Kontexte zugeschnittene Theorie ist die „Situational Action Theory“ (SAT) von Per-Olof Wikström (2006, 2010; Wikström et al. 2012). Ausgehend von der Erkenntnis, dass das Wohnumfeld von Jugendlichen nicht notwendigerweise deren relevanter Verhaltenskontext ist, geht diese Theorie von den konkreten raum-zeitlichen Kontexten aus, in denen sich Jugendliche aufhalten, und in denen sie sich delinquent verhalten. Die SAT rückt den individuellen „Wahrnehmungs-Entscheidungs-Prozess“" für oder gegen den Normbruch ins Zentrum und fragt nach den Wechselwirkungen zwischen den Situationen, die sich durch unterschiedliche Gelegenheiten, Provokationen und Kontrollintensitäten auszeichnen, und den individuellen Neigungen für abweichendes Verhalten, die vor allem durch moralische Überzeugungen und exekutive Funktionen geprägt wer- 
den (Wikström/Treiber 2007). Das Modell rechnet sowohl mit bedeutenden individuellen Unterschieden in den Wahrnehmungen von Situationen und Handlungsentscheidungen als auch mit Unterschieden im kriminogenen Gehalt der räumlichen „Settings“, in denen sich Kriminalität als Ergebnis der Wechselwirkung von Person und Umwelt ereignet. Dieses Zusammentreffen von Personen und Settings bildet nach der SAT die unmittelbaren Ursachen von Kriminalität, während Einflüsse, die zu der Ausprägung von individuellen Verhaltenstendenzen und zur Gestaltung der Settings führen, als „causes of the causes “ eine vorgelagerte und indirekte Kausalwirkung haben. Damit werden in diesem Theoriemodell Elemente von Rational Choice-Theorien und des Routine Acitivities Approach mit „,klassischen“ täterorientierten Erklärungsansätzen verbunden, so dass potenziell alle Einflüsse, die einerseits auf die individuellen Verhaltensdispositionen und andererseits auf die Situationen wirken, in das Erklärungsmodell ,eingebaut“ werden können. In empirischen Analysen von Daten der Längsschnittstudie PADS+ (einer Stichprobe von etwa 700 Jugendlichen in Peterborough/Großbritannien) bestätigten sich die theoretischen Annahmen der SAT insofern, als dass delinquentes Verhalten dann am wahrscheinlichsten ist, wenn sich Jugendliche mit delinquenten Verhaltensdispositionen an Orten aufhalten, die sowohl Gelegenheiten als auch einen Mangel an kollektiver Kontrolle aufweisen, und dass weder dieselben Jugendlichen in anderen sozialräumlichen Kontexten noch andere Jugendliche in denselben sozialräumlichen Kontexten besonders delinquent handeln (Wikström et al. 2010, 2012).

$\mathrm{Zu}$ den Dimensionen individueller Unterschiede zwischen Jugendlichen, die in der Forschung in Hinblick auf die Empfänglichkeit oder Resilienz gegenüber ungünstigen sozialräumlichen Einflüssen untersucht wurden, gehören Persönlichkeitsmerkmale und neuropsychologische Risikofaktoren, Geschlecht, Familie und elterliches Erziehungsverhalten sowie

die (davon nicht unabhängigen) Beziehungen zu Gleichaltrigen. Im Folgenden sollen jeweils einige For- schungsergebnisse zu diesen Dimensionen knapp referiert werden.

\section{Persönlichkeitsmerkmale und neuropsychologische Risikofaktoren}

Empirische Forschungen zu Wechselwirkungen zwischen neuropsychologischen Risikofaktoren und sozialräumlichen Kontexten stecken noch in den Kinderschuhen. Gerade weil hier aus 
sozialwissenschaftlicher Perspektive sensible Fragen berührt und Ursachenfaktoren aus weit entfernt gelegenen Feldern miteinander in Beziehung gesetzt werden, ist eine besondere methodische Sorgfalt eine Grundvoraussetzung für valide Ergebnisse, die jedoch leider nicht immer erfüllt wird. Als Beispiel hierfür kann die Studie von Simons et al. (2011) dienen, die Wechselwirkungen zwischen genetisch bedingten Variationen in der Regulierung von Neurotransmittern (v.a. Serotonin und Dopamin) und widrigen Einflüssen des „social environment “ in Hinblick auf aggressives Verhalten untersuchen. Diese Studie gehört zu dem wachsenden Forschungsgebiet der Gen-Umwelt-Interaktionen (,G x E“) (Caspi et al. 2002; Guo/Roettger/Cai 2008; Rutter/Silberg 2002). Gen-Umwelt-Interaktionen bedeuten zum Beispiel, dass es von Umwelteinflüssen abhängig ist, ob sich bestimmte genetische Dispositionen auf aggressives Verhalten auswirken oder nicht. Die Messung dieses „social environments “ bei Simons et al. (2011: 893) ist jedoch nicht auf Eigenschaften des sozialräumlichen Kontextes ausgerichtet; vielmehr handelt es sich um eine zusammengefasste Skala, die so unterschiedliche Dinge wie „, supportive parenting “, „harsh parenting “, ,, school involvement“, „religious participation “, „, racial discrimination“, neighbourhood victimization, und „violent peers“ umfasst und damit keinesfalls als Kontextvariable interpretiert werden darf.

Demgegenüber operationalisieren Beaver et al. (2012) die soziale Umwelt in ihrer Analyse der „Add Health“-Daten korrekt mit den Zensusdaten der Wohngebiete der untersuchten Jugendlichen. Ihre Analyse ergibt, dass ein genetischer Polymorphismus der Dopamin-Rezeptoren nur in den $25 \%$ am meisten benachteiligten Wohngebieten einen signifikanten Effekt auf die Gewaltdelinquenz der Jugendlichen hat, nicht hingegen in den übrigen Wohngebieten. Dies würde bedeuten, dass ein genetisch bedingter Risikofaktor für aggressives Verhalten nur in sozialräumlichen Kontexten zum Tragen kommt, die durch konzentrierte Benachteiligungen (und in dieser Studie allerdings nicht gemessene Merkmale der sozialen Organisation wie niedriges Sozialkapital oder gewaltaffine Subkultur) gekennzeichnet sind. Andere Studien haben sich auf die Wechselwirkungen von sozialräumlichen Kontexten und niedriger Impulskontrolle konzentriert, die als ein starker Prädiktor für Delinquenz und als ein relativ stabiles Persönlichkeitsmerkmal gilt. Mit Daten der Pittsburgh Youth Study berechneten Lynam et al. (2000), dass sich eine hohe Impulsivität besonders stark in den am stärksten sozial benachteiligten Wohngebieten mit sozialem Wohnungsbau auf die Gewaltdelinquenz der männlichen Jugendlichen auswirkte. Über gleichlautende Ergebnisse berichteten Meier et al. 2008, während Beyers et al. (2001) und Wikström/Loeber (2000) im Gegenteil zu dem Schluss kamen, dass neuropsychologische Risikofaktoren vor allem bei Jugendlichen in sozial nicht benachteiligten Wohngebieten ursächlich für 
schwere Delinquenz sind, während in benachteiligten Wohngebieten eher soziale Faktoren als Ursachen für Delinquenz relevant sind.

\section{Familiäre Faktoren}

Familiäre Faktoren, insbesondere die Eltern-Kind-Beziehung und das elterliche Erziehungsverhalten haben unbestritten eine zentrale Bedeutung für die Genese von Jugenddelinquenz (Loeber/Farrington 1998; Schulz et al. 2011). Eine Reihe von Studien deuten darauf hin, dass ein positives Familienklima und Erziehungsverhalten als Schutz und „Puffer“ gegen ungünstige sozialräumliche Kontexteinflüsse wirken können. Daraus folgt umgekehrt, dass die Existenz familiärer Risikofaktoren unter ungünstigen Kontextbedingungen zu einer Verschärfung der Delinquenz führen kann. Jugendliche aus defizitären Familien in benachteiligten Wohngebieten sind demnach als besondere Risikogruppe anzusehen. Dies ist besonders für Alleinerziehende relevant, die aus wirtschaftlichen Gründen häufig gezwungen sind, in Wohnquartieren mit den niedrigsten Mieten zu wohnen, und gleichzeitig weniger Zeit und Ressourcen zur Erziehung haben als vollständige Familien. Hay et al. (2007, vgl. Wheaton/Clarke 2003) zeigen, dass der Effekt familiärer Armut auf Jugenddelinquenz mit der Konzentration sozialer Benachteiligungen im Wohngebiet anwächst. Schonberg/Shaw (2007) kommen in einer systematischen Forschungsübersicht zu dem Ergebnis, dass in einschlägigen Studien überwiegend eine puffernde Wirkung des elterlichen Verhaltens auf die Gefährdungen des sozialräumlichen Kontextes festgestellt wurde, die allerdings in Stadtvierteln mit extremen Benachteiligungen versagt. Wenn Eltern über die Freizeitaktivitäten und -orte ihrer Kinder unterrichtet sind, hat dies besonders in benachteiligten Wohngebieten einen abschwächenden Effekt auf deren Delinquenz (Lahey et al. 2008; vgl. auch Beyers et al. 2003; Brody et al. 2001; Browning/Leventhal/ Brooks-Gunn 2005; Rankin/Quane 2002; siehe Tab. 1, vgl. bereits Reckless/Dinitz/Murray et al. 1957). Qualitative Studien haben gezeigt, dass viele Eltern große Anstrengungen unternehmen, ihre Kinder von den Gefahren der von Gewalt geprägten Armenghettos in den amerikanischen Großstädten fernzuhalten (Furstenberg et al. 1999; Molnar et al. 2005; Pettit 2004). Einen zentralen Punkt der bekannten ethnographischen Studie Elijah Andersons (1999) in den Armenghettos in Philadelphia bildet die Unterscheidung zwischen den „,decent families “ und den „,street families “: Während die ersteren sehr viel Wert auf familiären Zusammenhalt und die Einhaltung konventioneller Werte und Normen auch unter widri- 
gen Umständen legen, stellen die letzteren der devianten Subkultur von Drogen und Gewalt nichts entgegen.

\section{Geschlecht}

$\mathrm{Zu}$ den überraschenden, aber inzwischen recht konsistenten Forschungsergebnissen gehört, das Mädchen tendenziell stärker vom Stadtviertelkontext geprägt werden als Jungen. Auswertungen der Chicagoer PHDCN-Daten zeigen, dass insbesondere die Gewaltdelinquenz von Mädchen stärker von räumlich konzentrierten Benachteiligungen beeinflusst wird als die der Jungen (Fagan/Wright 2012, siehe Tab. 1; Zimmerman/Messner 2010). In den am stärksten benachteiligten Wohnquartieren schließt sich die ansonsten recht große Lücke in der Gewaltneigung zwischen Mädchen und Jungen tendenziell. Ähnliche Ergebnisse werden auch aus anderen US-amerikanischen Studien berichtet (Elliott et al. 2006; Karriker-

Jaffe et al. 2009; Kling/Ludwig/Katz 2005; Kling/Liebman/Katz 2007; Vazsonyi/Cleveland/Wiebe 2006; Zahn/Browne 2009; siehe Tab. 1, vgl. Jones 2010; Ness 2004 für qualitative Studien). Einen gegenteiligen Befund in Bezug auf den Schulabbruch berichten jedoch Crowder/South (2003). Auch die Köln/Freiburger Studie hat starke sozialräumliche Kontexteffekte auf die Gewaltneigung von einheimischen Mädchen, nicht aber von Jungen gefunden - insbesondere in Hinblick auf die Zugehörigkeit zu gewaltorientierten Cliquen -, während bei Mädchen mit Migrationshintergrund ein entgegen gerichteter Effekt zu beobachten ist (Oberwittler 2003a; 2007b, siehe Tabelle 1). Während Mädchen aus Migrantenfamilien in den benachteiligten Stadtvierteln offenbar stärkeren familiären Kontrollen ausgesetzt sind, die ihre Gelegenheiten zu unbeaufsichtigten und riskanten Freizeitbeschäftigen begrenzen, gilt dies für einheimische Mädchen nicht. Bei ihnen scheint sich auszuwirken, dass der normative Kontext der Gleichaltrigen in den besonders benachteiligten Wohnquartieren Gewalthandlungen von Mädchen nicht stigmatisiert, wie es in den meisten anderen sozialräumlichen Kontexten der Fall ist. Damit übereinstimmend zeigte Kreager (2007, siehe Tab. 1) in einer Auswertung der schulischen Freundschaftsnetzwerke aus der „Add Health“-Studie, dass Gewalt von Mädchen in Schulen mit hohem generellen Gewaltniveau deren Populärität bei den Gleichaltrigen fördert, während gewalttätige Mädchen in allen übrigen Schulen weniger beliebt sind; bei Jungen fand sich dieser Interaktionseffekt zwischen Popularität und Gewaltniveau des Schulkontexts nicht. Die deutlich stärkeren Effekte auf Mädchen im „Moving to Opportunity“-Experiment haben 
weitere, detaillierte Auswertungen der Forscher provoziert (vgl. auch Burdick-Will/Ludwig in diesem Band). Im MTO-Experiment reagierten Mädchen deutlicher als Jungen mit einer Verringerung ihrer Gewaltdelinquenz auf den Umzug von einem extrem benachteiligten Stadtviertel in ein „besseres“ Wohnumfeld. Darüber hinaus nahm die Eigentumsdelinquenz der Jungen in der Experimentalgruppe sogar noch zu (Kling/Ludwig/Katz 2005, siehe Tab. 1).

Um diese erwartungswidrigen Folgen besser verstehen zu können, führten die Forscher qualitative Tiefeninterviews mit 86 der beteiligten Jugendlichen in Baltimore und Chicago durch (Clampet-Sundquist et al. 2011, siehe Tab. 1; vgl. Small/Feldman 2012). Es zeigte sich unter anderem, dass Jungen ihren Lebensstil weniger gut als Mädchen an die veränderten Erwartungen der Nachbarschaft anpassen konnten und ihre alten Freizeitgewohnheiten - vor allem das „Herumhängen“ mit Freunden auf der Straße - tendenziell beibehielten, was zu einer erhöhten Sichtbarkeit für die lokale Polizei und zu einer Stigmatisierung durch die Bewohner der „,besseren“ Wohnquartiere führte. Außerdem argumentierten die Forscher, dass den Jungen, die mit ihren Familien im Laufe der Zeit häufig in andere, wiederum benachteiligte Wohnquartiere umgezogen sind, die zur erfolgreichen „Navigation“ durch die Gefährdungen im urbanen Lebensumfeld notwendigen lokalen Kenntnisse und „Überlebensstrategien“ fehlten (ClampetSundquist et al. 2011: 1182, siehe Tab. 1).

\section{Gleichaltrige}

In den vorangehenden Abschnitten wurde bereits deutlich, dass die Beziehungen zu Gleichaltrigen offenbar eine wichtige Rolle als sozialer Mechanismus der Beeinflussung Jugendlicher durch sozialräumliche Kontexte spielen. Dies war zwar ein zentraler Bestandteil der ursprünglichen Desorganisationstheorie (Shaw/McKay 1969 [1942]), wurde aber unter anderem aufgrund Ruth Kornhausers (1978) einflussreicher Kritik der Subkultur-

theorien bis in die 1990er Jahre hinein unterbewertet (siehe oben). Seither spielen die Gleichaltrigenbeziehungen wieder eine wichtigere Rolle in der Forschung über Jugendkriminalität (Weerman 2011). Dazu hat unter anderem die „Add Health“-Studie beigetragen, die auch eine umfangreiche Netzwerkanalyse umfasst und damit vielfältige Analysemöglichkeiten bietet. Haynie/Silver/Teasdale (2006, siehe Tab. 1, vgl. Dupéré etal. 2008) zeigen mit diesen Daten, dass der Effekt sozialräumlicher Benachteiligungen auf 
Delinquenz weitgehend durch delinquente Gleichaltrigenkontakte vermittelt wird. Mit zunehmender sozialräumlicher Benachteiligung wächst die

Wahrscheinlichkeit des Kontaktes zu deviant orientierten Gleichaltrigen und sinkt die Wahrscheinlichkeit des Kontaktes zu konventionell orientierten Gleichaltrigen. Gemeinsame unbeaufsichtigte Freizeit lässt die Wahrscheinlichkeit delinquenter Handlungen in benachteiligten Wohngebieten eher ansteigen als in anderen Wohngebieten (Bernburg/Thorlindson 2007, siehe Tab. 1).

Entscheidend ist jedoch die Erkenntnis, dass nicht alle Jugendlichen in benachteiligten Wohngebieten selbst delinquent sind oder delinquente Freunde haben. Vielmehr haben Jugendliche trotz struktureller Beschränkungen auch in benachteiligten Stadtvierteln stets die Möglichkeit und auch die Notwendigkeit, zwischen verschiedenen „Angeboten“ auszuwählen, was Gleichaltrige, Freizeitbeschäftigungen, Freizeitorte und kulturelle Handlungsmuster betrifft. Als „kulturelle Heterogenität“ bezeichnet David Harding (2010) diese differenzierte Sichtweise, die die Vorstellung einer überwiegend oder gar einheitlich devianten Subkultur in benachteiligten Stadtvierteln zunehmend ablöst: "Adolescent boys in poor neighborhoods do not just follow the normative prescriptions and proscriptions of their immediate peer group but rather actively navigate and manipulate multiple sources of cultural messages “ (Harding 2010: 140). Diese Überlegungen (die natürlich nicht auf Jungen beschränkt sein können) decken sich mit den empirischen Befunden der Kölner/Freiburger Studie, wonach die räumliche Orientierung der Freundeskreise der Jugendlichen in allen untersuchten Stadtvierteln sehr heterogen ausfiel und klar damit im Zusammenhang stand, ob sie sich in ihrem Stadtviertel wohl fühlten oder nicht (Oberwittler 2004b). Nur ungefähr die Hälfte der befragten Jugendlichen hatte Freundeskreise, die überwiegend innerhalb des eigenen Wohnquartiers lokalisiert waren, die andere Hälfte war eher nach außen orientiert. Dies hing auch, aber bei weitem nicht nur mit der räumlichen Lage der Schule und der Verteilung der Mitschüler im Stadtgebiet zusammen. Die räumliche Auswahl der Freunde reflektiert vielmehr auch individuelle Präferenzen der Jugendlichen gegenüber den sozialräumlichen Milieus, in denen sie leben (vgl. Arum 2000). In der Auseinandersetzung der Jugendlichen mit ihrem Wohnquartier ist demnach ein Element der Wahl und der Selbstselektion enthalten, das in den sozialen Brennpunkten tendenziell zu einer Spaltung in „Anhänger“ und „Gegner“ des Wohnquartiers zu führen scheint. Es sind vor allem Hauptschüler, die sich eher lokal orientieren und in den sozialen Brennpunkten an der delinquenten Subkultur teilhaben.

Diese Erkenntnisse sind für die Frage der Stadtvierteleffekte auf das Verhalten von Jugendlichen deswegen höchst relevant, weil sich in der Kölner/Freiburger Studie zeigte, dass der sozialräumliche Kontext des Wohnquartiers nur für Jugendliche mit lokalem Freundeskreis 
messbare Auswirkungen hatte, während für Jugendliche mit nicht-lokalen Freunden keiner-

lei Zusammenhang zwischen sozialräumlicher Benachteiligung des eigenen Wohnquartiers und delinquentem Verhalten bestand (vgl. Friedichs 1998). Dies ist ein sehr starker Hinweis auf die Rolle der Gleichaltrigenbeziehungen als ein zentraler vermittelnder Prozess des sozialräumlichen Kontextes auf individuelles Verhalten von Jugendlichen. Hieraus ergeben sich auch methodische Probleme, denn offenbar reicht es für eine realistische Modellierung von sozialräumlichen Einflüssen nicht aus, die Wohnadresse zu kennen und die Bedingungen des Wohnquartiers zu berücksichtigen (siehe dazu unten).

\section{Methodische Aspekte der Erforschung sozialräumlicher Kontexteffekte}

Auf den restlichen Seiten möchte ich knapp einige zentrale methodische Probleme der Erforschung sozialräumlicher Einflüsse auf individuelles soziales Verhalten ansprechen, die jedoch so vielfältig und komplex sind, dass ihre angemessene Behandlung in einen einführenden Beitrag unmöglich ist. Die Spezialliteratur zu den methodischen Aspekten sozialräumlicher Kontexteffekte ist inzwischen recht vielfältig (Booth/Crouter 2001; Diez Roux 2004; Diez Roux/Mair 2010; Duncan/Raudenbush 1999; Duncan/Murnane 2011; Friedrichs 1998; Friedrichs/Galster/Musterd 2003; Leventhal/Brooks-Gunn 2000; Sampson/Morenoff/Gannon-Rowley 2002; van Ham et al. 2012; vgl. auch die Beiträge von Simonson und Nonnenmacher in diesem Band).

Kontext- oder Individualeffekte?

Die grundlegende Fragestellung der Studien zu sozialräumlichen Kontexteffekten lautet: Haben Kontexte eine eigenständige kausale Bedeutung für das soziale Verhalten von Menschen, unabhängig von allen relevanten individuellen Einflussfaktoren? Ein Kontexteffekt durch die sozialräumliche Konzentration von sozialen Benachteiligungen (oder anderen ungünstigen kollektiven Eigenschaften des Sozialraumes) liegt nur dann vor, wenn diese Wirkungen die 
Summe aller individuellen Wirkungen sozialer Benachteiligungen übersteigen. Anderenfalls wäre es lediglich ein Kompositionseffekt, d.h. die soziale Zusammensetzung der Individuen in einem Kontext alleine würde bereits ausreichen, um das Ausmaß des untersuchten Problemverhaltens in diesem Kontext zu erklären (die Begriffe Kontext- und Kompositionseffekt werden jedoch teilweise synonym verwendet).

Eine solche Fragestellung lässt sich nur auf der Basis quantitativer, systematisch erhobener Daten beantworten und setzt ein geeignetes statistisches Verfahren der simultanen Schätzung individueller und kollektiver Einflüsse auf eine abhängige Variable voraus - die Mehrebenenanalyse (s.u.). Das bedeutet nicht, dass qualitative und ethnographische Verfahren keine Bedeutung für die Forschung zu Nachbarschaftseffekten hätten. Insbesondere wenn es um soziale Mechanismen der Vermittlung von Kontexteffekten auf individuelles Verhalten und die Frage geht, wie Menschen ihre Lebensumwelt wahrnehmen und in ihr „navigieren“, leisten qualitative Studien einen sehr wichtigen Beitrag zur Forschung, der auch von der

quantitativ ausgerichteten Forschung anerkannt und aufgegriffen wird. Mittlerweile „klassische“ Studien aus der jüngeren Zeit sind etwa Elijah Andersons (1999) Studie zum „Street Code“ in den Ar- menghettos von Philadelphia und Mary Pattillos (1999) Ethnographie der Sozialkontrolle in einem schwarzen Mittelschichtsviertel in Chicago (vgl. auch Carr 2005; Harding 2010; St. Jean 2007).

Um den eigenständigen Effekt sozialräumlicher Kontexte zu isolieren, müssen die relevanten individuellen, mit den Eigenschaften der Makro-Ebene möglicherweise konfundierten Einflussfaktoren kontrolliert werden. Dabei tritt häufig das aus der multivariaten Datenanalyse allgemein bekannte Problem der unzureichenden Kontrolle von Drittvariablen auf (, unobserved heterogeneity“ oder ,omitted variable bias“), welches zu einem Überschätzen des Kontexteffekts führen kann (Wolff/Best 2010). Besonders heikel ist dies deswegen, weil wenig über den Prozess der (Selbst-)Selektion von Individuen in soziale Kontexte bekannt ist (Elwert/Winship, im Druck). Im Falle von Kindern und Jugendlichen betrifft dies zunächst die Selektion der Eltern in Wohnquartiere (Duncan/Raudenbush 1999: 36; Leventhal/BrooksGunn 2000: 314). Falls bestimmte Eigenschaften der Familien, die kausal auch auf das Verhalten der Jugendlichen wirken, für die Auswahl der Wohnquartiere durch die Eltern mitverantwortlich sind, sollten diese auch im Modell berücksichtigt werden, um Scheinkorrelationen zwischen den Stadtvierteleigenschaften und dem jugendlichen Verhalten zu vermeiden. Duncan/Raudenbush (1999: 36) nennen als Beispiel die Sorge der Eltern um die Entwicklung ih- 
rer Kinder. Wenn besorgte Eltern, die einen kontrollierenden und autoritativen Erziehungsstil haben, sozial benachteiligte Stadtviertel wegen möglicher Gefahren für ihre Kinder meiden, während andere Eltern einen eher vernachlässigenden Erziehungsstil haben und zugleich auch in benachteiligten Stadtvierteln wohnen, dann könnte der vermeintliche Stadtvierteleffekt ein Effekt des individuellen elterlichen Erziehungsstils sein, und es würde sich um einen Fall von Endogenität handeln (Duncan/Magnuson/Ludwig 2004). Noch deutlicher tritt dieses Problem bei Studien zum Einfluss des Schulkontextes auf Jugendliche hervor: Insbesondere das deutsche Schulsystem ist mit dem Übergang von der Primar- zur Sekundarschule sehr selektiv, und problematische Verhaltensformen von Schülern beispielsweise in der 8. Jahrgangsstufe, die Forscher als potenzielles Ergebnis der Kontextbedingungen einer Hauptschule interpretieren wollen, könnten bereits in der 4. Jahrgangsstufe dazu geführt haben, dass diese Schüler auf eine Hauptschule und nicht auf ein Gymnasium gekommen sind. Persönlichkeitsmerkmale, die dem Problemverhalten zugrunde liegen, wären in diesem Fall dem Kontext kausal vorgelagert und sollten dann im Modell berücksichtigt werden. Harding (2003) hat jedoch gezeigt, dass die Gefahr der massiven Überschätzung von Stadtvierteleffekten auf Schulabbruch und Teenage-Schwangerschaften durch unbeobachtete Eltern-Eigenschaften zumindest unwahrscheinlich ist. Ein fortgeschrittenes technisches Verfahren der Drittvariablenkontrolle, das in den letzten Jahren dabei häufiger zum Einsatz kommt, jedoch von der Verfügbarkeit entsprechend reicher Datenbestände abhängig ist, ist das „propensity score matching “ (Dehija/Wahba 2002; Sampson/Sharkey/Raudenbush 2008, siehe Tab. 1).

Es besteht umgekehrt jedoch auch die Gefahr des „Über-Kontrollierens“ (over controlling) individueller Merkmale, die dazu führen kann, dass tatsächlich existente sozialräumliche Kontexteffekte nicht mehr erkannt werden (Sampson/Morenoff/Gannon-Rowley 2002: 467; Wodtke/Harding/Elwert 2011: 714). Dies trifft dann zu, wenn individuelle Merkmale als Kontrollvariablen verwendet werden, die selbst das Ergebnis der Beeinflussung durch den Kontext sind. Zum Beispiel ist die Existenz delinquenter Freunde, ein häufig verwendeter und robuster Prädiktor von Jugenddelinquenz, möglicherweise stark beeinflusst durch das „Angebot" an Gleichaltrigen im Wohnquartier und daher eine Mediatorvariable des Einflusses des sozialen Kontextes auf das delinquente Verhalten. Diese Variable würde bereits einen Teil der Varianz erklären, die letztlich dem Kontext zuzurechnen ist, auch wenn sie als Mediatorvariable auf der individuellen und der Stadtviertelkontext auf der kollektiven Ebene des Mehrebenenmodells zu verorten ist (s.u.). Ähnliches gilt für Einstellungsskalen wie deviante Normori- 
entierungen, die sich im Laufe der Sozialisation von Kindern und Jugendlichen auch unter dem Einfluss sozialräumlicher Kontexte herausbilden. Einige Studien untersuchen sogar, inwieweit eine niedrige Selbstkontrolle, also ein individualpsychologisches Merkmal, von dem Gottfredson/Hirschi (1990) annahmen, dass es über die gesamte Lebensspanne hinweg stabil sei, als Ergebnis sozialräumlicher Kontexteinflüsse während des Kindes- und Jugendalters zu betrachten ist (Beaver/Wright/Maume 2008; Teasdale/Silver 2009).

Letztlich kann es keine vollständige Trennung von Individual- und Kontexteffekten geben, denn zu einem gegebenen Zeitpunkt sind viele relevante individuelle Merkmale potenziell mit beeinflusst von vorhergegangenen sozialen Erfahrungen, die Kinder und Jugendliche in sozialräumlichen Kontexten gesammelt haben. Angesichts dieser Problematik erscheint es als sehr ratsam, die Ergebnisse von Querschnittsstudien vorsichtig zu interpretieren und eher von ,potenziellen“ denn von ,realen“ Kontexteffekten auszugehen.

Quer- oder Längschnittstudien?

In der Konsequenz ergibt sich aus dem zuvor Gesagten, dass längsschnittliche Studiendesigns für die Identifizierung von Kontexteffekten (wie für die Identifizierung von Kausalbeziehungen ganz generell) überlegen sind, insbesondere wenn es um Wohnquartiere als Entwicklungskontexte von Kindern und Jugendlichen geht. Die in der aktuellen Forschung am häufigsten verwendeten Datensätze (,Add Health“ und PHDCN) haben ein Längsschnittdesign, und Analyseergebnisse können dementsprechend in Hinblick auf den Einfluss sozialräumlicher Faktoren auf Veränderungen im Verhalten interpretiert werden - unter Kontrolle des jeweiligen Ausgangsniveaus. Dieser Analyseansatz kann zwar das grundlegende Problem der Abgrenzung von individuellen und kontextuellen Einflüssen nicht vollständig lösen, jedoch sind Aussagen über kausale Effekte insbesondere über kürzere Zeiträume doch stärker belastbar, als es in reinen Querschnittsstudien der Fall ist.

Darüber hinaus kann nur in längsschnittlichen Studien untersucht werden, ob sozialräumliche Kontexte eher kurzfristige oder langfristige Wirkungen auf Kinder und Jugendliche entfalten, und welche Bedeutung wechselnde räumliche Kontexte und die Kumulation

verschiedener so- zialräumlicher Erfahrungen haben. Einige amerikanische Studien zeigen, 
dass sich ungünstige sozialräumliche Bedingungen in der Kindheit vor allem langfristig negativ auf verschiedene

psycho-soziale Dimensionen auswirken. Wheaton/Clarke (2003) berechneten, dass der zeitverzögerte und kumulative Effekt sozialräumlicher Benachteiligungen während der Kindheit auf psychische Belastungen im frühen Erwachsenenalter stärker ist als der gegenwärtige Effekt des Wohnquartiers (und dass dieser Kontexteffekt in Familien mit niedriger elterlicher Bildung wesentlich stärker ausgeprägt ist als in anderen Familien). Mit den Daten der „Panel Study of Income Dynamics “ zeigen Wodtke/Harding/Elwert (2011, vgl. Jackson/Mare 2007; Timberlake 2007) ebenfalls unter Berücksichtigung von wechselnden Wohnumgebungen, dass die Wahrscheinlichkeit eines College-Abschlusses bei schwarzen Jugendlichen mit zunehmender sozialräumlicher Benachteiligung um 20 Prozentpunkte fällt (bei anderen Jugendlichen um acht Prozentpunkte). Schließlich finden Sharkey/Elwert (2011) sogar empirische Hinweise auf eine intergenerationelle Übertragung sozialräumlicher Benachteiligungen auf Schulleistungen: Wenn die Eltern vor der Geburt ihrer Kinder in benachteiligten Wohnquartieren gelebt haben, hat dies einen eigenständigen Effekt unter Kontrolle der Kontextbedingungen, in denen die Kinder leben, sowie individueller Merkmale. Insgesamt schätzen Sharkey/Elwert (2011: 1964) den Kontexteffekt der früheren und gegenwärtigen Sozialräume auf mehr als eine halbe Standardabweichung der Sprachkompetenz. Dieser sehr substantielle Effekt steht im Gegensatz zu einem nur minimalen Kontexteffekt, den Brännström/Rojas (2012) in einer der ganz wenigen vergleichbaren europäischen Studien in Schweden gefunden haben. Es ist angesichts der unterschiedlichen Gesellschaftssysteme plausibel, dass sozialräumliche Benachteiligungen in den USA wesentlich stärkere Auswirkungen haben als in Schweden (Brännström 2006).

Wohnquartiere und/oder Schulen?

In den theoretischen Überlegungen und empirischen Befunden wurde bereits deutlich, dass Schulen (oder auch Schulklassen) neben Wohnquartieren einen zweiten, sehr relevanten sozialräumlichen Kontext für die soziale Entwicklung von Kindern und Jugendlichen darstellen. Besonders bei Studien zum Bildungserfolg und zum Sozialverhalten in der Schule liegt es nahe, anstelle von Wohnquartieren den Einfluss des schulischen Kontextes zu untersuchen, wie dies Michael Rutter (1979) in seiner bahnbrechenden Studie „Fifteen thousand hours“ getan hat, und wie dies auch neuere Studien zum Bildungserfolg (Ditton/Krüsken 2006) und zur schulischen Delinquenz (Fuchs/Schmalz 2010) tun. Trotzdem verwenden viele Studien 
zum Bildungserfolg, insbesondere in den USA, Wohnquartiere und nicht Schulen als Kontexteinheiten (Harding et al. 2011; Sampson/Sharkey/Raudenbush 2008; Sharkey/Elwert 2011; Wodtke/Harding/Elwert 2011). Dem Wohnquartier wird damit eine Art sozialräumlicher, dem Schulkontext übergeordneter „Master-Status“ zugeschrieben (auch wenn die Ausblendung des Schulkontextes lediglich einem Mangel an verfügbaren Daten geschuldet sein sollte). Dies erscheint jedoch nur insofern sinnvoll, als die kommunale Organisation der Schulen eine

weitgehende räumliche Überlappung von Wohngebieten und Schulbezirken herstellt, was offenbar auch auf Island zutrifft (Bernburg/Thorlindsson 2005, 2007, siehe Tab. 1), jedoch bestimmt nicht auf Deutschland. Bereits in den deutschen Grundschulen übersteigt aufgrund des Schul- wahlverhaltens der Eltern das Maß der ethnischen und sozialen Segregation in den Schulen das in den Wohnquartieren (Fincke/Lange 2012; Kristen 2008), und durch das in den meisten Bundesländern fortbestehende dreigliedrige Sekundarschulsystem ist die schulische Segregation im Jugendalter nochmals deutlich stärker ausgeprägt (Oberwittler 2007a), mit vieldiskutierten negativen Folgen insbesondere für die Hauptschüler (Solga/Wagner 2008). Die theoretisch naheliegende Annahme (s.o.), dass sowohl der Wohnquartiers- als auch der Schulkontext gleichzeitig Bedeutung für Kinder und Jugendliche haben könnte, führt zu zusätzlichen methodischen Problemen, da nicht nur geeignete Daten für beide Kontexte vorliegen müssen, sondern auch die statistische Modellierung in Form von so genannten kreuzklassifizierten Mehrebenenanalysen noch komplexer wird. Die bislang noch wenigen Studien, die mithilfe dieser Methode gleichzeitige Einflüsse von Wohnquartieren und Schulen untersuchen, deuten mehrheitlich auf stärkere oder sogar ausschließliche Schulkontexteffekte hin. Dieses Ergebnis überrascht nicht, wenn es um Schulleistungen oder schulisches Verhalten geht (Brännström 2008; Goldsmith 2009; Kauppinen 2008, Leckie 2009; Rasbash et al. 2010; Sykes/Musterd 2011), zeigte sich ähnlich aber auch in Analysen außerschulischer Delinquenz. In der Kölner/Freiburger Studie war der Effekt der Konzentration sozialer Benachteiligungen auf schwere Delinquenz auf der Schulebene ca. doppelt so stark wie der simultan berücksichtigte entsprechende Effekt auf der Wohnquartiersebene (Oberwittler 2007b, 799; vgl. Pauwels in diesem Band). Jedoch stehen diese Ergebnisse insbesondere auf der Basis von Querschnittsdaten unter dem methodischen Vorbehalt der Verzerrungen durch Selektionseffekte, die den schulischen Kontext stärker betreffen als den Wohnquartierskontext (s.o.). Weiterhin ungeklärt bleiben auch Fragen nach möglichen Wechselwirkungen zwischen beiden Kontex- 
ten, beispielsweise ob positive Einflüsse des Schulkontextes Gefährdungen durch den Wohnquartierskontext neutralisieren können.

\section{Aktionsräume und Agency von Kindern und Jugendlichen}

Eine besondere methodische Herausforderung, die von den wenigsten empirischen Studien adäquat gelöst oder überhaupt aufgegriffen wird, liegt in der Umsetzung der theoretisch leicht $\mathrm{zu}$ formulierenden Erkenntnisse, dass Wohnquartiere keine Inseln und Kinder und Jugendliche keine passiven Opfer ihrer Lebensumwelt sind. Nicht nur wenn es um die Schule geht, sondern auch in der Freizeit sind Kinder und vor allem Jugendliche in ihren Aktionsräumen nicht auf ihr eigenes Wohnquartier beschränkt. Eine der wichtigsten Ergebnisse der Kölner/Freiburger Studie war, dass viele Jugendliche einen wichtigen Teil ihrer Freizeit außerhalb ihres eigenen Wohnquartiers verbringen, verbunden mit der Auswahl ihrer Freundeskreise und ihren persönlichen Raumpräferenzen, und dass dieses Raumverhalten über die Wirkung der Sozialräume auf die Jugendlichen mitentscheidet (Oberwittler 2004b, vgl. Rabold/Baier in diesem Band).

Kinder und Jugendliche sind den sozialräumlichen Bedingungen ihres Wohnumfeldes also nicht hilflos ausgeliefert, sondern verfügen über ein bestimmtes Maß an Agency, also individueller Entscheidungs- und Handlungsmacht (Lange 2008). Dies gilt wahrscheinlich nicht im gleichen Maße für US- oder lateinamerikanische Großstädte wie für europäische Großstädte mit ihren erheblich geringeren sozialen Ungleichheiten und besse- ren urbanen Infrastrukturen. Und auch wenn man die Vorstellung der jugendlichen Agency akzeptiert, stellt sich dennoch die Frage nach den individuellen und familiären Faktoren, die die Jugendlichen in unterschiedlichem Maße dazu befähigen, selbständig über ihre Aktionsräume zu bestimmen.

Die meisten Studien, insbesondere solche mit großen und national-repräsentativen Stichproben, beschränken die Messung der sozialräumlichen Bedingungen jedoch auf die Zuordnung der Wohnadressen der Befragten zu kleinräumlichen Verwaltungsbezirken und das „Zuspielen" der jeweiligen soziodemographischen Kontextdaten. Damit wird die falsche Annahme einer ausschließlichen Beeinflussung durch die eigene Wohnumgebung in unzureichende em- 
pirische Modelle überführt, die individuelle Unterschiede im Raumverhalten nicht abbilden können. Eine innovative Ausnahme im Feld der Jugenddelinquenzforschung stellt die englische „Peterborough Adolescent and Young Adult Development Study“ (PADS+) dar, die für jeweils einige Tage auch die räumliche Dimension der Tagesabläufe der Befragten in detaillierten Protokollen erfasst und so ein wesentlich genaueres Bild der zeitlichen und räumlichen „Aktivititätsfelder“ von Jugendlichen gewinnen kann (Wikström et al. 2010, 2012). Inzwischen werden auch GPS-gestützte Aufzeichnungen der räumlichen Mobilität von Jugendlichen für die Forschung genutzt (z.B. Wiehe et al. 2008). Dadurch ergeben sich neue Möglichkeiten zur detallierten Analyse von räumlichem Verhalten, allerdings stellt diese Datenfülle auch neue Herausforderungen an geeignete statistische Modellierungen.

\section{Die Messung von Kontexteigenschaften}

Seit der Phase der klassischen Desorganisationstheorie ab den 1930er Jahren bis heute dienen einige soziodemographische Merkmale als zentrale Indikatoren sozialräumlicher Problemlagen. Die räumliche Konzentration sozialer Benachteiligungen wird üblicherweise gemessen, indem die Anteile von Bewohnern mit niedrigem Einkommen, von Arbeitslosen oder Empfängern von Sozialleistungen, von Bewohnern mit Migrationshintergrund oder ausländischer Staatsangehörigkeit, den Anteil von Alleinerziehenden etc. berechnet werden. Die durchschnittliche Wohndauer der Bewohner, die Bevölkerungsdichte, die Gebäudestruktur und Flächennutzungsdaten -werden häufig als Indikatoren der Urbanität oder Zentralität von Wohnquartieren verwendet. In Deutschland basieren diese Daten auf den Einwohnermelde- und weiteren Registern der Kommunalverwaltungen, in anderen Ländern wie Großbritannien und den USA eher auf den Volkszählungen. In beiden Fällen handelt es sich um Vollerhebungen, also um reliable Datenquellen mit sehr geringen Messfehlern, die

in vielen Großstädten klein- räumlich verfügbar sind. Sie sind daher wesentlich besser zur Messung der sozialräumlichen Kontextbedingungen geeignet als Befragungsdaten. Geht es jedoch nicht nur um die soziodemographische Struktur, sondern auch um kollektive soziale Eigenschaften und Prozesse von Wohnquartieren, so gibt es zu Befragungsdaten kaum eine Alternative. Dass diese theoretisch schon lange interessierenden kollektiven Eigenschaf- 
ten durch Befragungsdaten und innovative statistische Methoden der empirischen Analyse zugänglich gemacht wurden, stellt einen ganz wesentlichen Fortschritt in der Forschung in den letzten ca.15 Jahren dar. Raudenbush/Sampson (1999, vgl. Oberwittler 2003b) haben auf der Basis der Varianzzerlegung in der Mehrebenenanalyse ein statistisches Verfahren zur Reliabilitätsprüfung solcher Befragungsdaten auf der kollektiven Ebene entwickelt, das sie in Anlehnung an psychometrische Methoden ,ecometrics“ nennen. Die Intraklassenkorrelation (ICC) und der Koeffizient Lambda geben darüber Auskunft, wie übereinstimmend Befragte innerhalb eines Kontexts kollektive Merkmale dieses Kontexts bewerten. Je ähnlicher deren Einschätzungen beispielsweise zur sozialen Kohäsion im Wohnquartier oder zum Lehrer-Schüler-Verhältnis in einer Schule sind (d.h. je kleiner die Binnengruppenvarianz ist), und je mehr sich auch Wohnquartiere oder Schulen in diesen Bewertungen unterscheiden (d.h. je größer die Zwischengruppenvarianz ist), desto wahrscheinlicher ist es, dass mit den Befragungsdaten kollektive Kontextmerkmale reliabel gemessen werden. Die ICCs der Skalen zur kollektiven Wirksamkeit, zur Kriminalitätsfurcht oder zur Wahrnehmung von Unordnung in Wohnquartieren erreichen üblicherweise Werte von ca. 15 bis 25\% (Oberwittler 2003b; Pauwels/Hardyns/Van der Velde 2010; vgl. Häfele, Peter/Schlepper/Lüdemann in diesem Band). Mithilfe von Mehrebenenmodellen können dabei auch kontextbezogene Schätzwerte gebildet werden, die um Kompositionseffekte (z.B. aufgrund unterschiedlicher Alters- oder Bildungsstrukturen in Wohnquartieren) bereinigt sind, oder die gemäß den Prinzipien der Bayes'schen Statistik für geringe Reliabilität korrigiert werden, indem sie an den Gesamtmittelwert der Stichprobe angenähert werden (z.B. Sampson/Raudenbush/Earls 1997: vgl. Oberwittler/Wikström 2009).

Dennoch ist die Messung von Kontexteigenschaften durch Befragungsdaten nicht unproblematisch, wenn sowohl diese Kontexteigenschaften als unabhängige Variable als auch das zu erklärende Phänomen als abhängige Variable durch die Befragung derselben Personen gemessen werden. Ein Beispiel dafür ist der Einfluss der wahrgenommenen Unordnung im Wohnquartier auf die Kriminalitätsfurcht der befragten Bewohner, oder der Einfluss des wahrgenommenen Schulklimas auf das schulische Verhalten der befragten Schüler. Weniger problematisch ist die Aggregierung objektiver individueller Merkmale wie Geschlecht oder Migrationsstatus (Lüdtke et al. 2008). Zwar sind die aggregierten Befragungsdaten über-individuell, dennoch ist mit Messfehlerkorrelationen zwischen den unabhängigen und der abhängigen Variablen zu rechnen, die zu einer Überschätzung der Zusammenhänge führen können (Duncan/Raudenbush 1999, 2001). So fanden Taylor und seine Mitautoren, dass Befragte, die furchtsamer sind, auch mehr Unordnung im Wohnquartier berichten, so dass der Zusammenhang zwischen Incivilities und Kriminalitätsfurcht oft überschätzt wird (Covington and Taylor 1991; Perkins/Taylor 1996; Taylor 2001: 228, s.o.). Für die Schulforschung haben 
Lüdtke/Robitzsch/Köller (2002) in Simulationsrechnungen gezeigt, dass Kontexteffekte,

die durch Aggregieren der individuellen Antworten von Schülern gemessen werden, je nach Reli- abilität dieser Messungen statistische Artefakte sind.

Eine Lösung dieses Problems liegt in unabhängigen Messungen der Kontexteigenschaften und der Zielvariablen (Duncan/Raudenbush 1999, 2001). Zum Beispiel wurden im Chicagoer Projekt PHDCN zur Messung des Verhaltens der Jugendlichen und der kollektiven Wirksamkeit der Wohnquartiere zwei getrennte Befragungen durchgeführt. Ebenso werden in der pädagogischen Forschung kollektive schulische Prozesse durch Lehrerbefragungen gemessen, die den Befragungs- oder Testdaten der Schüler zugespielt werden. Wenn es um die Messung von

Unordnung in Wohnquartieren geht, sind unabhängige systematische Beobachtungen durch geschulte Personen der Erhebung innerhalb der Bewohnerbefragung vorzuziehen (Raudenbush/Sampson 1999b, vgl. Häfele in diesem Band). Oder zusätzlich zur Wohnbevölkerung können ,lokale Experten“ wie z.B. Geschäftsleute, Sozialarbeiter oder Polizisten zu den kollektiven Eigenschaften der Wohnquartiere befragt werden. Pauwels/Hardyns (2009) haben gezeigt, dass so mit weniger als zehn Befragten pro Raumeinheit sehr reliable Daten zu erhalten sind. Auf der statistischen Ebene könnten neue Ansätze von Mehrebenenmodellen mit latenten Variablen, analog zu herkömmlichen Strukturgleichungsmodellen, zu einer Lösung der Messfehlerproblematik beitragen (Lüdtke et al. 2008, Marsh et al. 2009, Raudenbush/Johnson/Sampson 2003; Raudenbush/Sampson 1999a; siehe unten).

Statistische Modellierung von Kontexteffekten

Der Aufschwung des Forschungsfeldes sozialräumlicher Kontexteffekte auf Kinder und Jugendliche ist nicht nur mit der erneuten Popularität sozialstruktureller Fragestellungen zu erklären, sondern auch entscheidend mit der Entwicklung einer neuen statistischen Methode, der Mehrebenenanalyse, verbunden, welche die Probleme und Defizite früherer Analyseansätze erfolgreich überwinden konnte (Baumer/Arnio 2012; De Leeuw/Meijer 2008; DiPetre/Forristal 1994; Hox 2010; Hox/Roberts 2009;.Langer 2004; Snijders und/ Bosker 2012; Raudenbush und/ Bryk 2002; vgl. auch Simonson in diesem Band). Die Mehrebenenanalyse 
erweitert die klassische Regressionsanalyse durch die Berücksichtigung der gruppierten Ordnung von Stichproben. Dadurch können Effekte eines gemeinsamen Kontextes bei simultaner Berücksichtigung individueller Einflüsse der Befragten korrekt geschätzt werden. Zum Beispiel kann der soziale Status sowohl als Individualmerkmal und gleichzeitig in Form des durchschnittlichen Sozialstatus eines Wohnquartiers als Gruppenmerkmal in ein Mehrebenenmodell aufgenommen werden. Hat der durchschnittliche Sozialstatus des Wohnquartiers einen signifikanten Effekt z.B. auf die Delinquenz der Jugendlichen unter Kontrolle des individuellen Sozialstatus der Jugendlichen, so deutet dies auf einen Kontexteffekt hin. Beispiele aus der pädagogischen Forschung zeigen, dass dasselbe Phänomen auf individueller und kollektiver Ebene sogar entgegengesetzte Wirkungen entfalten kann. So wirkt sich die individuelle schulische Leistungsfähigkeit positiv und die durchschnittliche schulische Leistungsfähigkeit in der Klasse aufgrund von Vergleichsprozessen negativ auf das schulische Selbstkonzept aus - der so genannte ,big-fish-little-pond

effect" (Marsh et al.2007). Bernburg/Thorlindson/Sifusdottir (2009) errechneten in analoger Weise, dass individuelle ökonomische Deprivation bei Jugendlichen vor allem dann zu Frustrationen und delinquentem Verhalten führt, wenn die anderen Jugendlichen in ihrer Umgebung nicht depriviert sind.

Die Mehrebenenanalyse berücksichtigt die hierarchische Datenstruktur von Individuen in gemeinsamen Kontexten durch eine Zerlegung der Varianz in eine individuelle Komponente (Unterschiede zwischen Befragten innerhalb ihrer Gruppen) und eine kollektive Komponente (Unterschiede zwischen den Gruppen) (vgl. Simonson in diesem Band). Gleichzeitig stellt die Mehrebenenanalyse auch sicher, dass die unterschiedlichen Stichprobengrößen der Einheiten auf den verschiedenen Ebenen bei der Berechnung der Standardfehler berücksichtigt werden. Während die Standardfehler der Koeffizienten auf der höheren Ebene in konventionellen Regressionsmodellen unterschätzt würden, vermeidet die Mehrebenenanalyse diesen Fehler durch die simultane Schätzung mehrerer Gleichungen auf der Basis der jeweils gültigen Zahl der Einheiten (z.B. 2000 Befragte in 40 Stadtvierteln).

Auch Erweiterungen auf drei oder mehr hierarchische Ebenen (z.B. Jugendliche in Familien in Stadtvierteln) sowie kreuzklassifizierte Modelle (Jugendliche in Schulen und unabhängig davon in Stadtvierteln) und weitere komplexere Gruppierungen sind möglich. Längsschnittdaten werden häufig so modelliert, dass die Erhebungszeitpunkte als unterste Ebene, die zeitkonstanten Werte der Personen als zweite und räumliche Kontexte als dritte Ebene definiert 
werden. Studiendesigns, in denen die räumliche Struktur der Wohnquartiere der Befragten durch die Berücksichtigung individuell unterschiedlicher Aktionsräume oder von Umzügen über längere Zeitspannen hinweg aufgebrochen wird, können dagegen nur schwerlich in Mehrebenenmodellen abgebildet werden (vgl. Burdick-Will/Ludwig in diesem Band). Da die Mehrebenenanalyse noch recht neu ist und zumal in Deutschland noch nicht sehr häufig angewendet wird, bestehen in der Forschungspraxis vielfach Unsicherheiten hinsichtlich einer Reihe von Fragen. Dies beginnt mit dem Stichprobendesign, insbesondere mit der sinnvollen Anzahl der Gruppenkontexte. Viele traditionelle Studien zu sozialräumlichen Phänomenen sind so angelegt, dass jeweils eine relativ große Zahl von Personen in einer sehr geringen Anzahl von Wohnquartieren untersucht werden (z.B. Friedrichs/Blasius 2000; Heitmeyer et al. 2011). Unterschiede zwischen zwei oder drei Wohnquartieren können dann allerdings nur deskriptiv interpretiert werden, da diese Anzahl nicht für die Verwendung von Prädiktoren auf der Kontextebene ausreicht. Die Anforderungen der Mehrebenenanalyse an die Stichprobengröße auf der Kontextebene unterscheiden sich nämlich nicht grundsätzlich von den Anforderungen an Stichproben in der konventionellen Regressionsanalyse (Snijders/Bosker 2012: 177). Das bedeutet, dass auch 20 oder 30 Kontexte noch eine sehr geringe Anzahl darstellen, bei denen multivariate Modelle mit mehr als einem Prädiktor schnell an ihre Grenze kommen (vgl. jedoch für Länder als Kontexte Hox/ van de Schoot/Matthijsse. 2012). Wesentlich besser sind Stichprobendesigns mit sehr vielen Kontexten. Hat man die Wahl zwischen 20 Kontexten mit jeweils 100 Befragten und 100 Kontexten mit jeweils 20 Befragten, so ist die zweite Alternative statistisch deutlich überlegen, da der Zugewinn an statistischer Power bei einer Zunahme von Kontexten größer ist als bei einer Zunahme

von Befragten, die in der Mehrebenenanalyse über die Kontexten gepoolt werden (Murray/Varnell/Blitstein

2004: 424; Snijders/Bosker 2012: 184). Oberwittler/Wikström (2009) demonstrieren dies mit den Daten einer Bewohnerbefragung im Rahmen der PADS+-Studie, bei der die Stichprobe in 518 sehr kleinen Wohnquartieren mit durchschnittlich nur 13 Befragten organisiert ist. Das damit im Zusammenhang stehende Problem der Größe und des Zuschnitts von Gebietseinheiten, die als Wohnquartiere definiert werden, soll hier nur gestreift werden (siehe dazu Nonnenmacher in diesem Band; vgl. Oberwittler/Wikström 2009; Robitaille et al. 2011). Die grundsätzlich richtige Annahme, dass kleinere räumliche Einheiten aufgrund ihrer größeren 
internen Homogenität bessere Voraussetzungen für die Identifizierung von Kontexteffekten bieten; gilt offenbar nicht absolut und könnte auch davon abhängen, wie klein- oder großflächig städtische Siedlungsstrukturen beschaffen sind. und wie Bewohner diese wahrnehmen. Es kommt hinzu, dass benachbarte Wohnquartiere indirekte „Ausstrahlungs“-Effekte ausüben könnten, die als so genannte räumliche Autokorrelationen in statistische Modelle integrierbar sind (Anselin 2002; Bernasco/Elffers 2009; Ceccatto/Oberwittler 2008; O'Brien Caughy/Hayslett-McCall/O'Campo 2007; Verbitsky-Savitz/Raudenbush 2009). Eine weitere, in der Literatur zur Mehrebenenanalyse selten thematisierte Frage betrifft die Zentrierung der Variablen auf der untersten Ebene (Enders/Tofighi 2007; Paccagnella 2006; Raudenbush/Bryk 2002: 134-149). Eine Zentrierung der Prädiktoren auf der unteren Ebene um den jeweiligen Gruppenmittelwert führt zu einer grundlegend anderen Interpretation der Koeffizienten als eine Zentrierung um den Gesamt-Mittelwert aller Gruppen (grand mean). Während sich die erstere Variante zur stabileren Modellierung von Kontexteffekten auf die Varianz von individuellen Zusammenhängen (so genannte slope-as-outcome-Modelle) eignet, ist die zweite Variante angemessen, wenn es um die Effekte von Kontextbedingungen auf die

durchschnittliche Ausprägung der Zielvariablen geht (so genannte random intercept-Modelle). Daher sollte die Wahl des Zentrierungsverfahrens stets dokumentiert und begründet werden.

Häufig ist Unklarheit bei der Frage anzutreffen, wie bestimmte unabhängige, auf den Kontext bezogene Variablen (z.B. die wahrgenommene soziale Kohäsion im Wohnquartier) in ein Mehrebenmodell aufgenommen werden sollten: Entweder nur auf der individuellen Ebene (L1), oder nur auf der räumlichen Kontextebene (L2), oder aber auf beiden Ebenen gleichzeitig? Eine allgemeingültige Regel dafür gibt es zwar nicht, aber grundsätzlich kann man sich an der theoretischen Verortung der Variable orientieren: Eine primär individuell wirksame Variable (wie z.B. der Bildungsstatus) sollte in jedem Fall auf der individuellen Ebene ins Modell eingeführt werden. Sie kann zusätzlich aber auch auf der kollektiven Ebene als aggregiertes Kontextmerkmal des Wohnquartiers aufgenommen werden, wenn z.B. die Hypothese getestet werden soll, dass über den Kompositionseffekt hinausgehende Kontexteffekte des durchschnittlichen Bildungsstatus bestehen. Bei kollektiv verorteten Eigenschaften (wie z.B. der sozialen Köhasion im Wohnquartier) macht eine Aufnahme ins Mehrebenenmodell auf der individuelle Ebene (L1) weniger Sinn, da die Wirkung dieser kollektiven Eigenschaft theoretisch auf der kollektiven Ebene (L2) erwartet wird, und dieser 
Effekt entsprechend auf der .kollektiven Ebene modelliert werden sollte. Nimmt man den Prädiktor auf beiden Ebenen ins Modell auf, erweist sich der Effekt auf L2 häufig erwartungswidrig als nicht-signifikant, was jedoch zu inhaltlich falschen Schlüssen führen kann, da die doppelte Aufnahme zu einer Art „over controlling “ führen kann.

Während die Mehrebenenanalyse zu Beginn ihrer Entwicklung nur als spezialisierte StatistikSoftware verfügbar war, gehört sie heute auch zum normalen Funktionsumfang von StatistikPaketen wie SAS, SPSS und STATA und R. Jedoch werden die Spezialprogramme HLM und MLWin heute immer noch sehr häufig verwendet, daneben benutzen viele Forscher SAS und STATA, für das es auch das besonders umfangreiche und leistungsstarke Zusatzmodul GLLAMM gibt (Rabe-Hesketh/Skrondal 2012). Als Besonderheit bietet MPlus (Muthen/Muthen 2012) eine Verbindung von Mehrebenenanalysen mit Strukturgleichungsmodellen, wodurch Kontexteffekte als latente Variablen modelliert und auch Pfadanalysen auf mehreren Ebenen durchgeführt werden können.

\section{Ausblick}

Die Auswirkungen sozialräumlicher Konzentrationen von Armut und anderen Problemlagen auf Kriminalität sind nicht nur ein „klassisches“ Thema der Kriminalsoziologie, sondern auch ein sehr lebendiges Forschungsgebiet mit hohen Wachstumsraten in den vergangenen Jahren. Die theoretische und methodische Weiterentwicklung dieses Feldes hat dazu beigetragen, dass wir heute erheblich mehr über kollektive Einflüsse des Wohnquartiers auf das delinquente Verhalten von Jugendlichen und auf die Unsicherheitswahrnehmungen der Bewohner wissen als noch vor zehn bis fünfzehn Jahren. Diese Forschung spielt sich jedoch noch ganz überwie- gend außerhalb Deutschlands, nämlich in den USA und einigen europäischen Ländern wie Großbritannien und den Niederlanden ab, und bei der Übertragbarkeit der Ergebnisse sollten nationale Unterschiede in den gesellschaftlichen - insbesondere wohlfahrtsstaatlichen - Rahmenbedingungen berücksichtigt werden. Wenn sich beispielsweise die Auswirkungen von Disorder-Erscheinungen auf das Sicherheitsgefühl und das kollektive Sozialkapital in urbanen Räumen, die sich in U.S.-amerikanischen Studien gezeigt haben, grundsätzlich auch für Deutschland bestätigen lassen, so ersetzt dies nicht eigene, methodisch angemessene Forschungen, von der es in Deutschland bislang zu wenig gegeben hat. Das gilt insbesondere für Längsschnittsstudien, die in ihrer Aussagekraft über Querschnittsstudien hinausgehen. 
betrifft, so erscheinen ihre Wirkungen im Vergleich zu individuellen Risikofaktoren als eher schwach, zumal im Kontext europäischer Wohlfahrtsstaaten. Das bedeutet jedoch nicht, dass sie vernachlässigbar sind. Es ist nämlich auch deutlich geworden, dass die Suche nach ,durchschnittlichen“" Kontexteffekten wenig sinnvoll ist, sondern dass es entscheidend darauf ankommt, welche Wirkungen sozialräumliche Problemlagen auf besonders vulnerable Kinder und Jugendliche ausüben, und welche wechselseitigen Verstärkungen von indivi-

duellen und kollektiven Risiken dabei auftreten können. Um diese auf den ersten Blick schwer zu erkennenden Effekte differenziert zu erfassen, ist eine enge Verzahnung der sozialökologischen Perspektive mit anderen soziologischen und psychologischen Forschungsansätzen notwendig. Nur durch eine theoriegeleitete Forschung, die sich bemüht, die sozialen Mechanismen der Wirkung sozialstruktureller Benachteiligungen auf Einstellungen und Verhalten von Jugendli- chen aufzuklären, kann sich dieses Forschungsfeld produktiv weiter entwickeln. Dabei spielen methodische Innovationen wie die Mehrebenenanalyse, aber auch die Kombination unterschiedlicher und teils neuer Erhebungsverfahren wie die Messung von Aktionsräumen und Netzwerkanalysen eine wichtige Rolle. Während sich bei der Analyse der Problemlagen zumindest in Ansätzen ein gesichertes Wissen abzeichnet, gilt dies für die Frage, welche Interventionen gegen negative Verstärkungseffekten durch sozialräumliche Benachteiligungen sinnvoll und möglich sind, keinesfalls. Experimentelle Interventionsstudien sind in diesem Bereich extrem selten und haben unerwartete und widersprüchliche Ergebnisse erbracht. Bei dem umfangreichen und teuren städtebaulichen Programm „Soziale Stadt“ wurde auf eine aussagekräftige Wirkungsevaluation verzichtet. Auch die Wirkungen der aufwändigsten staat- lichen Intervention in das Leben von Kindern und Jugendlichen, der Schule, auf die Folgen sozialräumlicher Benachteiligungen, ist bislang unzureichend erforscht.

\section{Literatur}

Agnew, R., 1999. A general strain theory of community differences in crime rates. Journal of Research in Crime and Delinquency, 36(2), 123-155.

Akers, R.L., Jensen, G.F., 2006. The empirical status of social learning theory of crime and deviance: the past, present, and future. In: Cullen F.T., Wright J.P., Blevins K.R. (Hg.), Taking stock. The status of criminological theory. Roxbury: Transaction Publishers, 37- 
76.

Anderson, E., 1999. Code of the street: decency, violence, and the moral life of the inner city. New York: W. W. Norton.

Andrews, R., 2009. Civic engagement, ethnic heterogeneity, and social capital in urban areas: evidence from England. Urban Affairs Review, 44, 428-440.

Anselin, L., 2002. Spatial effects and ecological inference. Political Analysis, 10(3), 276-297.

Arum, R., 2000. Schools and communities: ecological and institutional dimensions. Annual Review of Sociology, 26, 395-418.

Baier, D., Rabold, S., 2009. Jugendgewalt in segregierten Stadtteilen. Deutsche Zeitschrift für Kommunalwissenschaften,(2), 35-49.

Baumer, E.P., Arnio, A.N., 2012. Multi-level modeling and criminological inquiry. In: Gadd,

D., Karstedt, S., Messner, S. (Hg.), The Sage handbook of criminological research methods. Los Angeles: Sage, 97-111.

Baur, C., Häußermann, H., 2009. Ethnische Segregation in deutschen Schulen. Leviathan, 37, 353-366.

Beaver, K.M., Wright, J.P., Maume, M.O., 2008. The effect of school classroom characteristics on low self-control: A multilevel analysis. Journal of Criminal Justice, 36(2), 174181.

Beaver, K.M., Gibson, C.L., DeLisi, M., Vaughn, M.G., Wright, J.P., 2012. The interaction between neighborhood disadvantage and genetic factors in the prediction of antisocial outcomes. Youth Violence and Juvenile Justice, 10(1), 25-40.

Belina, B., Wehrheim, J., 2011. "Gefahrengebiete" - Durch die Abstraktion vom Sozialen zur Reproduktion gesellschaftlicher Strukturen. In: Oberwittler, D., Behr, R. (Hg.), Polizei und Polizieren in multiethnischen Gesellschaften. Freiburg: Centaurus, 207-230.

Bellair, P.E., 1997. Social interaction and community crime: Examining the importance of neighbor networks. Criminology, 35, 677-703.

Bellair, P.E., 2000. Informal surveillance and street crime: a complex relationship. Criminology, 38(1), 137-169.

Bellair, P.E., Browning, C.R., 2010. Contemporary disorganization research: an assessment and further test of the systemic model of neighborhood crime. Journal of Research in Crime and Delinquency, 47(4), 496-521. 
Bellair, P.E., McNulty, T.L., 2005. Beyond the bell curve: community disadvantage and the explanation of black-white differences in adolescent violence. Criminology, 43(4), 1135 1167.

Bernasco, W., 2010. Modeling micro-level crime location choice: application of the discrete choice framework to crime at places. Journal of Quantitative Criminology, 26, 113-138.

Bernasco, W., Elffers, H., 2009. Statistical analysis of spatial crime Data. In: Piquero A.R., Weisburd D. (Hg.), Handbook of quantitative criminology. New York: Springer.

Bernburg, J.G., Thorlindsson, T., 2005. Violent values, conduct norms, and youth aggression: a multilevel study in Iceland. Sociological Quarterly, 46, 457-478.

Bernburg, J.G., Thorlindsson, T., 2007. Community structure and adolescent delinquency in Iceland: A contextual analysis. Criminology, 45(2), 415-444.

Bernburg, J.G., Thorlindsson, T., Sigfusdottir, I.D., 2009. Relative deprivation and adolescent outcomes in iceland: a multilevel test. Social Forces, 87(3), 1223-1250.

Beyers, J.M., Bates, J.E., Pettit, G.S., Dodge, K.A., 2003. Neighborhood structure, parenting processes, and the development of youths externalizing behaviors: a multilevel analysis. American Journal of Community Psychology, 31(1/2), 35-53.

Bingenheimer, J.B., Brennan, R.T., Earls, F.J., 2005. Firearm violence exposure and serious violent behavior. Science, 308, 1323-1326.

Booth, A., Crouter, A.C. (Hg.), 2001. Does it take a village? Community effects on children, adolescents, and families. Mahwah, NJ: Erlbaum.

Bottoms, A.E., 2007. Place, space, crime and disorder. In: Maguire, M., Morgan, R., Reiner, R. (Hg.), The Oxford handbook of criminology (4. A.). Oxford: Oxford University Press, 528-574.

Bottoms, A.E., 2012. Developing socio-spatial criminology. In: Maguire M., Morgan R., Reiner R. (Hg.), The Oxford handbook of criminology (5. A.). Oxford: Oxford University Press, 450-488.

Boudon, R., 1998. Social mechanisms without black boxes. In: Hedström, P., Swedberg, R. (Hg.), Social mechanisms. an analytical approach to social theory. Cambridge: Cambridge University Press, 172-203.

Bowers, K.J., Johnson, S.D., 2005. Domestic burglary repeats and space time clusters the dimensions of risk. European Journal of Criminology, 2(1), 67-92.

Bradshaw, C., Sawyer, A.L., O'Brennan, L.M., 2009. A social disorganization perspective on bullying-related attitudes and behaviors: the influence of school context. American Journal of Community Psychology, 43, 204-220.

Brännström, L., 2004. Poor places, poor prospects? counterfactual models of neighbourhood 
effects on social exclusion in Stockholm, Sweden. Urban Studies, 41(13), 2515-2537.

Brännström, L., 2006. Phantom of the neighbourhood. longitudinal studies on area-based conditions and individual outcomes. Stockholm: Stockholm University.

Brännström, L., 2008. Making their mark: the effects of neighbourhood and upper secondary school on educational achievement. European Sociological Review, 24(4), 463-478.

Brännström, L., Rojas, Y., 2012. Rethinking the long-term consequences of growing up in a

Braga, A.A., 2005. Hot spots policing and crime prevention: A systematic review of randomized controlled trials. Journal of Experimental Criminology, 1, 317-342.

Brantingham, P.J., Brantingham, P.L., 2008. Crime pattern theory. In: Wortley, R., Mazerolle, L. (Hg.), Environmental Criminology and Crime Analysis. Cullompton: Willan, 78-93.

Brody, G.H., Ge, X., Conger, R.D., Gibbons, F.X., McBride Murry, V., Gerrard, M., Simons, R.L., 2001. The influence of neighborhood disadvantage, collective socialization, and parenting on African American children's affiliation with deviant Peers. Child Development, 72(4), 1231-1246.

Bronfenbrenner, U., 1979. The ecology of human development. Cambridge/Mass.: Harvard University Press.

Brookmeyer, K.A., Fanti, K.A., Henrich, C.C., 2006. Schools, parents, and youth violence: a multilevel, ecological analysis. Journal of Clinical Child and Adolescent Psychology, $35(4), 504-514$.

Browning, C.R., Feinberg, S.L., Dietz, R.D., 2004. The paradox of social organization: networks, collective efficacy, and violent crime in neighborhoods. Social Forces, 83(2), 503534.

Browning, C.R., Leventhal, T., Brooks-Gunn, J., 2005. Sexual initiation in early adolescence: the nexus of parental and community control. American Sociological Review, 70, 758-778.

Browning, C.R., Burrington, L.A., Leventhal, T., Brooks-Gunn, J., 2008. Neighborhood structural inequality, collective efficacy, and sexual risk behavior among urban youth. Journal of Health and Social Behavior, 49(3), 269-285.

Brunton-Smith, I., 2011. Untangling the relationship between fear of crime and perceptions of disorder. evidence from a longitudinal study of young people in England and Wales. Brit- 
ish Journal of Criminology, 51, 885-899.

Brunton-Smith, I., Sturgis, P., 2011. Do neighborhoods generate fear of crime? An empirical test using the British crime survey. Criminology, 49(2), 331-369.

Burchfield, K.B., 2009. Attachment as a source of informal social control in urban neighborhoods. Journal of Criminal Justice, 37, 45-54.

Bursik, R.J.J., Grasmick, H.G., 1993. Neighborhoods and crime: the dimensions of effective

Bursik, R.J.J., 1999. The informal control of crime through neighborhood networks. Sociological Focus, 32, 85-97.

Carr, P., 2003. The new parochialism: the implications of the beltway case for arguments concerning informal social control. American Journal of Sociology, 108(6), 1249-1291.

Carr, P.J., 2005. Clean streets: crime, disorder, and social control in a Chicago neighborhood. New York: New York University Press.

Caspi, A., McClay, J., Moffitt, T.E., Mill, J., Craig, I.W., Taylor, A., Poulton, R., 2002. The role of genotype in the cycle of violence in maltreated children. Science, 297(2 August), 851-854.

Ceccato, V., Oberwittler, D., 2008. Comparing spatial patterns of robbery: evidence from a western and an eastern European city. Cities, 25(4), 185-196.

Clampet-Lundquist, S., Edin, K., Kling, J.R., Duncan, G.J., 2011. Moving teenagers out of high-risk neighborhoods: how girls fare better than boys. American Journal of Sociology, 116(4), 1154-1189.

Clark, J.P., Wenninger, E.P., 1962. Socio-economic class and area as correlates of illegal behaviour among juveniles. American Sociological Review, 27, 826-834.

Clarke, R.V., 2009. Situational crime prevention: theoretical background and current practice. In: Krohn, M.D., Lizotte, A.J., Hall, G.P. (Hg.), Handbook on crime and deviance. Dordrecht: Springer, 259-276.

Coleman, J., 1988. Social capital in the creation of human capital. American Journal of Sociology, 94, 95-120.

Coleman, J.S., 1961. Community disorganization. In: Merton, R.K., Nisbet, R.A. (Hg.), Contemporary social problems. An introduction to the sociology of deviant behavior and social disorganization. New York: Harcourt, Brace \& World, 553-604.

Cornish, D.A., Clarke, R.V. (Hg.), 1986. The Reasoning criminal: rational choice perspectives on offending. New York: Springer.

Covington, J., Taylor, R.B., 1991. Fear of crime in urban residential neighborhoods: implications of between and within-neighborhood sources for current models. The Sociological 
Quarterly,(32), 231-249.

Crane, J., 1991. The epidemic theory of ghettos and neighborhood effects on dropping out and

Crowder, K.D., South, S.J., 2003. Neighborhood distress and school dropout: the variable significance of community context. Social Science Research, 32(4), 659-698.

Cullen, J.B., Levitt, S.D., 1999. Crime, urban flight, and the consequences for cities. The Review of Economics \& Statistics, 81(2), 159-169.

De Coster, S., Heimer, K., Wittrock, S.M., 2006. Neighborhood disadvantage, social capital, street context, and youth crime. Sociological Quarterly, 47(4), 723-753.

de Leeuw, J., Meijer, E. (Hg.), 2008. Handbook of multilevel analysis. New York: Springer.

Dehejia, R.H., Wahba, S., 2002. Ppropensity score-matching methods for nonexperimental causal studies. The Review of Economics \& Statistics, 84(1), 151-161.

DeLuca, S., Dayton, E., 2009. Switching social contexts: the effects of housing mobility and school choice programs on youth outcomes. Annual Review of Sociology, 35, 457-491.

Diez Roux, A.V., 2004. Estimating neighborhood health effects: the challenges of causal inference in a complex world. Social Science \& Medicine, 58, 1953-1960.

Diez Roux, A.V., Mair, C., 2010. Neighborhoods and health. Annals of the New York Academy of Sciences, 1186, 125-145.

DiPrete, T.A., Forristal, J.D., 1994. Multilevel models: methods and substance. Annual Review of Sociology, 20, 331-357.

Ditton, H., Krüsken, J., 2006. Sozialer Kontext und schulische Leistungen - zur Bildungsrelevanz segregierter Armut. Zeitschrift für Soziologie der Erziehung und Sozialisation, 26(2), 135-157.

Ditton, H., 2009. Familie und Schule - eine Bestandsaufnahme der bildungssoziologischen Schuleffektsforschung von James S. Coleman bis heute. In: Becker, R. (Hg.), Lehrbuch der Bildungssoziologie. Wiesbaden: VS Verlag für Sozialwissenschaften, 239-258.

Dodge, K.A., Pettit, G.S., 2003. A Biopsychosocial model of the development of chronic conduct problems in adolescence. Developmental Psychology, 39(2), 349-371.

Duncan, G.J., Raudenbush, S.W., 1999. Assessing the effect of context in studies of child and youth development. Educational Psychologist, 34(1), 29-41. 
Duncan, G.J., Raudenbush, S.W., 2001. Neighborhoods and adolescent development: how can we determine the links? In: Booth, A., Crouter, A.C. (Hg.), Does it Take a Village? Community Effects on Children, Adolescents, and Families. Mahwah, NJ: Erlbaum, 105-136.

Duncan, G.J., Magnuson, K.A., Ludwig, J., 2004. The endogeneity problem in developmental studies. Research in Human Development, 1(1\&2), 59-80.

Duncan, G.J., Murnane, R. (Hg.), 2011. Whither opportunity? Rising inequality, schools, and children's life chances. New York: Russell Sage Foundation.

Dupéré, Véronique, Lacourse, É., Willms, D., Leventhal, T., Tremblay, R.E., 2008. Neighborhood poverty and early transition to sexual activity in young adolescents: a developmental ecological approach. Child Development, 79(5), 1463-1476.

Eifler, S., Thume, D., Schnell, R., 2009. Unterschiede zwischen subjektiven und objektiven Messungen von Zeichen öffentlicher Unordnung ("Signs of incivility"). In: Weichbold, M., Bacher, J., Wolf, C. (Hg.), Umfrageforschung. Herausforderungen und Grenzen. Wiesbaden: VS Verlag für Sozialwissenschaften, 415-442.

Eisner, M., 1997. Das Ende der zivilisierten Stadt? Die Auswirkungen von Modernisierung und urbaner Krise auf Gewaltdelinquenz. Frankfurt am Main: Campus.

Elliott, D.S., Menard, S., Rankin, B.H., Wilson, W.J., Huizinga, D., 2006. Good kids from bad neighborhoods. successful development in social context. Cambridge: Cambridge University Press.

Elwert, F., Winship, C. im Druck. Endogenous selection bias. Annual Review of Sociology.

Enders, C.K., Tofighi, D., 2007. Centering predictor variables in cross-sectional multilevel models: a new look at an old issue. Psychological Methods, 12(2), 121-138.

Esser, H., 1996. Soziologie. Allgemeine Grundlagen. Frankfurt am Main: Campus.

Fagan, A.A., Wright, E.M., 2012. The effects of neighborhood context on youth violence and delinquency. does gender matter? Youth Violence and Juvenile Justice, 10(1), 64-82.

Farrall, S., Jackson, J., Gray, E., 2009. Social order and the fear of crime in contemporary times. Oxford: Oxford University Press.

Farwick, A., 2012. Segregation. In: Eckardt, F. (Hg.), Handbuch Stadtsoziologie. Wiesbaden: Springer VS, 381-419.

Fauth Rebecca C., Roth Jodie L., Brooks-Gunn, J., 2007. Does the neighborhood context alter the link between youth's after-school time activities and developmental outcomes? A multilevel analysis. Developmental Psychology, 43(3), 760-777. 
Fincke, G., Lange, S., 2012. Segregation an Grundschulen: Der Einfluss der elterlichen

Schulwahl Sachverständigenrat deutscher Stiftungen für Integration und Migration. (Internet-Publikation, www.svr-migration.de/content, abgerufen am 4.3.2013).

Frehsee, D., 1979. Strukturbedingungen urbaner Kriminalität: Eine Kriminalgeographie der Stadt Kiel unter besonderer Berücksichtigung der Jugendkriminalität. Göttingen: Otto Schwarz.

Friedrichs, J., 1988. Makro- und Mikrosoziologische Theorien der Segregation. In: Friedrichs, J. (Hg.), Soziologische Stadtforschung. Opladen: Westdeutscher Verlag, 56-77.

Friedrichs, J., 1997. Normenpluralität und abweichendes Verhalten: Eine theoretische und empirische Analyse. In: Heitmeyer, W. (Hg.), Was treibt die Gesellschaft auseinander? Frankfurt am Main: Suhrkamp, 473-505.

Friedrichs, J., 1998. Do poor neighborhoods make their residents poorer? Context effects of poverty neighborhoods on residents. In: Andreß, H.-J. (Hg.), Empirical poverty research in a comparative perspective. Aldershot: Ashgate, 77-98.

Friedrichs, J., Blasius, J., 2000. Leben in benachteiligten Wohngebieten. Opladen: Leske + Budrich.

Friedrichs, J., Galster, G., Musterd, S., 2003. Neighbourhood effects on social opportunities: the European and American research and policy context. Housing Studies, 18(6), 797-806.

Friedrichs, J., Oberwittler, D., 2007. Soziales Kapital in Wohngebieten. In: Franzen, A., Freitag, M. (Hg.), Sozialkapital. Wiesbaden: VS Verlag für Sozialwissenschaften, 450-486.

Fuchs, M., Schmalz, S., 2010. Gewalt an Schulen - eine Mehrebenenanalyse zum Einfluss von Sozialisationsbedingungen und Klassenkomposition. Zeitschrift für Soziologie der Erziehung und Sozialisation, 30(2), 134-148.

Furstenberg, F.F.J., Cook, T.D., Eccles, J., Elder, G.H.J., Sameroff, A., 1999. Managing to make it. urban families and adolescent success. Chicago: University of Chicago Press.

Garcia, R.M., Taylor, R.B., Lawton, B.A., 2007. Impacts of violent crime and neighborhood structure on trusting your neighbors. Justice Quarterly, 24(4), 679-704.

Gau, J.M., Pratt, T.C., 2008. Broken windows or window dressing? Citizens (in)ability to tell the difference between disorder and crime. Criminology \& Public Policy, 7(2), 163-194.

Gault, M., Silver, E., 2008. Spuriousness or mediation? Broken windows according to Sampson and Raudenbush (1999). Journal of Criminal Justice, 36, 240-243. 
Gibson, C.L., Zhao, J., Lovrich, N.P., Gaffney, M.J., 2002. Social integration, individual perceptions of collective efficacy, and fear of crime in three cities. Justice Quarterly, 19(3), 537-564.

Goldsmith, P.R., 2009. Schools or neighborhoods or both? Race and ethnic segregation and educational attainment. Social Forces, 87(4), 1913-1942.

Gottfredson, G.D., Gottfredson, D.C., Payne, A.A., Gottfredson, N.C., 2005. School climate predictors of school disorder: results from a national study of delinquency prevention in schools. Journal of Research in Crime and Delinquency, 42(4), 412-444.

Gottfredson, M.R., Hirschi, T., 1990. General Theory of Crime. Stanford: Stanford University Press.

Goudriaan, H., Wittebrood, K., Nieuwbeerta, P., 2006. Neighborhood characteristics and reporting crime. Effects of social cohesion, confidence in police effectiveness ans socio-economic disadvantage. British Journal of Criminology, 46, 719-742.

Granovetter, M., 1973. The strength of weak ties. American Journal of Sociology, 78(6), 1360-1380.

Greve, J., Schnabel, A., Schützeichel, R. (Hg.), 2008. Das Mikro-Makro-Modell der soziologischen Erklärung. Wiesbaden: VS Verlag für Sozialwissenschaften.

Guo, G., Roettger, M.E., Cai, T., 2008. The integration of genetic propensities into socialcontrol models of delinquency and violence among male youths. American Sociological Review, 73(4), 543-568.

Häußermann, H., 2008. Wohnen und Quartier: Ursachen sozialräumlicher Segregation. In: Huster, E.-U., Boekh, J., Mogge-Grotjahn, H. (Hg.), Handbuch Armut und soziale Ausgrenzung. Wiesbaden: VS Verlag für Sozialwissenschaften, 335-349.

Harding, D.J., 2003. Counterfactual models of neighborhood effects: the effect of neighborhood poverty on dropping out and teenage pregnancy. American Journal of Sociology, 109(3), 676-719.

Harding, D.J., 2007. Cultural context, sexual behavior, and romantic relationships in disadvantaged neighborhoods. American Sociological Review, 72, 341-364.

Harding, D.J., 2008. Neighborhood violence and adolescent friendships. The International Journal of Conflict and Violence, 2(1), 28-55.

Harding, D.J., 2009. Violence, older peers, and the socialization of adolescent boys in disadvantaged neighborhoods. American Sociological Review, 74, 445-464.

Harding, D.J., 2009. Collateral consequences of violence in disadvantaged neighborhoods. 
Social Forces, 88(2), 757-784.

Harding, D.J., 2010. Living the drama. community, conflict, and culture among inner-city boys. Chicago: Chicago University Press.

Harding, D.J., Gennetian, L., Winship, C., Sanbonmatsu, L., Kling, J.R., 2011. Unpacking neighborhood influences on education outcomes: setting the stage for future research. In: Duncan, G.J., Murnane, R. (Hg.), Whither opportunity? Rising inequality, schools, and children's life chances. New York: Russell Sage Foundation, 277-298.

Harris, K.M., Duncan, G.J., Boisjoly, J., 2002. Evaluating the role of "nothing to lose" attitudes on risky behavior in adolescence. Social Forces, 80(3), 1005-1039.

Hay, C., Fortson, E.N., Hollist, D.R., Altheimer, I., Schaible, L.M., 2007. Compounded risk: the implications for delinquency of coming from a poor family that lives in a poor community. Journal of Youth and Adolescence, 36, 593-605.

Haynie, D.L., Osgood, D.W., 2005. Reconsidering peers and delinquency: How do peers matter? Social Forces, 84(2), 1-34.

Haynie, D.L., Silver, E., Teasdale, B., 2006. Neighborhood characteristics, peer networks, and adolescent violence. Journal of Quantitative Criminology,(22), 147-169.

Hedström, P., Swedberg, R., 1998. Social mechanisms: An introductory essay. In: Hedström, P., Swedberg, R. (Hg.), Social mechanisms. an analytical approach to social theory. Cambridge: Cambridge University Press, 1-32.

Heitmeyer, W., Thome, H., Kock, S., Marth, J., Thöle, U., Schroth, A., van de Wetering, D. (Hg.), 2011. Gewalt in öffentlichen Räumen. Zum Einfluss von Bevölkerungs- und Siedlungsstrukturen in städtischen Wohnquartieren. Wiesbaden: VS Verlag für Sozialwissenschaften.

Helbig, M., 2010. Neighborhood does matter! Soziostrukturelle Nachbarschaftscharakteristika und Bildungserfolg. Kölner Zeitschrift für Soziologie und Sozialpsychologie, 62(4), 655679.

Hipp, J.R., Perrin, A., 2006. Nested loyalties: local networks' effects on neighbourhood and community cohesion. Urban Studies, 43, 2503-2535.

Hirschi, T., 1969. Causes of delinquency. Berkeley: University of California Press.

Hirtenlehner, H., 2006. Kriminalitätsfurcht - Ausdruck generalisierter Ängste oder schwindener Gewissheiten? Kölner Zeitschrift für Soziologie und Sozialpsychologie, 58(2), 307331.

Hirtenlehner, H., 2008. Disorder, social anxieties and fear of crime. exploring the relationship between incivilities and fear of crime with a special focus on generalized insecurities. In: 
Kury, H. (Hg.), Fear of crime - punitivity. new developments in theory and research. Bochum: Universitätsverlag Brockmeyer, 127-158.

Holm, A., 2010. Gentrifizierung und Kultur: Zur Logik kulturell vermittelter Aufwertungsprozesse. In: Hannemann, C., Glasauer, H., Pohlan, J., Pott, A., Kirchberg ,V. (Hg.), Jahrbuch StadtRegion 2009/2010. Schwerpunkt: Stadtkultur und Kreativität. Opladen: Verlag Barbara Budrich, 64-82.

Holm, A., 2012. Gentrification. In: Eckardt, F. (Hg.), Handbuch Stadtsoziologie. Wiesbaden: Springer VS, 661-687.

Hox, J., 2010. Multilevel analysis. techniques and applications (2. A.). New York: Routledge.

Hox, J., Roberts, J.K. (Hg.), 2011. The handbook of advanced multilevel analysis. New York: Routledge.

Hox, J., van de Schoot, R., Matthijsse, S., 2012. How few countries will do? Comparative survey analysis from a Bayesian perspective. Survey Research Methods, 6(2), 87-93.

Hummelsheim, Dina, Hirtenlehner, H., Jackson, J., Oberwittler, D., 2011. Social insecurities and fear of crime: a cross-national study on the impact of welfare state policies on crimerelated anxieties. European Sociological Review, 27(3), 327-345.

Hurrelmann, K., 1983. Das Modell des produktiv realitätsverarbeitenden Subjekts in der Sozialisationsforschung. Zeitschrift für Sozialisationsforschung und Erziehungssoziologie, 3(1), 91-104.

Ingoldsby, E.M., Shaw, D.E., Winslow, E., Schonberg, M.A., Gilliom, M., Criss, M.M., 2006. Neighborhood disadvantage, parent-child conflict, neighborhood peer relationships, and early antisocial behavior problem trajectories. Journal of Abnormal Child Psychology, 34(3), 303-319.

Innes, M., 2004. Signal crimes and signal disorders: notes on deviance as communicative action. British Journal of Sociology, 55(3), 335-355.

Jackson, J., 2004. Experience and expression. social and cultural significance in the fear of crime. British Journal of Criminology, 44(6), 946-966.

Jackson, M.I., Mare, R.D., 2007. Cross-Sectional and longitudinal measurements of neighbourhood experience and their effects on children. Social Science Research, 36(2), 590610. 
Jones, N., 2010. Between good and ghetto: African American girls and inner-city violence. New Brunswick: Rutgers University Press.

Karriker-Jaffe, K.J., Foshee, V.A., Ennett, S.T., Suchindran, C., 2009. Sex differences in the effects of neighborhood socioeconomic disadvantage and social organization on rural adolescents aggression trajectories. American Journal of Community Psychology, 43, 189203.

Kasarda, J., Janowitz, M., 1974. Community attachment in mass society. American Sociological Review, 39 (3), 328-339.

Kauppinen, T.M., 2008. Schools as mediators of neighbourhood effects on choice between vocational and academic tracks of secondary education in Helsinki. European Sociological Review, 24, 379-391.

Kirk, D.S., 2008. The neighborhood context of racial and ethnic disparities in arrest. Demography, 45(1), 55-77.

Kirk, D.S., 2009. Unraveling the contextual effects on student suspension and juvenile arrest: the independent and interdependent influences of school, neighborhood, and family social controls. Criminology, 47(2), 479-520.

Kirk, D.S., Laub, J.H., 2010. Neighborhood change and crime in the modern metropolis. In: Tonry, M. (Hg.), Crime and Justice. A Review of Research /39. Chicago: Chicago University Press, 441-502.

Kirk, D.S., Matsuda, M., 2011. Legal cynicism, collective efficacy, and the ecology of arrest. Criminology, 49(2), 443-472.

Kivivuori, J., 2011. Discovery of hidden crime. Self-report delinquency surveys in criminal policy context. Oxford: Oxford University Press.

Klein, M.W., Weerman, F.M., Thornberry, T.P., 2006. Street gang violence in Europe. European Journal of Criminology, 3(4), 413-437. 
Klein, M.W., Maxson, C.L., 2006. Street gang patterns and policies. Oxford: Oxford University Press.

Kling, J.R., Ludwig, J., Katz, L., 2005. Neighborhood effects on crime for female and male youth: evidence from a randomized housing voucher experiment. Quarterly Journal of Economics, 120(1), 87-130.

Kling, J.R., Liebman, J.B., Katz, L.F., 2007. Experimental analysis of neighborhood effects. Econometrica, 75(1), 83-119.

Kornhauser, R.R., 1978. Social sources of delinquency: an appraisal of analytic models. Chicago:

Kreager, D.A., 2007. When it's good to be "bad": violence and adolescent peer acceptance. Criminology, 45(4), 893-923.

Kristen, C., 2008. Primary school choice and ethnic school segregation in German elementary schools. European Sociological Review, 24(4), 495-510.

Krohn, M.D., Thornberry, T.P., Gibson, C.L., Baldwin, J.M., 2010. The development and impact of self-report measures of crime and delinquency. Journal of Quantitative Criminology, 26, 509-525.

Kubrin, C.E., 2009. Social disorganization theory: Then, now and in the future. In: Krohn, M.D., Lizotte, A.J., Hall, G.P. (Hg.), Handbook on Crime and Deviance. Dordrecht et al.: Springer, 225-240.

Kunadt, S., 2011. Sozialer Raum und Jugendkriminalität. Zum Einfluss der Wohnumgebung auf delinquentes Handeln, eine empirische Untersuchung in Duisburg. Münster: Waxmann.

Lahey, B.B., Van Hulle, C.A., Onofrio, B.M., Lee Rodgers, J., Waldman, I.D., 2008. Is parental knowledge of their adolescent offspring's whereabouts and peer associations spuriously associated with offspring delinquency? Journal of Abnormal Child Psychology, 36, 807823.

Lange, A., 2008. Agency - eine Perspektive für die Jugendforschung. In: Homfeldt, H.G., Schröer, W., Schweppe, C. (Hg.), Vom Adressaten zum Akteur. Soziale Arbeit und Agency. Opladen: Verlag Barbara Budrich, 155-179.

Langer, W., 2004. Mehrebenenanalyse. Eine Einführung in Forschung und Praxis. Wiesbaden: VS Verlag für Sozialwissenschaften. 
Laurence, J., 2011. The effect of ethnic diversity and community disadvantage on social cohesion: a multi-level analysis of social capital and interethnic relations in UK communities. European Sociological Review, 27(1), 70-89.

Leckie, G., 2009. The complexity of school and neighbourhood effects and movements of pupils on school differences in models of educational achievement. Journal of the Royal Statistical Society: Series A, 172(3), 537-554.

Leventhal, T., Brooks-Gunn, J., 2000. The neighborhoods they live in: the effects of neighborhood residence on child and adolescent outcomes. Psychological Bulletin, 126, 309337.

Lo, C.C., Kim, Y.S., Allen, T.M., Allen, A.N., Minugh, P.A., Lomuto, N., 2011. The impact of school environment and grade level on student delinquency: a multilevel modeling approach. Crime \& Delinquency, 57(4), 622-657.

Loeber, R., Farrington, D.P. (Hg.), 1998. Serious and violent juvenile offenders: risk factors and successful interventions. Thousand Oaks, CA: Sage.

Ludwig, J., Liebman, J.B., Kling, J.R., Duncan, G.J., Katz, L.F., Kessler, R.C., Sanbonmatsu, L., 2008. What can we learn about neighborhood effects from the moving to opportunity experiment? American Journal of Sociology, 114(1), 144-188.

Lüdemann, C., 2006. Soziales Kapital und soziale Kontrolle. Zu den Determinanten sozialer Kontrolle in Nachbarschaften. Kriminalistik, 60(3), 177-182.

Lüdemann, C., 2006. Kriminalitätsfurcht im urbanen Raum. Eine Mehrebenenanalyse zu individuellen und sozialräumlichen Determinanten von Kriminalitätsfurcht im städtischen Raum. Kölner Zeitschrift für Soziologie und Sozialpsychologie, 58, 285-306.

Lüdtke, O., Robitzsch, A., Köller, O., 2002. Statistische Artefakte bei Kontexteffekten in der pädagogisch-psychologischen Forschung. Zeitschrift für Pädagogische Psychologie, 16(3/4), 217-231.

Lüdtke, O., Marsh, H.W., Robitzsch, A., Trautwein, U., Asparouhov, T., Muthén, B., 2008. The multilevel latent covariate model: A new, more reliable approach to group-level effects in contextual studies.. Psychological Methods, 13(3), 203-229.

Lupton, R., Kneale, D., 2012. Theorising and measuring place in neighbourhood effects research: the example of teenage parenthood in England. In: van Ham, M., Manley, D., Bai- 
ley, N., Simpson, L., Maclennan ,D. (Hg.), Neighbourhood Effects Research: New Perspectives. Dordrecht; et al.: Springer, 121-145.

Luthar, S.S. (Hg.), 2003. Resilience and vulnerability. Adaption in the context of childhood adversities. Cambridge: Cambridge University Press.

Lynam, D.R., Caspi, A., Moffitt, T.E., Wikström, P.-O.H., Loeber, R., Novak, S., 2000. The interaction between impulsivity and neighborhood context on offending: The effects of impulsivity are stronger in poorer neighborhoods. Journal of Abnormal Psychology, 109(4), 563-574.

Magnusson, D., Stattin, H., 2006. The person in the environment: towards a general model for scientific inquiry. In: Damon, W., Lerner, R.M. (Hg.), Handbook of child psychology, volume 1: theoretical models of human development (6. A.). New York: Wiley, 400-464.

Maimon, D., Browning, C.R., 2010. Unstructured socializing, collective efficacy, and violent behavior among urban youth. Criminology, 48(2), 443-474.

Maimon, D., Browning, C.R., 2012. Underage drinking, alcohol sales and collective efficacy: Informal control and opportunity in the study of alcohol use. Social Science Research, 41, 977-990.

Markowitz, F.E., Bellair, P.E., Liska, A.E., Liu, J., 2001. Extending social disorganization theory: modeling the relationships between cohesion, disorder, and fear. Criminology, 39(2), 293-319.

Marsh, H.W., Trautwein, U., Lüdtke, O., Baumert, J., Köller, O., 2007. The big-fish-littlepond effect: persistent negative effects of selective high schools on self-concept after graduation. American Educational Research Journal, 44(3), 631-669.

Marsh, H.W., Lüdtke, O., Robitzsch, A., Trautwein, U., Asparouhov, T., Muthén, B., Nagengast, B., 2009. Doubly-latent models of school contextual effects: integrating multilevel and structural equation approaches to control measurement and sampling error multivariate behavioral research. Multivariate Behavioral Research, 44(6), 764-802.

Massey, D.S., 1996. The age of extremes: concentrated affluence and poverty in the twentyfirst century. Demography, 33(4), 395-412.

Mazerolle, L.G., Wickes, R., McBroom, J., 2010. Community variations in violence: the role of social ties and collective efficacy in comparative context. Journal of Research in Crime and Delinquency, 47(1), 3-30. 
McNulty, T.L., Bellair, P.E., 2003. Explaining racial and ethnic differences in serious adolescent violent behavior. Criminology, 41(3), 709-748.

Meier, M.H., Slutske, W.S., Arndt, S., Cadoret, R.J., 2008. Impulsive and callous traits are more strongly associated with delinquent behavior in higher risk neighborhoods among boys and girls. Journal of Abnormal Psychology, 117(2), 377-385.

Molnar, B.E., Cerda, M., Roberts, A.L., Buka, S.L., 2008. Effects of neighborhood resources on aggressive and delinquent behaviors among urban youths. American Journal of Public Health, 98, 1086-1093.

Morenoff, J.D., Sampson, R.J., 1997. Violent crime and the spatial dynamics of neighborhood transition: Chicago, 1970-1990. Social Forces, 76 (1), 31-64.

Murray, D.M., Varnell, S.P., Blitstein, J.L., 2004. Design and analysis of group-randomized trials: a review of recent methodological developments. American Journal of Public Health, 94(3), 423.

Muthén, L., Muthén, B., 2012. Mplus user's guide. seventh edition.. Los Angeles: Muthén \& Muthén.

Ness, C.N., 2004. Why girls fight. Female youth violence in the inner city. Annales of the American Academy of Political and Social Science, 595(Sept), 32-48.

Nieuwbeerta, P., McCall, P.L., Elffers, H., Wittebrood, K., 2008. Neighborhood characteristics and individual homicide risks effects of social cohesion, confidence in the police, and socioeconomic disadvantage. Homicide Studies, 12(1), 90-116.

Oberwittler, D., 2003a. Geschlecht, Ethnizität und sozialräumliche Benachteiligung - überraschende Interaktionen bei sozialen Bedingungsfaktoren von Gewalt und schwerer Eigentumsdelinquenz von Jugendlichen. In: Lamnek, S., Boatca, M. (Hg.), Geschlecht - Gewalt - Gesellschaft. Opladen: Leske + Budrich, 269-294.

Oberwittler, D., 2003b. Die Messung und Qualitätskontrolle kontextbezogener Befragungsdaten mithilfe der Mehrebenenanalyse - am Beispiel des Sozialkapitals von Stadtvierteln. ZA-Informationen, 53, 11-41.

Oberwittler, D., 2004a. A multilevel analysis of neighbourhood contextual effects on serious juvenile offending. the role of subcultural values and social disorganization. European Journal of Criminology, 1(2), 201-235. 
Oberwittler, D., 2004b. Stadtstruktur, Freundeskreise und Delinquenz. Eine Mehrebenenanalyse zu sozialökologischen Kontexteffekten auf schwere Jugenddelinquenz. In: Oberwittler, D., Karstedt, S. (Hg.), Soziologie der Kriminalität. Wiesbaden: VS Verlag für Sozialwissenschaften, 135-170.

Oberwittler, D., 2007a. The effects of ethnic and social segregation on children and adolescents: recent research and results from a German multilevel study (Discussion Paper $\mathrm{Nr}$ SP IV 2007-603). Berlin: Wissenschaftszentrum Berlin für Sozialforschung, Arbeitsstelle Interkulturelle Konflikte und gesellschaftliche Integration.

Oberwittler, D., 2007b. The effects of neighbourhood poverty on adolescent problem behaviours: a multi-level analysis differentiated by gender and ethnicity. Housing Studies, 22(5), 781-803.

Oberwittler, D., 2008. Armut macht Angst. Ansätze einer sozialökologischen Interpretation der Kriminalitätsfurcht. In: Groenemeyer, A., Wieseler, S. (Hg.), Soziologie sozialer Probleme und sozialer Kontrolle. Realitäten, Repräsentationen und Politik. Festschrift für Günter Albrecht. Wiesbaden: VS Verlag für Sozialwissenschaften, 215-230.

Oberwittler, D., 2012. Delinquenz und Kriminalität als soziales Problem. In: Albrecht, G., Groenemeyer, A. (Hg.), Handbuch Soziale Probleme (2.A.). Wiesbaden: Springer VS, $772-860$.

Oberwittler, D., Gerstner, D., im Druck. Die Modellierung von Interaktionseffekten in Erklärungsmodellen selbstberichteter Delinquenz - Ein empirischer Vergleich von linearer OLS-Regression und negativer Binomialregression anhand der Wechselwirkungen von Risikoorientierungen und Scham. In: Eifer, S., Pollich, D. (Hg.), Empirische Forschung über Kriminalität. Perspektiven und Herausforderungen. Wiesbaden: Springer VS.

Oberwittler, D., Wikström, P.-O.H., 2009. Why small is better: advancing the study of the role of behavioral contexts in crime causation. In: Weisburd, D., Bernasco, W., Bruinsma, G. (Hg.), Putting Crime in its Place. Units of Analysis in Geographic Criminology. New York: Springer, 35-59.

Oberwittler, D., Wikström, P.-O.H., 2011. Ethnic mix and collective social capital in urban England A quantitative case study of Peterborough on a small area level. Präsentation bei der Konferenz "Diversity and Contact", Max Planck Institute for the Study of Religious and Ethnic Diversity, Göttingen, 29 April 2011. 
O'Brien Caughy, M., Hayslett-McCall, K.L., O'Campo, P.J., 2007. No neighborhood is an island: Incorporating distal neighborhood effects into multilevel studies of child developmental competence. Health \& Place, 13, 788-798.

Paccagnella, O., 2006. Centering or not centering in multilevel models? the role of the group mean and the assessment of group effects. Evaluation Review, 30, 66-85.

Patchin, J.W., Huebner, B.M., McCluskey, J.D., Varano, S.P., Bynum, T.S., 2006. Exposure to community violence and childhood delinquency. Crime \& Delinquency, 52(2), 307-332.

Pattillo-McCoy, M., 1999. Black picket fences: privilege and peril among the black middle class. Chicago: Chicago University Press.

Pauwels, L., 2007. Buurtinvloeden en jeugddelinquentie. Een toets van de Sociale Desorganisatietheorie. Den Haag: Boom Juridische Uitgevers.

Pauwels, L., Hardyns, W., 2009. Measuring community (dis)organizational processes through key informant analysis. European Journal of Criminology, 6(5), 401-417.

Pauwels, L., Hardyns, W., van der Velde, M., 2010. Social disorganisation, offending, fear and victimisation. Findings from Belgian studies on the urban context of crime. Den Haag: BJu Legal Publishers.

Payne, A.A., Gottfredson, D.C., Gottfredson, G.D., 2003. Schools as communities: the relationships among communal school organization, student bonding, and school disorder. Criminology, 41(3), 749-777.

Payne, A.A., 2008. A multilevel analysis of the relationships among communal school organization, student bonding, and delinquency. Journal of Research in Crime and Delinquency, 45(4), 429-455.

Perkins, D.D., Taylor, R.B., 1996. Ecological assessments of community disorder - their relationship to fear of crime and theoretical implications. American Journal of Community Psychology, 24(1), 63-107.

Pettit, B., 2004. Moving and children's social connections: neighborhood context and the consequences of moving for low-income families. Sociological Forum, 19(2), 285-311.

Pitts, J., 2008. Reluctant gangsters: the changing face of youth crime. Cullompton: Willan.

Portes, A., Vickstrom, E., 2011. Diversity, social capital, and cohesion. Annual Review of Sociology, 37(1), 461-479.

Pratt, T.C., Turner, M.G., Piquero, A.R., 2004. Parental socialization and community context: 
a longitudinal analysis of the structural sources of low self control. Journal of Research in Crime and Delinquency, 41(3), 219-243.

Pratt, T.C., Cullen, F.T., 2005. Assessing macro-level predictors and theories of crime: a meta-analysis. In: Tonry, M. (Hg.), Crime and Justice. An Annual Review of Research. Vol. 32. Chicago: Chicago University Press, 373-450.

Putnam, R.D., 2007. E pluribus unum: diversity and community in the twenty-first century the 2006 Johan Skytte prize lecture. Scandinavian Political Studies, 30(2), 137-174.

Rabe-Hesketh, S., Skrondal, A., 2012. Multilevel and longitudinal modeling using STATA (3. A.). College Station, TX: Stata Press.

Rankin, B.H., Quane, J.M., 2002. Social contexts and urban adolescent outcomes: the interrelated effects of neighborhoods, families, and peers on African-American youth. Social Problems, 49(1), 79-100.

Rasbash, J., Leckie, G., Pillinger, R., Jenkins, J., 2010. Children's educational progress: partitioning family, school and area effects. Journal of the Royal Statistical Society: Series A, 173(3), 657-682.

Raudenbush, S., Bryk, A., 2002. Hierarchical linear models: applications and data analysis methods (2.A.). Thousand Oaks, CA: Sage.

Raudenbush, S.W., Sampson, R.J., 1999. Ecometrics: toward a science of assessing ecological settings, with appliance to the systematic social observation of neighborhoods. Sociological Methodology, 29, 1-41.

Raudenbush, S.W., Sampson, R.J., 1999. Assessing direct and indirect effects in multilevel designs with latent variables. Sociological Methods \& Research, 28(2), 123-153.

Raudenbush, S.W., Johnson, C., Sampson, R.J., 2003. A multivariate, multilevel Rasch model for self-reported criminal behavior. Sociological Methodology, 33(1), 169-211.

Reckless, W.C., Dinitz, S., Murray, E., 1957. The 'good' boy in a high delinquency area. The Journal of Criminal Law, Criminology, and Police Science, 48(1), 18-25.

Reijneveld, S.A., Brugman, E., Verhulst, F.C., Verloove-Vanhorick, P., 2005. Area deprivation and child psychosocial problems. A national cross-sectional study among school-aged children. Social Psychiatry and Psychiatric Epidemiology, 40, 18-23.

Robinson, J.B., Lawton, B.A., Taylor, R.B., Perkins, D.D., 2003. Multilevel longitudinal impacts of incivilities: fear of crime, expected safety, and block satisfaction. Journal of

Quantitative Criminology, 19(3), 237-274.

Robitaille, É., Séguin, A.-M., Lacourse, É., Vitaro, F., Tremblay, R.E., 2011. Désavantage socioéconomique du quartier et comportements antisociaux des adolescents: Quelle est l'é- 
chelle spatiale la plus probante? Canadian Journal of Behavioural Science/Revue canadienne des sciences du comportement, 43(2), 78-88.

Ross, C., Jang, S.J., 1999. Neighborhood disorder, fear, and mistrust: the buffering role of social ties with neighbors. American Journal of Community Psychology, 28(4), 401-420.

Rovers, B., 1997. De Buurt een Broeinest? Een onderzoek naar de invloed van woonomgeving op jeugdcriminaliteit. Nijmegen: Ars Aequi Libri.

Rutter, M., 1979. Fifteen thousand hours: secondary schools and their effects on children. London: Open Books.

Rutter, M., Silberg, J., 2002. Gene-environment interplay in relation to emotional and behavioral disturbance. Annual Review of Psychology, 53, 463-490.

Sampson, R.J., 2004. Networks and neighbourhoods. the implications of connectivity for thinking about crime in the modern city. In: McCarthy, H., Miller, P., Skidmore, P. (Hg.), Network logic. who governs in an interconnected world? London: Demos, 157-166.

Sampson, R.J., 2006. How does community context matter? Social mechanisms and the explanation of crime rates. In: Wikström, P.-O.H., Sampson, R.J. (Hg.), Crime and its explanation: contexts, mechanisms and development. Cambridge: Cambridge University Press, 31-60.

Sampson, R.J., 2009. Disparity and diversity in the contemporary city: social (dis)order revisited. British Journal of Sociology, 60(1), 1-31.

Sampson, R.J., Morenoff, J.D., Earls, F., 1999. Beyond social capital: spatial dynamics of collective efficacy for children. American Sociological Review,(64), 633-660.

Sampson, R.J., Morenoff, J.D., Gannon-Rowley, T., 2002. Assessing "neighborhood effects": social processes and new directions in research. Annual Review of Sociology, 28, 443-478.

Sampson, R.J., Morenoff, J.D., Raudenbush, S.W., 2005. Social anatomy of racial and ethnic disparities in violence. American Journal of Public Health, 95(2), 224-232.

Sampson, R.J., Raudenbush, S.W., Earls, F.J., 1997. Neighborhoods and violent crime: a multilevel study of collective efficacy. Science, 277, 918-924.

Sampson, R.J., Raudenbush, S.W., 1999. Systematic social observation of public spaces: a 
new look at disorder in urban neighborhoods. American Journal of Sociology, 105(3), 603-651.

Sampson, R.J., Sharkey, P., Raudenbush, S.W., 2008. Durable effects of concentrated disadvantage on verbal ability among African-American children. Proceedings of the National Academy of Sciences, 105(3), 845-852.

Sampson, R.J., Wikström, P.-O.H., 2008. The social order of violence in Chicago and Stockholm neighborhoods: a comparative inquiry. Cambridge: Cambridge University Press, 97119.

Schonberg, M.A., Shaw, D.S., 2007. Do the predictors of child conduct problems vary by high- and low-levels of socioeconomic and neighborhood risk? Clinical Child and Family Psychology, 10(2), 101-136.

Schulz, S., Eifler, S., Baier, D., 2011. Wer Wind sät, wird Sturm ernten. Die Transmission von Gewalt im empirischen Theorienvergleich. Kölner Zeitschrift für Soziologie und Sozialpsychologie, 63, 111-145.

Sharkey, P., Elwert, F., 2011. The legacy of disadvantage: multigenerational neighborhood effects on cognitive ability. American Journal of Sociology, 116(6), 1934-1984.

Shaw, C., McKay, H.D., 1969 [1942]. Juvenile delinquency and urban areas. Chicago: Chicago University Press.

Sherman, L.W., Gartin, P.R., Buerger, M.E., 1989. Hot spots of predatory crime: routine activities and the criminology of place. Criminology, 27(1), 27-55.

Silver, E., Miller, L.L., 2004. Sources of informal social control in Chicago neighborhoods. Criminology, 42(3), 551-583.

Simons, R.L., Kit Lei, M., Beach, S.R.H., Brody, G.H., Philibert, R.A., Gibbons, F.X., 2011. Social environment, genes, and aggression: evidence supporting the differential susceptibility perspective. American Sociological Review, 76(6), 883-912.

Skogan, W.G., 1990. Disorder and decline. crime and the spiral of decay in American neighborhoods. New York: Free Press.

Skogan, W.G., 2008. Broken windows: why - and how - we should take them seriously, Criminology \& Public Policy 7(2), 401-408.

Small, M.L., Feldman, J., 2012. Ethnographic evidence, heterogeneity, and neighbourhood effects after moving to opportunity. In: van Ham, M., Manley, D., Bailey, N., Simpson, L., 
Maclennan, D. (Hg.), Neighbourhood effects research: new perspectives. Dordrecht; et al.: Springer, 57-77.

Snijders, T.A.B., Bosker, R.J., 2012. Multilevel analysis. an introduction to basic and advanced multilevel analysis (2. A.). London: Sage.

Sobel, M.E., 1995. Causal inference in the social and behavioral sciences. In: Arminger, G., Clogg, C.C., Sobel, M.E. (Hg.), Handbook of statistical modeling for the social and behavioral sciences. New York: Plenum Press, 1-38.

Solga, H., Wagner, S., 2008. Die Zurückgelassenen - Die soziale Verarmung der Lernumwelt von Hauptschülern und Hauptschülerinnen. In: Becker, R., Lauterbach, W. (Hg.), Bildung als Privileg? Ursachen von Bildungsungleichheit aus soziologischer Sicht (3. A.). Wiesbaden: VS Verlag für Sozialwissenschaften, 189-217.

South, S.J., Messner, S.F., 2000. Crime and demography: multiple linkages, reciprocal relations. Annual Review of Sociology, 26, 83-106.

St. Jean, P.K., 2007. Pockets of crime: broken windows, collective efficacy, and the criminal point of view. Chicago: Chicago University Press.

Steenbeek, W., Hipp, J.R., 2011. A longitudinal test of social disorganization theory: feedback effects among cohesion, social control, and disorder. Criminology, 49(3), 833-870.

Stewart, E.A., Simons, R.L., 2010. Race, code of the street, and violent delinquency: a multilevel investigation of neighborhood street culture and individual norms of violence. Criminology, 48(2), 569-605.

Stolle, D., Soroka, S., Johnston, R., 2008. When does diversity erode trust? Neighborhood diversity, interpersonal trust and the mediating effect of social interactions. Political Studies, $56,57-75$.

Sutherland, E.H., Cressey, D.R., 1960. Principles of Criminology (6. A.). Chicago; Philadelphia; New York: J. B. Lippincott.

Sykes, B., Musterd, S., 2011. Examining neighbourhood and school effects simultaneously: what does the Dutch evidence show?. Urban Studies, 48(7), 1307-1331.

Taylor, R.B., 2001. Breaking away from broken windows: Baltimore neighborhoods and the nationwide fight against crime, grime, fear, and decline. Boulder, CO: Westview Press. 
Taylor, R.B., 2010. Communities, crime, and reactions to crime multilevel models: accomplishments and meta-challenges. Journal of Quantitative Criminology, 26, 455-466.

Taylor, R.B., Harris, P.W., Jones, P.R., Garcia, R.M., McCord, E.S., 2011. Ecological origins of shared perceptions of troublesome teen groups: implications for the basic systemic model of crime, the incivilities thesis, and political economy. Journal of Research in Crime and Delinquency, 48(2), 298-324.

Teasdale, B., Silver, E., 2009. Neighborhoods and self-control: toward an expanded view of socialization. Social Problems, 56(1), 205-222.

Thome, H., 2002. Kriminalität im Deutschen Kaiserreich, 1883-1914. Eine sozialökologische Analyse. Geschichte und Gesellschaft, 28(4), 519-553.

Thornberry, T.P., Krohn, M.D., Lizotte, A.J., Smith, C.A., Tobin, K., 2003. Gangs and delinquency in developmental perspective. Cambridge: Cambridge University Press.

Timberlake, J.M., 2007. Racial and ethnic inequality in the duration of children's exposure to neighborhood poverty and affluence. Social Problems, 54(3), 319-342.

Tita, G.E., Petras, T.L., Greenbaum, R.T., 2006. Crime and residential choice: a neighborhood level analysis of the impact of crime on housing prices. Journal of Quantitative Criminology, 22, 299-317.

van Ham, M., Manley, D., Bailey, N., Simpson, L., Maclennan, D. (Hg.), 2012. Neighbourhood effects research: new perspectives. Dordrecht; et al.: Springer.

Vazsonyi, A.T., Cleveland, H.H., Wiebe, R.P., 2006. Does the effect of impulsivity on delinquency vary by level of neighborhood disadvantage? Criminal Justice and Behavior, $33(4), 511-541$.

Verbitsky-Savitz, N., Raudenbush, S.W., 2009. Exploiting spatial dependence to improve measurement of neighborhood social processes. Sociological Methodology, 39(1), 151183.

Warner, B.D., Wilcox Rountree, P., 1997. Local social ties in a community and crime model: questioning the systemic nature of informal social control. Social Problems, 44, 520-536.

Warr, M., 2002. Companions in crime: the social aspects of criminal conduct. Cambridge: Cambridge University Press.

Weerman, F.M., 2011. Delinquent peers in context: a longitudinal network analysis of selection and influence effects. Criminology, 49(1), 253-286. 
Wheaton, B., Clarke, P., 2003. Space meets time: integrating temporal and contextual influences on mental health in early adulthood. American Sociological Review, 68(Oct), 680706.

Wiehe, S., Carroll, A., Liu, G., Haberkorn, K., Hoch, S., Wilson, J., Fortenberry, J.D., 2008. Using GPS-enabled cell phones to track the travel patterns of adolescents. International Journal of Health Geographics, 7(1), 22.

Wikström, P.-O.H., 2006. Individuals, settings, and acts of crime. situational mechanisms and the explanation of crime. In: Wikström, P.-O.H., Sampson, R.J. (Hg.), Crime and its explanation: contexts, mechanisms and development. Cambridge: Cambridge University Press, 61-107.

Wikström, P.-O.H., 2007. The social ecology of crime. The role of the environment in crime causation. In: Schneider H.J. (Hg.), Internationales Handbuch der Kriminologie, Bd. 1: Grundlagen der Kriminologie. Berlin: de Gruyter, 333-357.

Wikström, P.-O.H., 2010. Situational action theory. In: Cullen, F.T., Wilcox, P. (Hg.), Encyclopedia of criminological theory. Thousand Oaks, CA: Sage, 1000-1008.

Wikström, P.-O.H., Butterworth, D., 2006. Adolescent crime: individual differences and lifestyle risks. Cullompton: Willan.

Wikström, P.-O.H., Ceccato, V., Hardie, B., Treiber, K., 2010. Activity fields and the dynamics of crime. advancing knowledge about the role of the environment in crime causation. Journal of Quantitative Criminology, 26(1), 55-86.

Wikström, P.-O.H., Loeber, R., 2000. Do disadvantaged neighborhoods cause well-adjusted children to become adolescent delinquents? Criminology, 38, 1109-1142.

Wikström, P.-O.H., Oberwittler, D., Treiber, K., Hardie, B., 2012. Breaking rules. The social and situational dynamics of young people's urban crime. Oxford: Oxford University Press.

Wikström, P.-O.H., Sampson, R.J., 2003. Social mechanisms of community influences on crime and pathways in criminality. In: Lahey, B.B., Moffitt, T.E., Caspi, A. (Hg.), The causes of conduct disorder and serious juvenile delinquency. New York: Guilford Press, 118-148.

Wilson, J.Q., Kelling, G.E., 1982. Fixing broken windows: the police and neighborhood safety. Atlantic Monthly (March), 29-38. 
Wilson, W.J., 1987. The truly disadvantaged: the inner city, the underclass, and public policy. Chicago: Chicago University Press.

Wilson, W.J., 1997. When work disappears: the world of the new urban poor. New York:

Knopf.

Wodtke, G.T., Harding, D.J., Elwert, F., 2011. Neighborhood effects in temporal perspective: the impact of long-term exposure to concentrated disadvantage on high school graduation. American Sociological Review, 76(5), 713-736.

Wolf, C., Best, H., 2010. Lineare Regressionsanalyse. In: Wolf, C., Best, H. (Hg.), Handbuch der sozialwissenschaftlichen Datenanalyse. Wiesbaden: VS Verlag für Sozialwissenschaften, 607-638.

Wyant, B.R., 2008. Multilevel impacts of perceived incivilities and perceptions of crime risk on fear of crime isolating endogenous impacts. Journal of Research in Crime and Delinquency, 45(1), 39-64.

Xu, Y., Fiedler, M.L., Flaming, K.H., 2005. Discovering the impact of community policing: the broken windows thesis, collective efficacy, and citizen's judgement. Journal of Research in Crime and Delinquency, 42(2), 147-186.

Zahn, M.A., Browne, A., 2009. Gender differences in neighrborhood effects and delinquency. In: Zahn, M.A. (Hg.), The delinquent girl. Philadelphia: Temple University Press, 164181.

Zimmerman, G.M., Messner, S.F., 2010. Neighborhood context and the gender gap in adolescent violent crime. American Sociological Review, 75(6), 958-980. 


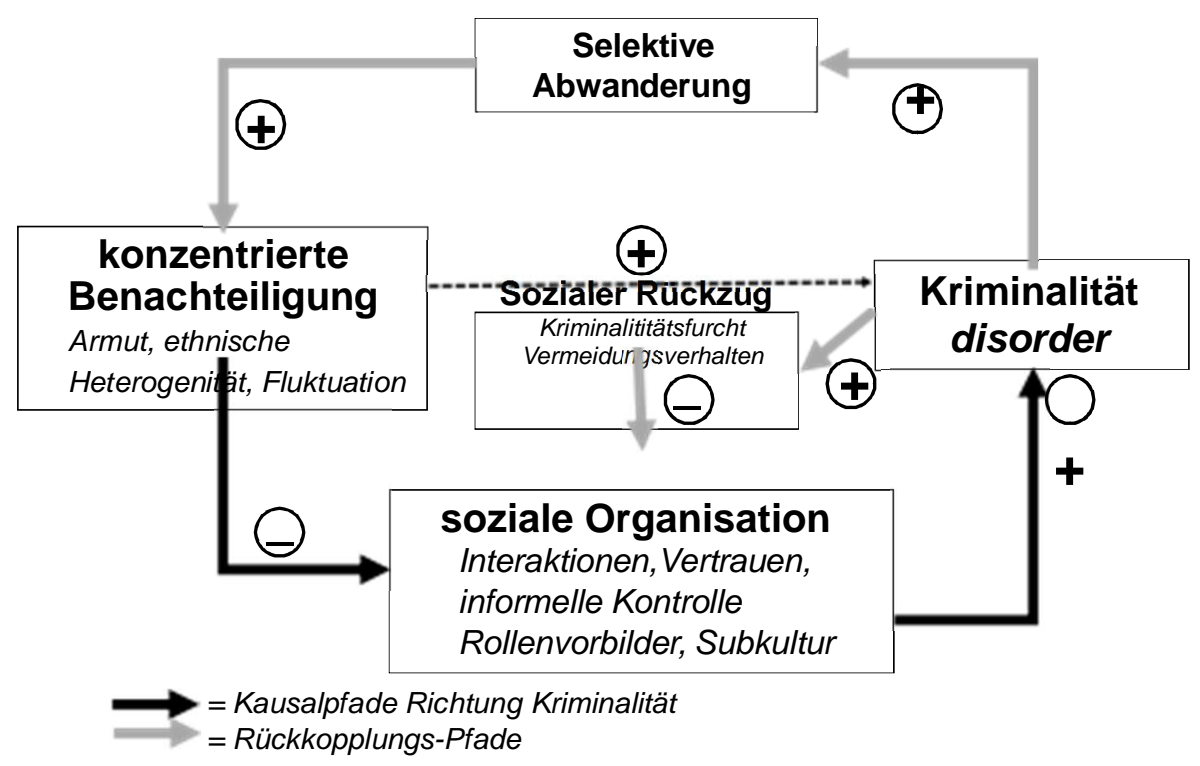

Abbildung 1: Das ,systemische Modell“ 


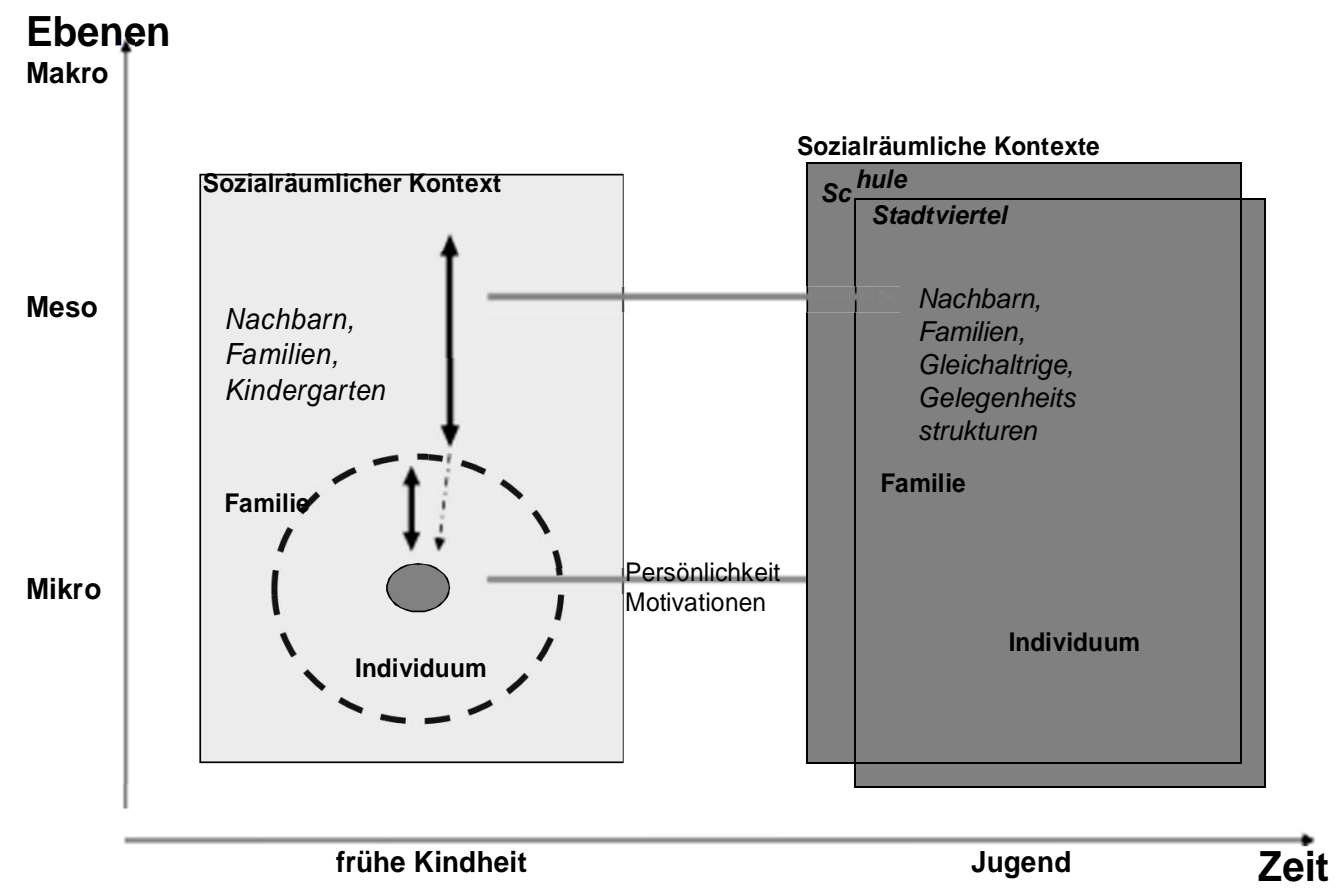

Abbildung 2: Theorie-Schema Kontexteffekte auf Kinder und Jugendliche 
Tab. 1: Übersicht über Studien zu sozialräumlichen Kontexteffekten auf Jugenddelinquenz

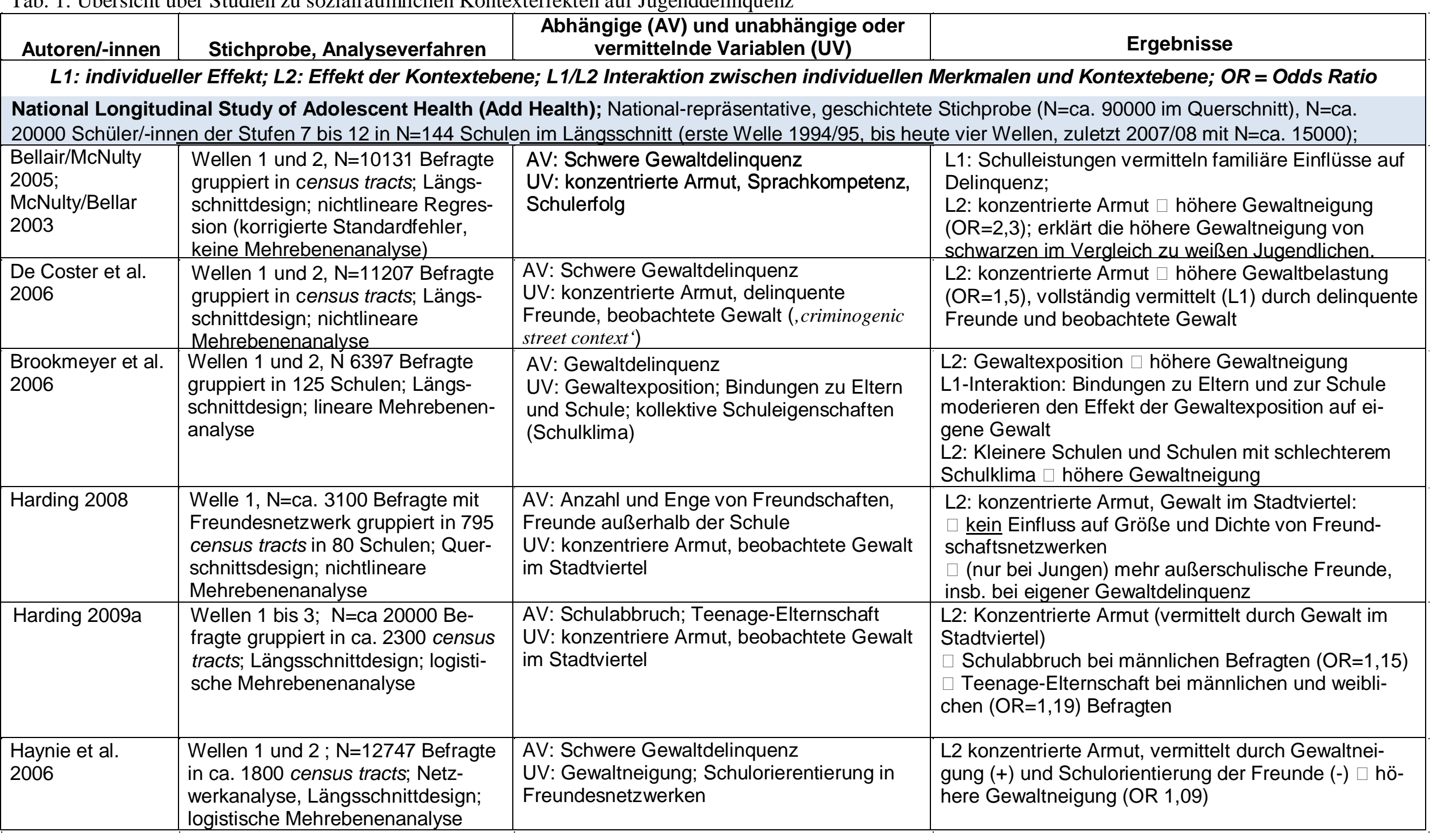




\begin{tabular}{|c|c|c|c|}
\hline Kreager 2007 & $\begin{array}{l}\text { Welle 1; N=51268 Befragte grup- } \\
\text { piert in } 129 \text { Schulen; Querschnitts- } \\
\text { design; nicht-lineare Mehrebenen- } \\
\text { analyse; für einige Analysen Wellen } \\
1 \text { und 2; } N=1100 \text { Befragte in einer } \\
\text { Schule; Längsschnittdesign }\end{array}$ & $\begin{array}{l}\text { AV: Popularität in Freundesnetzwerken } \\
\text { UV: kollektive Schulmerkmale (gemittelte Ge- } \\
\text { waltneigung der Schüler) }\end{array}$ & $\begin{array}{l}\text { Querschnittsanalyse: } \\
\text { L1: Gewaltneigung } \square \text { geringere Popularität } \\
\text { L1: Gewaltneigung } \square \text { höhere Popularität nur für Schü- } \\
\text { ler mit schlechten Leistungen } \\
\text { L1: Alkoholkonsum } \square \text { höhere Popularität Alkoholkon- } \\
\text { sum } \\
\text { L1/L2-Interaktion: Gewaltneigung bei Mädchen } \square \text { hö- } \\
\text { here Popularität nur in Schulen mit sehr hohem Ge- } \\
\text { waltniveau }\end{array}$ \\
\hline $\begin{array}{l}\text { Vazsonyi et al. } \\
2006\end{array}$ & $\begin{array}{l}\text { Wellen } 1 \text { und 2; } \mathrm{N}=19845 \text { Befragte } \\
\text { gruppiert in Schulen; lineare } \\
\text { Mehrebenenanalyse; } \\
\text { Aufteilung in Stadtviertel-Dezile } \\
\text { (nach konzentierter Armut), ANOVA }\end{array}$ & $\begin{array}{l}\text { AV: Gewaltdelinquenz; Impulsivität } \\
\text { UV: konzentrierte Armut }\end{array}$ & $\begin{array}{l}\text { L2: konzentrierte Armut } \square \text { weniger Impulsivität } \\
\text { L2: konzentrierte Armut } \square \text { höhere Gewaltneigung, } \\
\text { stärkerer Effekt bei Mädchen } \\
\text { L1/L2-Interaktion: nur bei Mädchen schwache Interak- } \\
\text { tion zwischen Impulsivität und konzentrierter Armut }\end{array}$ \\
\hline \multicolumn{4}{|c|}{$\begin{array}{l}\text { Project on Human Development in Chicago Neighborhoods (PHDCN); geschichtete Zufallsstichprobe (N=6228) von sieben Alterskohorten (0, 3, 6, 9, 12, 15, } \\
\text { und } 18 \text { Jahre), } 3 \text { Wellen }(1994-97,1997-99,2000-2001) \text { in } 80 \text { Stadtvierteln (neighborhood cluster) }\end{array}$} \\
\hline $\begin{array}{l}\text { Browning et al. } \\
2008\end{array}$ & $\begin{array}{l}\text { Wellen } 1 \text { und 2; Kohorten } 9 \text { und } 12 \\
\text { Jahre, N=768 Kinder gruppiert in } 80 \\
\text { Stadtvierteln, Längsschnittdesign, } \\
\text { nichtlineare Mehrebenenanalyse }\end{array}$ & $\begin{array}{l}\text { AV: Anzahl Sexualpartner } \\
\text { UV: konzentrierte Armut, kollektive Wirksam- } \\
\text { keit }\end{array}$ & $\begin{array}{l}\text { L2: kollektive Wirksamkeit } \square \text { geringe Wahrscheinlich- } \\
\text { keit multipler Sexualpartner }\end{array}$ \\
\hline $\begin{array}{l}\text { Maimon/ Brow- } \\
\text { ning } 2010\end{array}$ & $\begin{array}{l}\text { Wellen } 1 \text { bis } 3 \text {; Kohorten } 9 \text { und } 12 \\
\text { Jahr, } N=842 \text { Kinder gruppiert in } 80 \\
\text { Stadtvierteln, Längsschnittdesign: } \\
\text { nichtlineare Mehrebenenanalyse }\end{array}$ & $\begin{array}{l}\text { AV: ,action-orientierter' Freizeitstil; Gewaltde- } \\
\text { linquenz } \\
\text { UV: konzentrierte Armut, Migrantenanteil; } \\
\text { kollektive Wirksamkeit (L2); Familienbindung, } \\
\text { delinquente Peers (L1) }\end{array}$ & $\begin{array}{l}\text { L2: kollektive Wirksamkeit } \square \text { stärkerer, action-orien- } \\
\text { tierter' Freizeitstil } \\
\text { L2: Migrantenanteil } \square \text { niedrigere Gewaltneigung } \\
\text { L1/L2-Interaktion: kollektive Wirksamkeit moderiert } \\
\text { den Effekt des , action-orientierten` Freizeitstils auf Ge- walt } \\
\text { (stärkerer Effekt in Stadtvierteln mit niedriger kol- } \\
\text { lektiver Wirksamkeit) }\end{array}$ \\
\hline $\begin{array}{l}\text { Browning et al. } \\
2005\end{array}$ & $\begin{array}{l}\text { Wellen } 1 \text { und } 2 \text {; Kohorten } 12 \text { und } \\
15 \text { Jahre, N=907 Jugendliche grup- } \\
\text { piert in } 80 \text { Stadtvierteln, Längs- } \\
\text { schnittdesign; nichtlineare } \\
\text { Mehrebenenanalyse }\end{array}$ & $\begin{array}{l}\text { AV: Alter beim ersten Sexualkontakt } \\
\text { UV: konzentrierte Armut, Migrantenanteil; } \\
\text { kollektive Wirksamkeit (L2); Familienbindung, } \\
\text { Elternkontrolle (L1) }\end{array}$ & $\begin{array}{l}\text { L1/L2-Interaktion: kollektive Wirksamkeit } \square \text { höheres } \\
\text { Alter nur bei gleichzeitig niedriger Elternkontrolle }\end{array}$ \\
\hline $\begin{array}{l}\text { Fagan/Wright } \\
2012\end{array}$ & $\begin{array}{l}\text { Welle 1; Kohorten 9, 12, } 15 \text { Jahre, } \\
\mathrm{N}=2344 \text { Jugendliche gruppiert in } 80 \\
\text { Stadtvierteln; Querschnittsdesign; } \\
\text { nichtlineare Mehrebenenanalyse }\end{array}$ & $\begin{array}{l}\text { AV: (Gewalt-)delinquenz } \\
\text { UV: konzentrierte Armut, kollektive Wirksam- } \\
\text { keit }\end{array}$ & $\begin{array}{l}\text { L2: kollektive Wirksamkeit } \square \text { höhere Gewaltneigung } \\
\text { bei Mädchen, sonst keine Kontexteffekte (unter Kon- } \\
\text { trolle von delinquenten Freunden) }\end{array}$ \\
\hline $\begin{array}{l}\text { Bingenheimer et } \\
\text { al. } 2005\end{array}$ & $\begin{array}{l}\text { Wellen } 1 \text { bis } 3 \text {, Kohorten } 12 \text { und } 15 \\
\text { Jahre; } \mathrm{N}=1239 \text { (Welle } 2 \text { ) bzw. } 984\end{array}$ & $\begin{array}{l}\text { AV: schwere Gewaltdelinqenz } \\
\text { UV: beobachtete Gewalt mit Schusswaffen }\end{array}$ & $\begin{array}{l}\text { L1: Beobachtung von Gewalt mit Schusswaffen } \square \text { hö- } \\
\text { here Gewaltneigung (OR 2,4) }\end{array}$ \\
\hline
\end{tabular}




\begin{tabular}{|c|c|c|c|}
\hline & $\begin{array}{l}\text { Jugendliche (Welle } 3) \text { in } 78 \text { Stadt- } \\
\text { vierteln; Längsschnittdesign; pro- } \\
\text { pensity score matching mit } 37 \\
\text { Kovariaten }\end{array}$ & & \\
\hline Kirk 2008 & $\begin{array}{l}\text { Welle 1; Kohorten 12, 15, } 18 \text { Jahre, } \\
\mathrm{N}=1787 \text {, gruppiert in } 80 \text { Stadtvier- } \\
\text { teln; Wachstumskurven, Mehrebe- } \\
\text { nenanalyse }\end{array}$ & $\begin{array}{l}\text { AV: polizeiliche Registrierung } \\
\text { UV: Ethnie, konzentrierte Armut, kollektive } \\
\text { Wirksamkeit, Devianztoleranz }\end{array}$ & $\begin{array}{l}\text { L2: Stadtviertelkontext } \square \text { erklärt } 10 \% \text { der höheren } \\
\text { Wahrscheinlichkeit einer polizeilichen Registrierung } \\
\text { von Schwarzen vs. Weißen }\end{array}$ \\
\hline $\begin{array}{l}\text { Kirk/Matsuda } \\
2011\end{array}$ & $\begin{array}{l}\text { Wellen } 1 \text { bis 3, Kohorten } 15 \text { und } 18 \\
\text { Jahre; N=1071 (Welle 2) bzw. } 984 \\
\text { (Welle 3) gruppiert in } 78 \text { Stadtvier- } \\
\text { teln; Querschnittsdesign; nichtline- } \\
\text { are Mehrebenenanalyse }\end{array}$ & $\begin{array}{l}\text { AV: polizeiliche Registrierung } \\
\text { UV: Ethnie, konzentrierte Armut, kollektive } \\
\text { Wirksamkeit, legal cynicism }\end{array}$ & $\begin{array}{l}\text { L2: Anteil Schwarzer } \square \text { geringe Wahrscheinlichkeit } \\
\text { der polizeilichen Registrierung } \\
\text { L1/L2-Interaktion: legal cynicism (L2) moderiert die } \\
\text { Wahrscheinlichkeit der poliz. Registrierung für Delin. } \\
\text { quente (geringer in Stadtvierteln mit sehr hohem lega } \\
\text { cynicism) }\end{array}$ \\
\hline $\begin{array}{l}\text { Sampson et al. } \\
2005\end{array}$ & $\begin{array}{l}\text { Wellen } 1 \text { bis } 3, \text { Kohorten } 9,12,15 \text {, } \\
\text { und } 18 \text { Jahre; N=2955 gruppiert in } \\
180 \text { Stadtvierteln (census tracts); } \\
\text { Längschnittsdesign; nichtlineare } \\
\text { Mehrebenenanalyse }\end{array}$ & $\begin{array}{l}\text { AV: Gewaltdelinquenz } \\
\text { UV: Armut, soziale/ethnische Segregation, } \\
\text { frühere Gewaltkriminalität im Stadtviertel }\end{array}$ & $\begin{array}{l}\text { L2: Anteil höhere Berufe (-), frühere Gewaltkriminalität } \\
(+) \square \text { höhere Neigung zu Gewaltdelinquenz; Kontext } \\
\text { erklärt } 60 \% \text { der Höherbelastung von Jugendlichen } \\
\text { afro-amerikanischer vs. europäischer Abstammung }\end{array}$ \\
\hline $\begin{array}{l}\text { Sampson et al. } \\
2008\end{array}$ & $\begin{array}{l}\text { Wellen } 1 \text { bis 3; Kohorten 6, 9, } 12 \\
\text { Jahre, } \mathrm{N}=772 \text { Schwarze; Längs- } \\
\text { schnittdesign, propensity score } \\
\text { matching; Mehrebenenanalyse }\end{array}$ & $\begin{array}{l}\text { AV: Sprachkompetenz } \\
\text { UV: konzentrierte Armut }\end{array}$ & $\begin{array}{l}\text { L2: konzentrierte Armut } \square 0,25 \text { SD geringe Sprach- } \\
\text { kompetenz im Zeitverlauf (und kumulativ) }\end{array}$ \\
\hline \multicolumn{4}{|c|}{$\begin{array}{l}\text { Moving to Opportunity (MTO); randomisiertes Experiment (Umzug in nicht-arme Stadtviertel) in vier US-Städten; 1994-97, durchschnittl. 5,7 Jahre follow-up; } \\
\text { N=1807 Jugendiche }\end{array}$} \\
\hline Kling et al. 2005 & $\begin{array}{l}\mathrm{N}=1807 \text { Jugendliche, experimentel- } \\
\text { les Längsschnittdesign }\end{array}$ & AV: Delinqenz (poliz. Registrierungen) & $\begin{array}{l}\text { L2: Umzug } \square \text { für Mädchen ca. 30\% Rückgang der } \\
\text { Delinquenz); für Jungen kein Rückgang bei Gewaltde- } \\
\text { linquenz, ca. } 30 \% \text { Anstieg Eigentumsdelinquenz }\end{array}$ \\
\hline $\begin{array}{l}\text { Clampet-Lundqu- } \\
\text { ist et al.2011 }\end{array}$ & $\begin{array}{l}\mathrm{N}=34 \text { Umzugs-Gruppe, } \mathrm{N}=52 \text { Ver- } \\
\text { gleichsgruppe, halb-standardisierte, } \\
\text { qualitative Interviews }\end{array}$ & $\begin{array}{l}\text { Entwicklung der allgemeinen Lebensituatio } \\
\text { und des delinquenten Verhaltens nach den } \\
\text { Umzug aus einem sehr armen Wohnquartier } \\
\text { ein Mittelschichtsquartier; Freizeitverhalten } \\
\text { und Beziehung zu Gleichaltrigen }\end{array}$ & $\begin{array}{l}\text { nMädchen profitieren stärker von dem Umzug, weil sie } \\
\text { nweniger exponiert (in der Öffentlichkeit) leben als Jun- } \\
\text { ngen, und Jungen spezielle Fähigkeiten brauchen, si- } \\
\text { cher durch sozialräumlich verortete Risiken zu navi- } \\
\text { gieren. Aufgrund ihrer zuvor erworbenen Verhaltenso- } \\
\text { rientierungen fallen sie im Mittelschichtsquartier stär- } \\
\text { ker als abweichend auf. }\end{array}$ \\
\hline \multicolumn{4}{|c|}{ Weitere U.S.-amerikanische Studien } \\
\hline $\begin{array}{l}\text { Rankin/Quane } \\
2002\end{array}$ & $\begin{array}{l}\text { „Youth Achievement and the Struc- } \\
\text { ture of Inner City Communities”- }\end{array}$ & AV: prosoziales Verhalten; Problemverhalten & $\begin{array}{l}\text { L2/L1-Interaktion: Kollektive Wirksamkeit } \square \text { nur wenn } \\
\text { elterliche Kontrolle schwach ist, geringe Neigung zu }\end{array}$ \\
\hline
\end{tabular}




\begin{tabular}{|c|c|c|c|}
\hline & $\begin{array}{l}\text { Studie, Chicago,N= } 636 \text { afro-ameri- } \\
\text { kanische Jugendliche und deren } \\
\text { Eltern, gruppiert in } 59 \text { census tracts } \\
\text { mit hoher soz. Benachteiligung; } \\
\text { Querschnittsdesign; lineare } \\
\text { Mehrebenenanalyse }\end{array}$ & $\begin{array}{l}\text { UV: konzentrierte Armut, kollektive Wirksam- } \\
\text { keit, elterliche Kontrolle }\end{array}$ & $\begin{array}{l}\text { Problemverhalten; bei starker elterlicher Kontrolle kein } \\
\text { Effekt des Stadtviertelkontextes }\end{array}$ \\
\hline $\begin{array}{l}\text { Stewart/Simons } \\
2010\end{array}$ & $\begin{array}{l}\text { "Family and Community Health } \\
\text { Study", N=763 afro-amerikanische } \\
\text { Jugendliche, } 12 \text { bis } 15 \text { Jahre, grup- } \\
\text { piert in } 71 \text { census tracts in Georgia } \\
\text { und lowa, } 2 \text { Wellen; Längsschnitt- } \\
\text { design; lineare Mehrebenenanalyse }\end{array}$ & $\begin{array}{l}\text { AV: Gewaltdelinquenz } \\
\text { UV. Konzentrierte Armut, Homizidrate, ,street } \\
\text { culture“, }\end{array}$ & $\begin{array}{l}\text { L2: Konzentrierte Armut, Homizidrate und „,street cul- } \\
\text { ture“ } \square \text { höhere Neigung zu Gewaltdelinquenz } \\
\text { L1/L2-Interaktion: „street culture“ im Kontext verstärkt } \\
\text { den Effekt der individuellen Zustimmung zur street } \\
\text { culture auf Gewalt }\end{array}$ \\
\hline \multicolumn{4}{|c|}{ Europäische Studien } \\
\hline Helbig 2010 & $\begin{array}{l}\text { ELEMENT-Studie Berlin, N=3169 } \\
\text { Kinder gruppiert in } \mathrm{N}=71 \text { Grund- } \\
\text { schulen (gleichgesetzt mit Ver- } \\
\text { kehrszellen/Stadtvierteln), } 2 \text { Wellen } \\
\text { (4., 6. Stufe), Längsschnittdesign, } \\
\text { lineare Mehrebenenanalyse, }\end{array}$ & $\begin{array}{l}\text { AV: Sprach-, Mathematikkompetenz } \\
\text { V: konzentrierte Armut, Migrantenanteil }\end{array}$ & $\begin{array}{l}\text { L2: Abwesenheit von konzentrierter Armut } \square \text { höhere } \\
\text { Sprach-/Mathekompetenz (nichtlinearer Effekt, am } \\
\text { stärksten in Kontexten mit geringster Konzentration) } \\
\text { L2 Migrantenanteil } \square \text { schwacher Effekt auf Sprach-, } \\
\text { kein Effekt auf Mathekompetenz }\end{array}$ \\
\hline $\begin{array}{l}\text { Bernburg/ Thor- } \\
\text { lindsson } 2007\end{array}$ & $\begin{array}{l}\text { Island (Vollerhebung), N=6458 Ju- } \\
\text { gendliche } 15-16 \text { Jahre, gruppiert in } \\
\mathrm{N}=68 \text { Schulen (gleichgesetzt mit } \\
\text { Stadtvierteln/Gemeinden), Quer- } \\
\text { schnittsdesign, Mehrebenenana- } \\
\text { lyse }\end{array}$ & $\begin{array}{l}\text { AV: Delinqenz } \\
\text { V: Instabilität des Stadtviertels (Anteil unvoll- } \\
\text { ständiger Familien, residentielle Mobilität) }\end{array}$ & $\begin{array}{l}\text { L2: Instabilität } \square \text { höhere Delinquenzneigung } \\
\text { L1/L2-Interaktion: Instabilität } \square \text { verstärkt den Effekt } \\
\text { von , action'-orientierter Freizeitstil auf Delinquenz }\end{array}$ \\
\hline Brännström 2008 & $\begin{array}{l}\text { Schweden, } 26384 \text { Jugendliche } \\
\text { gruppiert in } 389 \text { Schulen und } 2309 \\
\text { Stadtvierteln; Querschnittsdesign, } \\
\text { kreuzklassifizierte lineare Mehrebe- } \\
\text { nenmodelle, }\end{array}$ & $\begin{array}{l}\text { AV: Schulleistungen } \\
\text { UV: konzentrierte Armut; ethnische Segrega- } \\
\text { tion }\end{array}$ & $\begin{array}{l}\text { L2: Schuleffekte wesentlich stärker als Stadtviertelef- } \\
\text { fekte; konzentrierte Armut } \square \text { schlechtere Schulleistun- } \\
\text { gen } \\
\text { L1/L2-Interaktionen: } \\
\text { individueller Migrationshintergrund in Schulen mit ho- } \\
\text { hem Migrantenanteil } \square \text { höhere Schulleistungen } \\
\text { individueller Migrationshintergrund in Stadtvierteln mit } \\
\text { konzentrierter Armut } \square \text { niedrigere Schulleistungen }\end{array}$ \\
\hline Pauwels 2007 & $\begin{array}{l}\mathrm{N}=2485 \text { Jugendliche gruppiert in } \\
\mathrm{N}=42 \text { Stadtvierteln; Querschnittsde- } \\
\text { sign, lineare Mehrebenenanalyse }\end{array}$ & $\begin{array}{l}\text { AV: Delinquenz } \\
\text { UV: konzentrierte Armut }\end{array}$ & $\begin{array}{l}\text { L2 konzentrierte Armut } \square \text { keine höhere Delinquenz- } \\
\text { neigung }\end{array}$ \\
\hline
\end{tabular}




\begin{tabular}{|c|c|c|c|}
\hline $\begin{array}{l}\text { Wikström/ Butter- } \\
\text { worth } 2006\end{array}$ & $\begin{array}{l}\mathrm{N}=1957 \text { Jugendliche gruppiert in } \\
\mathrm{N}=286 \text { Stadtvierteln (enumeration } \\
\text { districts); Querschnittsdesign; OLS } \\
\text { und logistische Regression }\end{array}$ & $\begin{array}{l}\text { AV: Delinquenz } \\
\text { UV: konzentrierte Armut }\end{array}$ & $\begin{array}{l}\text { L2: konzentrierte Armut } \square \text { keine höhere Delinquenz- } \\
\text { neigung insgesamt, aber höhere Delinquenz im eige- } \\
\text { nen Wohngebiet }\end{array}$ \\
\hline $\begin{array}{l}\text { Reijneveld et al. } \\
2005\end{array}$ & $\begin{array}{l}\text { Niederlande, } \mathrm{N}=4480 \text { Kinder }(4-16 \\
\text { Jahre), Elternbericht und professio- } \\
\text { nelle Diagnostik; Querschnittsde- } \\
\text { sign; logistische kreuzklassifizierte } \\
\text { Mehrebenenmodelle }\end{array}$ & $\begin{array}{l}\text { AV: externalisierende Verhaltensprobleme } \\
\text { UV: konzentrierte Armut }\end{array}$ & $\begin{array}{l}\text { L2: konzentrierte Armut } \square \text { höhere Neigung zu Verhal- } \\
\text { tensproblemen (OR 1,69) }\end{array}$ \\
\hline Oberwittler 2004b & $\begin{array}{l}\text { Köln, Freiburg, } N=1586 \text { deutsche } \\
\text { Jugendliche (13-16 Jahre) mit loka- } \\
\text { len Freunden in } N=55 \text { Stadtvierteln, } \\
\text { Querschnittsdesign; nichtlineare } \\
\text { Mehrebenenmodelle }\end{array}$ & $\begin{array}{l}\text { AV: schwere Delinquenz } \\
\text { UV: konzentrierte Armut }\end{array}$ & $\begin{array}{l}\text { L2: konzentrierte Armut } \square \text { höhere Delinquenzneigung } \\
\text { (OR=1,98 für deutsche Jugendliche mit lokalem } \\
\text { Freundeskreis), kein Effekt für Jugendliche mit Migra- } \\
\text { tionshintergrund und Jugendliche ohne lokalen Freun- } \\
\text { deskreis }\end{array}$ \\
\hline Oberwittler 2007b & $\begin{array}{l}\text { Köln, Freiburg, } \mathrm{N}=2466 \text { weibliche } \\
\text { Jugendliche (13-16 Jahre) in } \mathrm{N}=61 \\
\text { Stadtvierteln, Querschnittsdesign; } \\
\text { nichtlineare Mehrebenenmodelle }\end{array}$ & $\begin{array}{l}\text { AV: Mitgliedschaft in gewaltorientierter Clique } \\
\text { UV: konzentrierte Armut, Migrationsstatus } \\
\text { (L1) }\end{array}$ & $\begin{array}{l}\text { L1/L2 Interaktion: konzentrierte Armut } \square \text { höhere } \\
\text { Wahrscheinlichkeit der Mitgliedschaft für einheimische } \\
\text { Mädchen, } \square \text { niedrigere Wahrscheinlichkeit für Mäd- } \\
\text { chen mit Migrationshintergrund }\end{array}$ \\
\hline
\end{tabular}

